\title{
Faster Algorithms for Computing the Stationary Distribution, Simulating Random Walks, and More
}

\author{
Michael B. Cohen* \\ MIT \\ micohen@mit.edu
}

\author{
Jonathan Kelner* \\ MIT \\ kelner@mit.edu
}

Aaron Sidford

Stanford University

sidford@stanford.edu

\author{
John Peebles ${ }^{+}$ \\ MIT \\ jpeebles@mit.edu
}

Adrian Vladu*

MIT

avladu@mit.edu

\begin{abstract}
In this paper, we provide faster algorithms for computing various fundamental quantities associated with random walks on a directed graph, including the stationary distribution, personalized PageRank vectors, hitting times, and escape probabilities. In particular, on a directed graph with $n$ vertices and $m$ edges, we show how to compute each quantity in time $\tilde{O}\left(m^{3 / 4} n+m n^{2 / 3}\right)$, where the $\tilde{O}$ notation suppresses polylogarithmic factors in $n$, the desired accuracy, and the appropriate condition number (i.e. the mixing time or restart probability).

Our result improves upon the previous fastest running times for these problems; previous results either invoke a general purpose linear system solver on a $n \times n$ matrix with $m$ non-zero entries, or depend polynomially on the desired error or natural condition number associated with the problem (i.e. the mixing time or restart probability). For sparse graphs, we obtain a running time of $\tilde{O}\left(n^{7 / 4}\right)$, breaking the $O\left(n^{2}\right)$ barrier of the best running time one could hope to achieve using fast matrix multiplication.

We achieve our result by providing a similar running time improvement for solving directed Laplacian systems, a natural directed or asymmetric analog of the well studied symmetric or undirected Laplacian systems. We show how to solve such systems in time $\tilde{O}\left(m^{3 / 4} n+m n^{2 / 3}\right)$, and efficiently reduce a broad range of problems to solving $\tilde{O}(1)$ directed Laplacian systems on Eulerian graphs. We hope these results and our analysis open the door for further study into directed spectral graph theory.
\end{abstract}

\footnotetext{
${ }^{*}$ This material is based upon work supported by the National Science Foundation under Grant No. 1111109.

+ This material is based upon work supported by the National Science Foundation Graduate Research Fellowship under Grant No. 1122374 and by the National Science Foundation under Grant No. 1065125.

${ }^{\ddagger}$ This material is partly based upon work supported by the National Science Foundation under Grant No. 1637566. Part of this work was done while at MIT.
} 


\section{Introduction}

The application and development of spectral graph theory has been one of the great algorithmic success stories of the past three decades. By exploiting the relationship between the combinatorial properties of a graph, the linear algebraic properties of its Laplacian, and the probabilistic behavior of the random walks they induce, researchers have obtained landmark results ranging across multiple areas in the theory of algorithms, including Markov chain Monte Carlo techniques for counting [50, 21, 19] and volume estimation [38, 13, 59, 39, 40, 35], approximation algorithms for clustering and graph partitioning problems [2, 52, 20, 5, 43], derandomization [17, 48], error correcting codes [53, 51], and the analysis of random processes [37], among others. In addition to their theoretical impact, spectral techniques have found broad applications in practice, forming the core of Google's PageRank algorithm, playing a ubiquitous role in practical codes for machine learning, computer vision, clustering, and graph visualization. Furthermore they have enabled the computation of fundamental properties of various Markov chains, such as stationary distributions, escape probabilities, hitting times, mixing times, and commute times.

More recently, spectral graph theory has been driving an emerging confluence of algorithmic graph theory, numerical scientific computing, and convex optimization. This recent line of work began with a sequence of papers that used combinatorial techniques to accelerate the solution of linear systems in undirected graph Laplacians, eventually leading to algorithms that solve these systems in nearly-linear time $[52,26,27,28,25,33,10,47,31,29]$. This was followed by an array of papers in the so-called "Laplacian Paradigm" [57], which either used this nearly-lineartime algorithm as a primitive or built on the structural properties underlying it to obtain faster algorithms for problems at the core of algorithmic graph theory, including finding maximum flows and minimum cuts [7, 32, 49, 22, 46], solving traveling salesman problems [6, 3], sampling random trees [23, 42], sparsifying graphs [54, 55, 1, 30], computing multicommodity flows [24, 22], and approximately solving a wide range of general clustering and partitioning problems [2, 20, 5, 43].

While these recent algorithmic approaches have been very successful at obtaining algorithms running in close to linear time for undirected graphs, the directed case has conspicuously lagged its undirected counterpart. With a small number of exceptions involving graphs with particularly nice properties and a line of research in using Laplacian system solvers inside interior point methods for linear programming $[41,34,11]$, the results in this line of research have centered almost entirely on the spectral theory of undirected graphs. While there have been interesting results in candidate directed spectral graph theory $[8,5,15]$, their algorithmic ramifications have been less clear.

One problem that particularly well illustrates the discrepancy between the directed and undirected settings is the computation of the stationary distribution of a random walk. Computing this is a primary goal in the analysis of Markov chains, constitutes the main step in the PageRank algorithm, remains the missing piece in derandomizing randomized log space computations [9], and is necessary to obtain the appropriate normalization for any of the theoretical or algorithmic results in one of the few instantiations of directed spectral graph theory $[8,5]$.

In the undirected setting, the stationary distribution is proportional to the degree of a vertex, so it can be computed trivially. However, despite extensive study in the mathematics, computer science, operations research, and numerical scientific computing communities, the best previously known asymptotic guarantees for this problem are essentially what one gets by applying generalpurpose linear algebra routines. Given a directed graph with $n$ vertices and $m$ edges these previous algorithms fall into two broad classes:

- Iterative Methods: These aim to compute the stationary distribution by either simulating the random walk directly or casting it as a linear system or eigenvector computation and 
applying either a global or coordinate-wise iterative method to find it. The running times of these methods either depend polynomially on the relevant numerical conditioning property of the instance, which in this case is, up to polynomial factors, the mixing time of the random process; or they only compute a distribution that only approximately satisfies the defining equations of the stationary distribution, with a running time that is polynomial in $1 / \epsilon$. There has been extensive work on tuning and specializing these methods to efficiently compute the stationary distribution, particularly in the special case of PageRank. However, all such methods that we are aware of retain a polynomial dependence on either the mixing time, which can be arbitrary large as a function of the number of edges of the graph, or on $1 / \epsilon{ }^{1}$

- Fast Matrix Multiplication: By using a direct method based on fast matrix multiplication, one can find the stationary distribution in time in time $n^{\omega}$, where $\omega<2.3729$ [60] is the matrix multiplication exponent. These methods neglect the graph structure and cannot exploit sparsity. As such, even if one found a matrix multiplication algorithm matching the lower bound of $\omega=2$, this cannot give a running time lower than $\Omega\left(n^{2}\right)$, even when the graph is sparse.

Another problem which well demonstrates the gap between directed and undirected graph problems is that of solving linear systems involving graph Laplacians. For undirected graphs, as we have discussed there are multiple algorithms to solve associated Laplacian systems in nearly time. However, in the case of directed graphs natural extensions of solving Laplacian systems are closely related to computing the stationary distribution, and thus all known algorithms either depend polynomially on the condition number of the matrix or the desired accuracy or they require time $\Omega\left(n^{2}\right)$. Moreover, many of the techniques, constructions, and properties used to solve undirected Laplacian systems either have no known analogues for directed graphs or can be explicitly shown to not exist. This gap in our ability to solve Laplacian systems is one of the the primary reasons (perhaps the primary reason) that the recent wave of graph algorithms based on the "Laplacian Paradigm" have not produced directed results to match the undirected ones.

Given the fact that, despite several decades of work on designing specialized methods for this problem, there are no methods known that asymptotically improve upon general linear algebra routines, along with the structural problems in translating the techniques from the undirected case, it would not be unreasonable to expect that the best one can hope for is heuristic improvements in special cases, and that the worst-case asymptotics for graph Laplacians are no better than the $\min O\left(n^{\omega}, n m\right) \geq \Omega\left(n^{2}\right)$ that is known for general matrices.

In this paper, we show that this is not the case by providing an algorithm that solves directed graph Laplacian systems - a natural generalization of undirected graph Laplacian systems - in time $\tilde{O}\left(n m^{3 / 4}+n^{2 / 3} m\right)$ where here and throughout the paper the $\tilde{O}(\cdot)$ notation hides polylogarithmic factors in $n$, the desired accuracy, and the natural condition numbers associated with the problem. Consequently, we obtain the first asymptotic improvement for these systems over solving general linear systems. ${ }^{2}$ In particular, when the graph is sparse, i.e. $m=O(n)$, our algorithm runs in time $\tilde{O}\left(n^{7 / 4}\right)$, breaking the barrier of $O\left(n^{2}\right)$ that would be achieved by algorithms based on fast matrix multiplication if $\omega=2$. We then leverage this result to obtain improved running times for a host of problems in algorithmic graph theory, scientific computing, and numerical linear algebra, including:

\footnotetext{
${ }^{1} \mathrm{~A}$ possibleexception, is the algorithm that invokes conjugate gradient in a blackbox manner to solve the requisite linear system to compute the stationary distribution. At best this analysis would suggest an $O(m n)$ running time. However, it is not known how to realize even this running time in the standard word-RAM model of computation.

${ }^{2}$ In follow up work, the authors of this paper in collaboration with Anup Rao have improved the running time to almost linear in the number of edges in the graph, meaning the running time is linear if we ignore contributions to the running time that are smaller than any polynomial. This paper will be made available online as soon as possible.
} 
- Computing the Stationary Distribution: We compute a vector within $\ell_{2}$ distance $\epsilon$ of the stationary distribution of a random walk on a strongly connected directed graph in time $\tilde{O}\left(n m^{3 / 4}+n^{2 / 3} m\right)$, where the natural condition number of this problem is the mixing time. (See Section 7.2.)

- Solving Large Classes of Linear Systems: We provide algorithms that solve a large class of well-studied linear systems. Compared with prior algorithms capable of solving this class, ours are the first that are asymptotically faster than solving general linear systems, and the first that break the $O\left(n^{2}\right)$ barrier for sufficiently sparse instances. Our methods solve directed Laplacian systems and systems where the matrix is row- or column-diagonally dominant. The running time is $\tilde{O}\left(n m^{3 / 4}+n^{2 / 3} m\right)$. (See Section 7.3.)

- Computing Personalized PageRank: We compute a vector within $\ell_{2}$ distance $\epsilon$ of the personalized PageRank vector, for a directed graph with with restart probability $\beta$, in time $\tilde{O}\left(n m^{3 / 4}+n^{2 / 3} m\right)$. Here the natural condition number is $1 / \beta$. In the case of small $\beta$ and $\epsilon$, this improves upon local methods that take $O\left(m \beta^{-1} \epsilon^{-1}\right)$ time $[44,18,14,5,4,36]$. (See Section 7.1).

- Simulating Random Walks: We show how to compute a wide range of properties of random walks on directed graphs including escape probabilities, commute times, and hitting times. (See Section 7.4 and Section 7.5.) We also show how to efficiently estimate the mixing time of a lazy random walk on a directed graph up to polynomial factors in $n$ and the mixing time. (See Section 7.2.) The runtime for all these algorithms is $\tilde{O}\left(n m^{3 / 4}+n^{2 / 3} m\right)$.

- Estimating All Commute Times: We show how to build a $\tilde{O}\left(n \epsilon^{-2} \log n\right)$ size data structure in time $\tilde{O}\left(n m^{3 / 4}+n^{2 / 3} m\right)$ that, when queried with any two vertices $a$ and $b$, outputs a $1 \pm \epsilon$ multiplicative approximation to the expected commute time between $a$ and $b$, i.e. the expected amount of time for a random walk starting at $a$ to reach $b$ and return to $a$. Our data structure is similar to the data structure known for computing all-pairs effective resistances in undirected graphs [54, 55]. (See Section 7.6.)

It is important to note that the $\tilde{O}$-notation hides factors that are polylogarithmic in both the condition number (equivalently, mixing time) and the ratio of maximum to minimum stationary probability. As such, the natural parameter regime for our algorithms is when these quantities are subexponential or polynomial. For all the above problems, the best prior algorithms had worst case runtimes no better than $O\left(\min \left\{n^{\omega}, n m\right\}\right) \geq \Omega\left(n^{2}\right)$ in this regime. We hope that our results open the door for further research into directed spectral graph theory, and serve as foundation for the development of faster algorithms for directed graphs.

\section{$1.1 \quad$ Approach}

Our approach for solving these problems centers around solving linear systems in a class of matrices we refer to as directed (graph) Laplacians, a natural generalization of undirected graph Laplacians. A directed Laplacian, $\mathcal{L} \in \mathcal{R}^{n \times n}$, is simply a matrix with non-positive off-diagonal entries such that each diagonal entry is equal to the sum of the absolute value of the other off-diagonal entries in that column, i.e. $\mathcal{L}_{i j} \leq 0$ for $i \neq j$ and $\mathcal{L}_{i i}=-\sum_{j \neq i} \mathcal{L}_{j i}$ (equivalently $\overrightarrow{1}^{\top} \mathcal{L}=\overrightarrow{0}$ ). As with undirected Laplacians, every directed Laplacian there is naturally associated with a directed graph $G=(V, E, w)$, where the vertices $V$ correspond to the columns of $\mathcal{L}$ and there is an edge from vertex $i$ to vertex $j$ of weight $\alpha$ if and only if $\mathcal{L}_{j i}=-\alpha$. 
Another close feature of directed and undirected Laplacians is the close connection between random walks on the associated graph $G$ and solutions to linear systems in $\mathcal{L}$. We ultimately show that solving a small number of directed Laplacian systems suffices to obtain all of our desired applications (See Section 5 and Section 7). Unfortunately, solving linear systems in $\mathcal{L}$ directly is quite challenging. Particularly troubling is the fact that we while we know $\mathcal{L}$ has a non-trivial kernel (since $\overrightarrow{1}^{\top} \mathcal{L}=\overrightarrow{0}^{\top}$ ), we do not have a simple method to compute it efficiently. Moreover, $\mathcal{L}$ is not symmetric, complicating the analysis of standard iterative algorithms.Furthermore, the standard approach of multiplying on the left by the transpose, so that we are solving linear systems in $\mathcal{L}^{\top} \mathcal{L}$, would destroy the combinatorial structure of the problem and cause an intolerably large condition number. A natural idea is to try to work with a symmetrization of this matrix, $\frac{1}{2}\left(\mathcal{L}+\mathcal{L}^{\top}\right)$, but it turns out that this may not even be positive semidefinite (PSD). ${ }^{3}$ Consequently, it is not clear a priori how to define an efficient iterative method for computing the stationary $\mathcal{L}$ or solve systems in it without depending polynomially on the condition number of $\mathcal{L}$.

Fortunately, we do know how to characterize the kernel of $\mathcal{L}$, even if computing it is difficult $a$ priori. If we let $\mathbf{D} \in \mathcal{R}^{n \times n}$ denote the diagonal matrix consistent with $\mathcal{L}$, i.e., $\mathbf{D}_{i i}=\mathcal{L}_{i i}$, then we see that $\mathcal{L} \mathbf{D}^{-1}=\mathbf{I}-\mathbf{W}$ where $\mathbf{I}$ is the identity matrix and $\mathbf{W}$ is the random walk matrix associated with $G$. In other words, for any distribution $p$, we have that $\mathbf{W} p$ is the resulting distribution of one step of the random walk where, at a vertex $i \in[n]$, we pick a random outgoing edge with probability proportional to its weight and follow that edge. The Perron-Frobenius Theorem implies that as long as the graph is strongly connected there is some stationary distribution $s \in \mathcal{R}_{>0}$ such that $\mathbf{W} s=s$. Consequently, the kernel of $\mathcal{L}$ is simply the stationary distribution of the natural random walk on $G$ multiplied by $\mathbf{D}$.

Consequently, we can show that for every directed Laplacian $\mathcal{L}$ that corresponds to a strongly connected graph, there is always a vector $x \in \mathcal{R}_{>0}^{n}$ such that $\mathcal{L} x=0$ (See Lemma 1). In other words, letting $\mathbf{X}$ denote the diagonal matrix associated with $x$ the directed Laplacian $\mathcal{L}^{\prime}=\mathcal{L} \mathbf{X}$ satisfies $\mathcal{L}^{\prime} \mathbf{X} 1=0$. This says that the total weight of incoming edges to a vertex is the same as the total weight of outgoing edges from that vertex, i.e., that $\mathcal{L}^{\prime}$ corresponds to the Laplacian of an Eulerian graph. We call such a vector an Eulerian scaling of $\mathcal{L}$.

Now, solving systems in an Eulerian Laplacian $\mathcal{L}$ (i.e., a Laplacian corresponding to an Eulerian graph) seems easier than solving an arbitrary directed Laplacian. In particular, we know the kernel of a $\mathcal{L}$, since it is just the all ones vector. In addition, we have that $\frac{1}{2}\left(\mathcal{L}+\mathcal{L}^{\top}\right)$ is symmetric and PSD - in fact it is just the Laplacian of an undirected graph! Unfortunately, this does not immediately yield an algorithm, as it is not known how to use the ability to solve systems in such a symmetrization to solve systems in the original matrix.

Ultimately, this line of reasoning leaves us with two fundamental questions:

1. Can we solve Eulerian Laplacian systems in time $o\left(n^{\omega}, n m\right)$ ?

2. Can we use an Eulerian Laplacian system solver for more than solving Eulerian Laplacian systems?

The major contribution of this paper is answering both of these questions in the affirmative. We show the following:

- We show that we can solve Eulerian Laplacian systems in time $\tilde{O}\left(n m^{3 / 4}+n^{2 / 3} m\right)$.

\footnotetext{
${ }^{3}$ Consider the directed edge Laplacian $\mathcal{L}=\left[\begin{array}{cc}1 & 0 \\ -1 & 0\end{array}\right]$. Then, $\mathcal{L}+\mathcal{L}^{+}=\left[\begin{array}{cc}2 & -1 \\ -1 & 0\end{array}\right]$ has an eigenvector $(\sqrt{2}-1,1)$ with a corresponding eigenvalue of $(1-\sqrt{2})$.
} 
- We show that using Eulerian Laplacian systems we can solve broader classes of matrices we refer to as RCDD Z-matrices, and $\alpha$ RCDD Z-matrices.

- We show that using solvers for $\alpha$ RCDD Z-matrices, we can estimate an Eulerian scaling of a directed Laplacian.

- Putting these components together we achieve our desired applications. Some of these are applications are straightforward, whereas others require some significant work.

A serious question that arises throughout these results is the degree of precision do we need to carry out our arithmetic operations. This arrises both in using undirected Laplacian system solvers to solving Eulerian Laplacian systems, and then again in using Eulerian Laplacian system solvers to derive the rest of our results. These numerical issues are not merely technicalities - they crucially affect the algorithms we can use to solve our problem. In fact, we will see in Section 4 that, if we disregarded the numerics and relied on frequently-quoted assertions relating the behavior of conjugate gradient to the existence of polynomials with certain properties, we would actually obtain a better running time, but that these assertions do not carry over to reasonable finite-precision setting.

Given these subtleties, we discuss numerical issues throughout the paper, and in Appendix A we cover particular details of the stability of our algorithms and the precision they require, showing that we can achieve all our results in the standard unit cost RAM model (or any other reasonable model of computation).

In the remainder of this overview we briefly comment on the key technical ingredients of each of these results.

\subsubsection{Solving Eulerian Laplacian Systems}

To solve a Eulerian Laplacian system $\mathcal{L} x=b$, we first precondition, multiplying both sides by $\mathcal{L}^{\top} \mathbf{U}^{+}$, where $\mathbf{U} \stackrel{\text { def }}{=} \frac{1}{2}\left(\mathcal{L}^{\top}+\mathcal{L}\right)$ is a Laplacian of an undirected graph corresponding to $\mathcal{L}$, and $\mathbf{U}^{+}$is its Moore-Penrose pseudoinverse. This shows that it suffices to instead solve, $\mathcal{L}^{\top} \mathbf{U}^{+} \mathcal{L} x=\mathcal{L}^{\top} \mathbf{U}^{+} x$. Now using a nearly-linear-time Laplacian system solver, we can apply $\mathbf{U}^{+}$to a vector efficiently. As such, we simply need to show that we can efficiently solve systems in the symmetric matrix $\mathcal{L}^{\top} \mathbf{U}^{+} \mathcal{L}$

Next, we show that the matrix $\mathcal{L}^{\top} \mathbf{U}^{+} \mathcal{L}$ is, in an appropriate sense, approximable by $\mathbf{U}$. Formally we show that $\mathbf{U}$ is smaller in the sense that $\mathbf{U} \preceq \mathcal{L}^{\top} \mathbf{U}^{+} \mathcal{L}$, and that it is not too much larger in the sense that $\operatorname{tr}\left(\mathbf{U}^{+/ 2} \mathcal{L}^{\top} \mathbf{U}^{+} \mathcal{L} \mathbf{U}^{+/ 2}\right)=O\left(n^{2}\right)$. While the first proof is holds for a broad class of asymmetric matrices, to prove the second fact we exploit structure of Eulerian Laplacians, particularly the fact that an Eulerian graph has a decomposition into simple cycles.

Unfortunately, this property doesn't immediately yield an algorithm for solving Laplacian systems. The natural approach would be to use preconditioned Krylov methods, such as the Chebyshev method or conjugate gradient. These essentially apply a polynomial of $\mathbf{U}^{+} \mathcal{L}^{\top} \mathbf{U}^{+} \mathcal{L}$ to the right hand side. Unfortunately, Chebyshev iterations only yield a $\Omega(m n)$ time algorithm with this approach. For conjugate gradient, it can be shown that the trace bound leads to $o(m n)$ time algorithm in exact arithmetic, but, unfortunately, this analysis does not appear to be numerically stable, and we do not know how to show it yields this running time in our computational model.

Instead we implement an approach based on preconditioning and subsampling. We precondition

with $\mathcal{L}^{\top} \mathbf{U}^{+} \mathcal{L}+\alpha \mathbf{U}$ for a value of $\alpha$ we tune. This reduces the problem to only solving $\tilde{O}(\sqrt{\alpha})$ linear systems in $\mathcal{L}^{\top} \mathbf{U}^{+} \mathcal{L}+\alpha \mathbf{U}$. To solve these systems we note that we can write this equivalently as $\mathcal{L}^{\top} \mathbf{U}^{+} \mathbf{U} \mathbf{U}^{+} \mathcal{L}+\alpha \mathbf{U}$ and using the factorization of $\mathbf{U}$ into its edges we can subsample the inner $\mathbf{U}$ 
while preserving the matrix. Ultimately, this means we only need to solve systems in $\alpha \mathbf{U}$ plus a low rank matrix which we can do efficiently using the fact that there is an explicitly formula for low rank updates (i.e. Sherman-Morrison-Woodbury Matrix Identity). Trading between the number of such systems to solve, the preprocessing to solve these systems, and the time to solve them gives us our desired running time for solving such linear systems. We show in the appendix that we can, with some care, stably implement a preconditioned Chebyshev method and low rank update formulas. This allows us to circumvent the issues in using conjugate gradient and achieve our running time in the desired computational model.

\subsubsection{Solving RCDD Z-matrices}

A row column diagonal dominant (RCDD) matrix is simply a matrix $\mathbf{M}$ where $\mathbf{M}_{i i} \geq \sum_{j \neq i}\left|\mathbf{M}_{i j}\right|$

and $\mathbf{M}_{i i} \geq \sum_{j \neq i}\left|\mathbf{M}_{j i}\right|$ and a Z-matrix is a matrix $\mathbf{M}$ where the off-diagonal entries are negative. We show how to solve such matrices by directly reducing them to solving Eulerian Laplacian systems. Given a RCDD Z-matrix M, we add an additional row and column, filling in the entries in the natural way so that the resulting matrix is an Eulerian Laplacian. We show that, from the solution to such a linear system, we can immediately glean the solution to systems in $\mathbf{M}$. This reduction is analogous to the reduction from solving symmetric diagonally dominant (SDD) systems to solving undirected Laplacian systems. In the appendix we show that this method is stable.

\subsubsection{Computing the Stationary}

Given a RCDD Z-matrix solver we use it to compute the scaling that makes a directed Laplacian $\mathcal{L}$ Eulerian, i.e., we compute the stationary distribution. To do this, we pick an initial non-negative diagonal scaling $\mathbf{X}$ and a initial non-negative diagonal matrix $\mathbf{E}$ such that $(\mathbf{E}+\mathcal{L}) \mathbf{X}$ is $\alpha$-RCDD, that is each diagonal entry is a $1+\alpha$ larger in absolute value than the sum of the off-diagonal entries in both the corresponding row and column.

We then iteratively decrease $\mathbf{E}$ and update $\mathbf{X}$ while maintaining that the matrix is $\alpha$-RCDD. The key to this reduction is the fact that there is a natural way to perform a rank 1 update of $(\mathbf{E}+\mathcal{L}) \mathbf{X}$ to obtain an Eulerian Laplacian, and that the stationary distribution of this Laplacian can be obtained by solving a single system in $(\mathbf{E}+\mathcal{L}) \mathbf{X}$. Ultimately, this method yields a sequence of stationary distributions that, when multiplied together entrywise, yield a good approximate stationary distribution for $\mathcal{L}$. For a more detailed over this approach and this intuition underlying it, see Section 3.1.

\subsubsection{Additional Applications}

Our algorithms for computing personalized page rank vectors, solving linear systems in arbitrary RCDD matrices, and solving directed Laplacian linear systems are all proven in a similar fashion. We obtain an approximate stationary distribution, rescale the system to make it strictly RCDD, then solve it - all using algorithms from the previous sections in a black box fashion. Therefore, the running times for these applications - and in fact all our applications - depend solely (up to polylogarithmic factors) on the black-box costs of computing the stationary distribution and solving RCDD matrices.

However, our algorithms must determine how much accuracy to request when they invoke these two black-box routines. For computing personalized PageRank, one can determine the accuracy to request based solely on the restart probability. However, for our other applications, the accuracy our algorithms request has a dependence on the condition number $\kappa(\mathcal{L})$ of $\mathcal{L}$ and the ratio $\kappa\left(\mathbf{S}^{*}\right)$ of max over min stationary probability. In order to get an unconditional running time - and out of 
intrinsic interest - we show how to efficiently compute reasonable upper bounds on these quantities. We use an approach motivated by the close relationship of $\kappa(\mathcal{L})$ and mixing time. Specifically, we formulate a notion of personalized PageRank mixing time, then get a polynomially good estimate of this quantity using our ability to solve personalized PageRank. Finally, we show that $\kappa(\mathcal{L})$ and personalized pagerank mixing time are equivalent up to factors that are good enough ${ }^{4}$ for our purposes. With a reasonable bound on $\kappa(\mathcal{L})$, we are then able to choose a restart probability that is small enough in order to guarantee that personalized solving PageRank gives a good approximation of the stationary distribution.

Our algorithms for computing hitting times, escape probabilities, and all pairs commute times all start by taking a natural definition for the quantity of interest and massaging it into an explicit formula that has an $\mathcal{L}^{+}$in it. Then, they use various methods to approximately evaluate the formula. In the case of hitting times, we simply plug everything into the formula and invoke our approximate solver for $\mathcal{L}^{+}$with appropriately small error. Escape probabilities are handled similarly, except that there are also two unknown parameters which we show we can estimate to reasonable accuracy and plug in.

Perhaps the most sophisticated application is computing all pairs commute times. We show that the commute time from $u$ to $v$ is given by the simple formula $\left(\overrightarrow{1}_{u}-\overrightarrow{1}_{v}\right)^{\top}\left(\mathcal{L}_{b}^{\top} \mathbf{U}_{b}^{+} \mathcal{L}_{b}\right)^{+}\left(\overrightarrow{1}_{u}-\overrightarrow{1}_{v}\right)$ where $\mathcal{L}_{b}$ is the matrix obtained by performing the diagonal rescaling of $\mathcal{L}$ that turns its diagonal into the stationary distribution, which also makes the graph Eulerian. An interesting feature of this formula is that it involves applying the pseudo-inverse of a matrix of the very same form as the preconditioned system $\mathcal{L}^{\top} \mathbf{U}^{+} \mathcal{L}$ that our Eulerian Laplacian solver uses. Another interesting feature is that when $\mathcal{L}$ is symmetric, this formula simplifies to $\left(\overrightarrow{1}_{u}-\overrightarrow{1}_{v}\right)^{\top} \mathbf{U}_{b}^{+}\left(\overrightarrow{1}_{u}-\overrightarrow{1}_{v}\right)=2 m \cdot\left(\overrightarrow{1}_{u}-\overrightarrow{1}_{v}\right)^{\top} \mathbf{U}^{+}\left(\overrightarrow{1}_{u}-\overrightarrow{1}_{v}\right)$. Thus, it is a generalization of the well-known characterization of commute times in terms of effective resistance from undirected graphs. In undirected graphs, all pairs commute times can be computed efficiently via Johnson-Lindenstrauss sketching [54]. We show that a similar approach extends to directed Laplacians as well. While the general approach is similar, the error analysis is complicated by the fact that we only have access to an approximate stationary distribution. If this were used naively, one would have to deal with an approximate version of $\mathcal{L}_{b}$ that, importantly, is only approximately Eulerian. We bypass this issue by showing how to construct an Eulerian graph whose stationary is exactly known and whose commute times approximate the commute times of the original graph. This may be of independent interest.

The fact that a matrix of the form $\mathcal{L}^{\top} \mathbf{U}^{+} \mathcal{L}$ comes up both in solving Eulerian Laplacians and in sketching commute times indicates that it is an even more natural object than it might appear at first.

\subsection{Paper Organization}

The rest of our paper is organized as follows:

- Section 2 - we cover preliminary information

- Section 3 - we show how to compute the stationary distribution

- Section 4 - we provide our fast Eulerian Laplacian system solver

- Section 5 - we reduce strict RCDD linear systems to solving Eulerian systems

\footnotetext{
${ }^{4}$ They are equivalent up to factors polynomial in $n$ and themselves. Since these quantities appear only in logs in our runtimes and these logs already have factors of $n$ in them, this polynomial equivalence only changes runtimes by a constant factor compared to if we had exact estimates. A similar relationship also holds between $\kappa(\mathcal{L})$ and the mixing time of lazy random walks on the graph. (See Section 6 for details.)
} 
- Section 6 - we provide condition number quantities for applications and prove equivalences

- Section 7 - we provide our applications

- Appendix A - we discuss numerical stability of our solvers

- Appendix B - we provide facts about matrices we use throughout

- Appendix C - we derive identities for hitting times, commute times, and escape probabilities

\section{Preliminaries}

In this section we introduce notation and provide basic machinery we use throughout this paper.

\subsection{Notation}

Matrices: We use bold to denote matrices and let $\mathbf{I}_{n}, \mathbf{0}_{n} \in \mathcal{R}^{n \times n}$ denote the identity matrix and zero matrix respectively. For symmetric matrices $\mathbf{A}, \mathbf{B} \in \mathcal{R}^{n \times n}$ we use $\mathbf{A} \preceq \mathbf{B}$ to denote the condition that $x^{\top} \mathbf{A} x \leq x^{\top} \mathbf{B} x$ and we define $\succeq, \prec$, and $\succ$ analogously. We call a symmetric matrix $\mathbf{A} \in \mathcal{R}^{n \times n}$ positive semidefinite if $\mathbf{A} \succeq \mathbf{0}_{n}$ and we let $\|x\|_{\mathbf{A}} \stackrel{\text { def }}{=} \sqrt{x^{\top} \mathbf{A} x}$. For any norm $\|\cdot\|$ define on vectors in $\mathcal{R}^{n}$ we define the operator norm it induces on $\mathcal{R}^{n \times n}$ by $\|\mathbf{A}\|=\max _{x \neq 0} \frac{\|\mathbf{A} x\|}{\|x\|}$ for all $\mathbf{A} \in \mathcal{R}^{n \times n}$.

Diagonals: For $x \in \mathcal{R}^{n}$ we let $\operatorname{diag}(x) \in \mathcal{R}^{n \times n}$ denote the diagonal matrix with $\operatorname{diag}(x)_{i i}=x_{i}$ and when it is clear from context we more simply write $\mathbf{X} \stackrel{\text { def }}{=} \operatorname{diag}(x)$. For $\mathbf{A} \in \mathcal{R}^{n \times n}$ we $\operatorname{let} \operatorname{diag}(\mathbf{A})$ denote the vector corresponding to the diagonal of $\mathbf{A}$ and we let $\operatorname{diag}(\mathbf{A}) \stackrel{\operatorname{def}}{=} \operatorname{diag}(\operatorname{diag}(\mathbf{A}))$, i.e. $\mathbf{A}$ with the off-diagonal set to 0 .

Vectors: We let $\overrightarrow{0}_{n}, \overrightarrow{1}_{n} \in \mathcal{R}^{n}$ denote the all zeros and ones vectors respectively. We use $\overrightarrow{1}_{i} \in \mathcal{R}^{n}$ to denote the indicator vector for coordinate $i \in[n]$, i.e. $\left[\overrightarrow{1_{i}}\right]_{j}=0$ for $j \neq i$ and $\left[\overrightarrow{1}_{i}\right]_{i}=1$. Occasionally we apply scalar operations to vectors with the interpretation that they should be applied coordinate-wise, e.g. for $x, y \in \mathcal{R}^{n}$ we let $\max \{x, y\}$ denote the vector $z \in \mathcal{R}^{n}$ with $z_{i}=\max \left\{x_{i}, y_{i}\right\}$ and we use $x \geq y$ to denote the condition that $x_{i} \geq y_{i}$ for all $i \in[n]$.

Condition Numbers: Given a invertible matrix $\mathbf{A} \in \mathcal{R}^{n \times n}$ we let $\kappa(\mathbf{A}) \stackrel{\text { def }}{=}\|\mathbf{A}\|_{2} \cdot\left\|\mathbf{A}^{+}\right\|_{2}$ denote the condition number of $\mathbf{A}$. Note that if $\mathbf{X} \in \mathcal{R}^{n \times n}$ is a nonzero diagonal matrix then $\kappa(\mathbf{X})=\frac{\max _{i \in[n]}\left|\mathbf{X}_{i i}\right|}{\min _{i \in[n]}\left|\mathbf{X}_{i i}\right|}$.

Sets: We let $[n] \stackrel{\text { def }}{=}\{1, \ldots, n\}$ and $\Delta^{n} \stackrel{\text { def }}{=}\left\{x \in \mathcal{R}_{\geq 0}^{n} \mid \overrightarrow{1}_{n}^{\top} x=1\right\}$, i.e. the $n$-dimensional simplex.

\subsection{Matrix Classes}

Diagonal Dominance: We call a possibly asymmetric matrix $\mathbf{A} \in \mathcal{R}^{n \times n} \alpha$-row diagonally dominant $(R D D)$ if $\mathbf{A}_{i i} \geq(1+\alpha) \sum_{j \neq i}\left|\mathbf{A}_{i j}\right|$ for all $i \in[n]$, $\alpha$-column diagonally dominant (CDD) if $\mathbf{A}_{i i} \geq(1+\alpha) \sum_{j \neq i}\left|\mathbf{A}_{j i}\right|$, and $\alpha-R C D D$ if it is both $\alpha$-RDD and $\alpha$-CDD. For brevity, we call $\mathbf{A}$ $\mathrm{RCDD}$ if it is 0 -RCDD and strictly RCDD if it is $\alpha$-RCDD for $\alpha>0$.

Z-matrix: A matrix $\mathbf{M} \in \mathcal{R}^{n \times n}$ is called a Z-matrix if $\mathbf{M}_{i j} \leq 0$ for all $i \neq j$, i.e. every offdiagonal entry is non-positive. 
Directed Laplacian: A matrix $\mathcal{L} \in \mathcal{R}^{n \times n}$ is called a directed Laplacian if it a Z-matrix with $\overrightarrow{1}_{n}^{\top} \mathcal{L}=\overrightarrow{0}_{n}$, that is $\mathcal{L}_{i j} \leq 0$ for all $i \neq j$ and $\mathcal{L}_{i i}=-\sum_{j \neq i} \mathcal{L}_{j i}$ for all $i$. To every directed Laplacian $\mathcal{L}$ we associate a graph $G_{\mathcal{L}}=(V, E, w)$ with vertices $V=[n]$ and an edge $(i, j)$ of weight $w_{i j}=-\mathcal{L}_{j i}$ for all $i \neq j \in[n]$ with $\mathcal{L}_{j i} \neq 0$. Occasionally we write $\mathcal{L}=\mathbf{D}-\mathbf{A}^{\top}$ to denote that we decompose $\mathcal{L}$ into the diagonal matrix $\mathbf{D}$ where $\mathbf{D}_{i i}=\mathcal{L}_{i i}$ is the out degree of vertex $i$ in $G_{\mathcal{L}}$ and $\mathbf{A}$ is weighted adjacency matrix of $G_{\mathcal{L}}$ with $\mathbf{A}_{i j}=w_{i j}$ if $(i, j) \in E$ and $\mathbf{A}_{i j}=0$ otherwise. We call $\mathbf{W}=\mathbf{A}^{\top} \mathbf{D}^{-1}$ the random walk matrix associated with $G_{\mathcal{L}}$. We call $\mathcal{L}$ Eulerian if additionally $\mathcal{L} \overrightarrow{1}_{n}=\overrightarrow{0}_{n}$ as in this case the associated graph $G_{\mathcal{L}}$ is Eulerian.

(Symmetric) Laplacian: A matrix $\mathbf{U} \in \mathcal{R}^{n \times n}$ is called a Symmetric Laplacian or just a Laplacian if it is symmetric and a Laplacian. This coincides with the standard definition of Laplacian and in this case note that the associated graph $G_{\mathbf{U}}=(V, E, w)$ is symmetric. For a Laplacian we also associate a matrix $\mathbf{B} \in \mathcal{R}^{E \times V}$ known as the weighted incidence matrix. Each row $b^{(i)}$ of $\mathbf{B}$ corresponds to an edge $\{j, k\} \in E$ and for a canonical orientation ordering of $\{j, k\}$ we have $b_{j}^{(i)}=\sqrt{w_{\{j, k\}}}$, $b_{k}^{(i)}=-\sqrt{w_{\{j, k\}}}$, and $b_{l}^{(i)}=0$ if $l \notin\{j, k\}$. Note that $\mathbf{U}=\mathbf{B}^{\top} \mathbf{B}$ and thus $\mathcal{L}$ is always PSD.

Random Walk Matrix: A matrix $\mathbf{W} \in \mathcal{R}^{n \times n}$ is called a random walk matrix if $\mathbf{W}_{i j} \geq 0$ for all $i, j \in[n]$ and $\overrightarrow{1}_{n}^{\top} \mathbf{W}=\overrightarrow{1}_{n}$. To every random walk matrix $\mathbf{W}$ we associated a directed graph $G_{\mathbf{W}}=(V, E, w)$ with vertices $V=[n]$ and an edge from $i$ to $j$ of weight $w_{i j}=\mathbf{W}_{i j}$ for all $i, j \in[n]$ with $\mathbf{W}_{i j} \neq 0$. Note if we say that $\mathcal{L}=\mathbf{I}-\mathbf{W}$ is a directed Laplacian, then $\mathbf{W}$ is a random walk matrix and the directed graphs associated with $\mathcal{L}$ and $\mathbf{W}$ are identical.

Lazy Random Walk Matrix: Given a random walk matrix $\mathbf{W} \in \mathcal{R}^{n \times n}$ the $\alpha$-lazy random walk matrix associated with $\mathbf{W}$ for $\alpha \in[0,1]$ is given by $\alpha \mathbf{I}+(1-\alpha) \mathbf{W}$. When $\alpha=\frac{1}{2}$ we call this a lazy random walk matrix for short and typically denote it $\widetilde{\mathbf{W}}$.

Personalized PageRank Matrix: Given a random walk matrix $\mathbf{W} \in \mathcal{R}^{n \times n}$ the personalized PageRank matrix with restart probability $\beta \in[0,1]$ is given by $\mathbf{M}_{p p(\beta)}=\beta(\mathbf{I}-(1-\beta) \mathbf{W})^{-1}$. Given any probability vector $p \in \Delta^{n}$ the personalized PageRank vector with restart probability $\beta$ and vector $p$ is the vector $x$ which satisfies $\beta p+(1-\beta) \mathbf{W} x=x$. Rearranging terms we see that $x=\mathbf{M}_{\beta} p$ hence justifying our naming of $\mathbf{M}_{p p(\beta)}$ as the personalized PageRank matrix.

\subsection{Directed Laplacians of Strongly Connected Graphs}

Here we give properties of a directed Laplacian, $\mathcal{L} \in \mathcal{R}^{n \times n}$, whose associated graph, $G_{\mathcal{L}}$, is strongly connected that we use throughout the paper. Formally, we provide Lemma 1 which shows that $\mathcal{L}$ always has a positive Eulerian scaling, that is a $x \in \mathcal{R}_{>0}$ with $\mathcal{L} \mathbf{X}$ Eulerian, and that this is given by the stationary distribution of the associated random walk matrix. Using this we completely characterize the kernel of $\mathcal{L}$ and $\mathcal{L}^{\top}$.

Lemma 1. For directed Laplacian $\mathcal{L}=\mathbf{D}-\mathbf{A}^{\top} \in \mathcal{R}^{n \times n}$ whose associated graph is strongly connected there exists a positive vector $s \in \mathcal{R}_{>0}^{n}$ (unique up to scaling) such that the following equivalent conditions hold.

- $\mathbf{W} s=s$ for the random walk matrix $\mathbf{W}=\mathbf{A}^{\top} \mathbf{D}^{-1}$ associated with $\mathcal{L}$.

- $\mathcal{L} \mathbf{D}^{-1} s=0$ 
- $\mathcal{L} \mathbf{D}^{-1} \mathbf{S}$ for $\mathbf{S}=\operatorname{diag}(s)$ is an Eulerian Laplacian.

If we scale $s$ so that $\|s\|_{1}=1$ then we call $s$ the stationary distribution associated with the random walk on the associated graph $G_{\mathcal{L}}$. We call any vector $x \in \mathcal{R}_{>0}^{n}$ such that $\mathcal{L} \mathbf{X}$ is an Eulerian Laplacian an eulerian scaling for $\mathcal{L}$. Furthermore, $\operatorname{ker}(\mathcal{L})=\operatorname{span}\left(\mathbf{D}^{-1} s\right)$ and $\operatorname{ker}\left(\mathcal{L}^{\top}\right)=\operatorname{span}\left(\overrightarrow{1}_{n}\right)$.

Proof. Since the graph associated with $\mathcal{L}$ is strongly connected we have that for any $\alpha>0$ note that $\alpha \mathbf{I}+\mathbf{W}$ is positive irreducible and aperiodic with all columns having $\ell_{1}$ norm of $1+\alpha$. Therefore, by the Perron-Frobenius Theorem we know and therefore therefore there exists a positive vector $s \in \mathcal{R}_{>0}^{n}$ unique up to scaling such that that $(\alpha \mathbf{I}+\mathbf{W}) s=(\alpha+1) s$ or equivalently $\mathbf{W} s=s$. Therefore, by definition $\mathbf{A}^{\top} \mathbf{D}^{-1} s=s$ and $\mathcal{L} \mathbf{D}^{-1} s=\left(\mathbf{D}-\mathbf{A}^{\top}\right) \mathbf{D}^{-1} s=0$. Furthermore this implies that for $\mathcal{L}^{\prime}=\mathcal{L} \mathbf{D}^{-1} \mathbf{S}$ we have $\mathcal{L}^{\prime} \overrightarrow{1}_{n}=\overrightarrow{0}_{n}$ and as $\mathcal{L}$ is Z-matrix so is $\mathcal{L}^{\prime}$ and therefore $\mathcal{L}^{\prime}$ is a Z-matrix. Furthermore clearly $\mathcal{L}^{\top} \mathbf{D}^{-1} \mathbf{S}$ being a Eulerian Laplacian implies $\mathbf{W} s=s$ and therefore we see that the conditions are all equivalent. Lastly, we know that $\frac{1}{2}\left(\mathcal{L}^{\prime}+\left[\mathcal{L}^{\prime}\right]^{\top}\right)$ is a symmetric Laplacian associated with an connected graph. Therefore it is PSD having only $\overrightarrow{1}_{n}$ in there kernel yielding our characterization of the kernel of $\mathcal{L}$ and $\mathcal{L}^{\top}$.

Note that Lemma 1 immediately implies that any random walk matrix $\mathbf{W}$ associated with a strongly connected graph has a unique stationary distribution $s$, i.e. $s \in \Delta^{n}$ and $\mathbf{W} s=s$. Furthermore, we see that all $\alpha$-lazy random walks for $\alpha \in[0,1)$ associated with $\mathbf{W}$ have the same stationary distribution.

\section{Computing the Stationary Distribution}

Here we show to compute the stationary distribution given an $\alpha$-RCDD Z-matrix linear system solver. Throughout this section, we let $\mathcal{L}=\mathbf{D}-\mathbf{A}^{\top} \in \mathcal{R}^{n \times n}$ denote a directed Laplacian and our primary goal in this section is to compute an approximate stationary vector $s \in \mathcal{R}_{>0}^{V}$ such that $\mathcal{L} \mathbf{D}^{-1} \mathbf{S}$ is approximately Eulerian. The main result of this section is the following:

Theorem 2 (Stationary Computation Using a RCDD Solver). Given $\alpha \in\left(0, \frac{1}{2}\right)$ and $\mathcal{L} \in \mathcal{R}^{n \times n}, a$ directed Laplacian with $m$ nonzero-entries, in time $O\left((m+\mathcal{T}) \cdot \log \alpha^{-1}\right)$ Algorithm 1 computes an approximate stationary distribution $s \in \Delta^{n}$ such that $(3 \alpha n \cdot \mathbf{D}+\mathcal{L}) \mathbf{D}^{-1} \mathbf{S}$ is $\alpha-R C D D$ where $\mathbf{D}=$ $\operatorname{diag}(\mathcal{L}), \mathbf{S}=\operatorname{diag}(s)$, and $\mathcal{T}$ is the cost of computing an $\epsilon$-approximate solution to a $n \times n \alpha-R C D D$ $Z$-matrix linear system with m-nonzero entries, i.e. computing $x$ such that $\left\|x-\mathbf{M}^{-1} b\right\|_{\operatorname{diag}(\mathbf{M})} \leq$ $\frac{\epsilon}{\alpha}\|b\|_{\operatorname{diag}(\mathbf{M})^{-1}}$ for $\alpha-R C D D$ Z-matrix $\mathbf{M} \in \mathcal{R}^{n \times n}$ with $O(m)$ non-zero entries, $\epsilon=O(\operatorname{poly}(n / \alpha))$. Furthermore, $\kappa(\mathbf{S}) \leq \frac{20}{\alpha^{2}} n$, where $\kappa(\mathbf{S})$ is the ratio between the largest and smallest elements of $s$, and the diagonals of all intermediate $R C D D$ systems also have condition number $O(\operatorname{poly}(n / \alpha))$.

The remainder of this section is divided as follows:

- Section 3.1: we provide an overview of our approach to computing the stationary distribution

- Section 3.2: we provide the details of our algorithm

- Section 3.3: we analyze this algorithm proving Theorem 2.

\subsection{The Approach}

Our approach to computing the stationary distribution is broadly related to the work of Daitch and Spielman [12] for computing the diagonal scaling makes a symmetric $M$-matrix diagonally 
dominant. However, the final rescaling that we're trying to calculate is given by the uniqueness of the stationary distribution, which in turn follows from the Perron-Frobenius theorem. The fact that we're rescaling and computing on the same objective leads a more direct iterative process.

As in [12] we use an iterative that brings a matrix increasingly close to being RCDD. However, Our convergence process is through potential functions instead of through combinatorial entry count; instead of making parts of $\mathcal{L}$ RCDD incrementally we instead start with a relaxation of $\mathcal{L}$ that is RCDD and iteratively bring this matrix closer to $\mathcal{L}$ while maintaining that it is RCDD. We remark that this scheme can also be adapted to find rescalings of symmetric M-matrices.

Our algorithm hinges on two key insights. The first is that if we have positive vectors $e, x \in \mathcal{R}_{>0}^{n}$ so that $\mathbf{M} \stackrel{\text { def }}{=}(\mathbf{E}+\mathcal{L}) \mathbf{X}$ is $\alpha$-RCDD, then for any vector $g \in \mathcal{R}_{>0}^{n}$ with $\|g\|_{1}=1$ we can compute the stationary distribution of the directed Laplacian $\mathcal{L}^{\prime}=\mathbf{E}-g e^{\top}+\mathcal{L}$ by solving a single linear system in $\mathbf{M}$. Note that $\mathbf{M}$ is clearly invertible (Lemma 4) whereas $\mathcal{L}^{\prime}$ has a kernel (Lemma 1). Furthermore, $\mathcal{L}^{\prime} \mathbf{X}$ is simply a rank-1 update of $\mathbf{M}$ and consequently there kernel $\mathcal{L}^{\prime}$ has a closed form, namely $\mathbf{X} \mathcal{L}^{-1} f$ (Lemma 3). Since the stationary distribution of $\mathcal{L}^{\prime}$ is given by its kernel (Lemma 1) we obtain the result.

This first insight implies that if we have a RCDD Z-matrix we can compute the stationary of a related matrix, however a priori it is unclear how this allows to compute the stationary of $\mathcal{L}$. The second insight is that if we compute the stationary of $\mathcal{L}^{\prime}$, e.g. we compute some $y \in \mathcal{R}_{>0}^{n}$ such that $\mathcal{L}^{\prime} \mathbf{Y}$ is an Eulerian Laplacian, then $(\mathbf{E}+\mathcal{L}) \mathbf{Y}$ is strictly $\mathrm{RCDD}$. Since $\mathcal{L}^{\prime} \mathbf{Y}$ is Eulerian, $\left(\mathbf{E}-\mathcal{L}-\mathcal{L}^{\prime}\right) \mathbf{Y}=g e^{\top} \mathbf{Y}$ is an all positive matrix which is entrywise less than $\mathcal{L}^{\prime}$ in absolute value. In other words, removing $g e^{\top} \mathbf{Y}$ from $\mathcal{L}^{\prime} \mathbf{Y}$ strictly decreases the absolute value of the off diagonal entries and increases the value of the diagonal entries causing $(\mathbf{E}+\mathcal{L}) \mathbf{Y}$ to be strictly RCDD. Consequently, given $y$ we can hope to decrease $e$ to achieve $e^{\prime}$ to obtain an $\alpha$-RCDD Z-matrix $\mathbf{M}^{\prime}=\left(\mathbf{E}^{\prime}+\mathcal{L}\right) \mathbf{Y}$ where $e^{\prime} \leq e$.

Combining these insights naturally yields our algorithm. We compute an initial $\alpha$-RCDD Zmatrix $\mathbf{M}=(\mathbf{E}+\mathcal{L}) \mathbf{X}$ and compute $x^{\prime}=\mathbf{X M}^{-1} g$ for $g \in \mathcal{R}_{>0}^{n}$ with $\|g\|_{1}=1$ so that $\mathcal{L}^{\prime}=$ $\left(\mathbf{E}-g e^{\top}+\mathcal{L}\right) \mathbf{X}^{\prime}$ is Eulerian. We then compute the smallest $e^{\prime} \in \mathcal{R}_{>0}^{n}$ so that $\left(\mathbf{E}^{\prime}+\mathcal{L}\right) \mathbf{X}^{\prime}$ is again $\alpha$-RCDD and repeat. What remains to show is that for proper choice of initial $x$ and $e$ as well as proper choice of $g$ in each iteration, this algorithm converges quickly provided we use a sufficiently accurate RCDD linear system solver.

The only missing ingredient for such analysis, is how to measure progress of the algorithm. Note that $(\mathbf{E}+\mathcal{L}) \mathbf{X}$ is $\alpha$-CDD if and only if $e \geq \alpha d$ (Lemma 6) and consequently the smallest $\alpha d$ is the smallest value of $e$ we can hope for to keep $(\mathbf{E}+\mathcal{L}) \mathbf{X} \alpha$-RCDD. We show the each iteration of the procedure outlined above brings $e$ rapidly closer to $\alpha \mathrm{d}$. In particular we show that in each iteration we can ensure that $\left\|\mathbf{D}^{-1}(e-\alpha d)\right\|_{1}$ decreases by a multiplicative constant up to a small additive factor depending only on $\alpha$ (Lemma 10). Putting this all together we achieve an algorithm (See Section 3.2) that in $O\left(\ln \left(\frac{1}{\alpha}\right)\right)$ iterations finds the desired $x, e$.

\subsection{The Algorithm}

Following the approach outlined in the previous section, our iterative algorithm for computing the stationary is given by Algorithm 1. First, we compute positive vectors $e^{(0)}, x^{(0)} \in \mathcal{R}_{>0}^{V}$ such that and $\mathbf{M}^{(0)} \stackrel{\text { def }}{=}\left(\mathbf{E}^{(0)}+\mathcal{L}\right) \mathbf{X}^{(0)}$ is $\alpha$-DD. In particular, we let $x^{(0)} \stackrel{\text { def }}{=} \mathbf{D}^{-1} 1$ and we let $e^{(0)} \in \mathcal{R}_{\geq 0}^{V}$ be the entry-wise minimal non-negative vector such that $\mathbf{M}^{(0)}$ is $\alpha$-RCDD. This choice of $x^{(0)}$ allows us to reason about the size of $e^{(0)}$ easily (See Lemma 7).

Next, we iterate, computing $e^{(t)}, x^{(t)} \in \mathcal{R}_{\geq 0}^{V}$ such that $\mathbf{M}^{(t)}=\left(\mathbf{E}^{(t)}+\mathcal{L}\right) \mathbf{X}^{(t)}$ is $\alpha$-RCDD. We pick the $g^{(t)}$ discussed in the previous section to be $g^{(t)}=\left\|\mathbf{D}^{-1} e^{(t)}\right\|_{1}^{-1} \mathbf{D}^{-1} e^{(t)}$ as this naturally corre- 


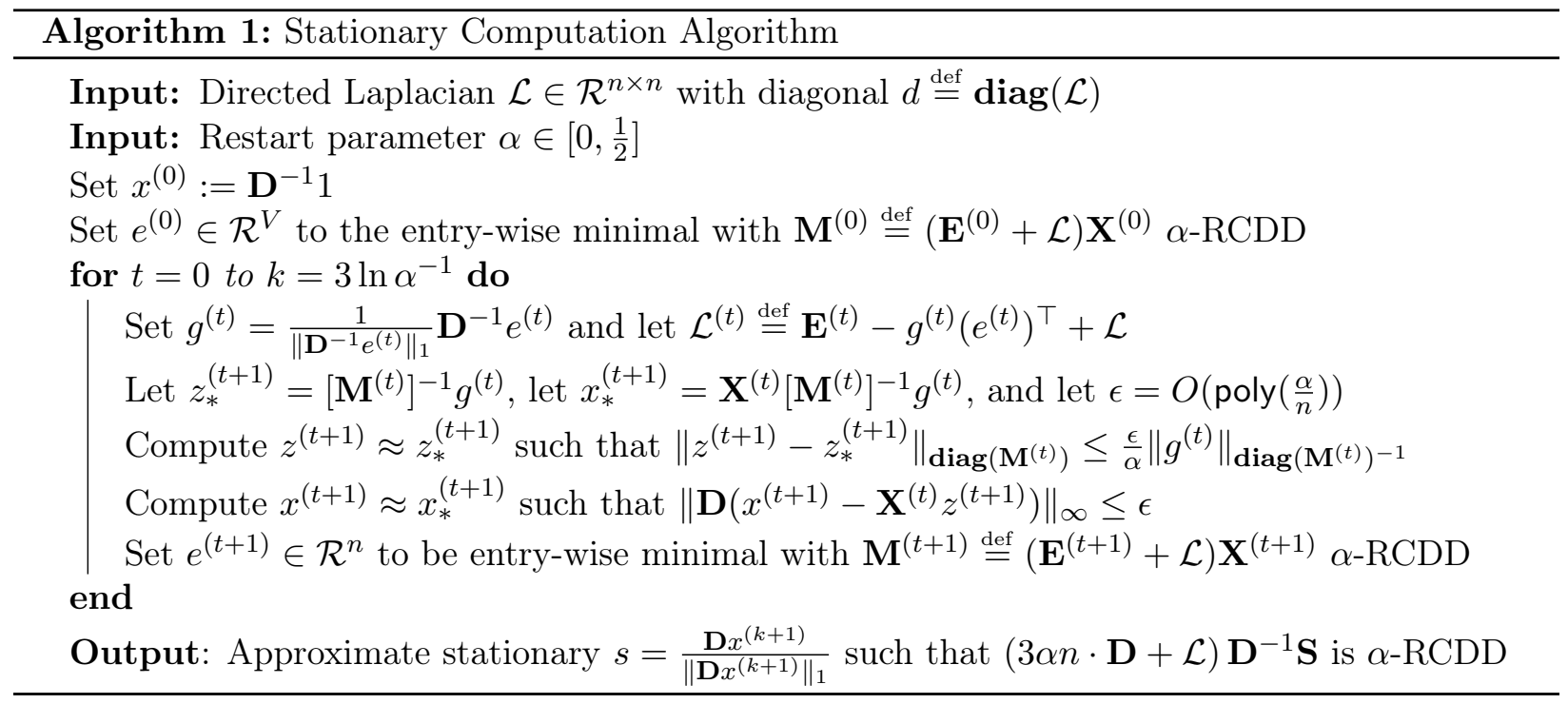

sponds to the relative amount of each $e^{(t)}$ we want to remove. We then let $x^{(t+1)} \approx \mathbf{X}^{(t)}\left[\mathbf{M}^{(t)}\right]^{-1} g^{(t)}$ so that $\mathcal{L}^{(t)} \stackrel{\text { def }}{=} \mathbf{E}^{(t)}-g^{(t)}\left[e^{(t)}\right]^{\top}+\mathcal{L}$ is a directed Laplacian where $\mathcal{L}^{(t+1)} \mathbf{X}^{(t+1)}$ is nearly Eulerian. We let $e^{(t+1)} \in \mathcal{R}_{\geq 0}^{V}$ be the entry-wise minimal vector such that $\mathbf{M}^{(t+1)}$ is $\alpha$-RCDD and then we repeat. Outputting the final $x^{(t)}$ computed completes the result.

To complete the specification of the algorithm all that remains is discuss the precision with which we need to carry out the operations of the algorithm. There are two places in particular where we might worry that error in inexact arithmetic could hinder the progress of the algorithm. The first is the degree of precision to which we solve the linear system in $\mathbf{M}^{(t)}$, i.e. compute $z_{*}^{(t+1)} \stackrel{\text { def }}{=}\left[\mathbf{M}^{(t)}\right]^{-1} g^{(t)}$. We show that computing instead an approximate $z^{(t+1)}$ such that $\| z^{(t+1)}-$ $z_{*}\left\|_{\operatorname{diag}\left(\mathbf{M}^{(t)}\right)} \leq \frac{\epsilon}{\alpha}\right\| g^{(t+1)} \|_{\operatorname{diag}\left(\mathbf{M}^{(t)}\right)^{-1}}$ for $\epsilon=O\left(\operatorname{poly}\left(\frac{\alpha}{n}\right)\right)$ suffices. The second is in computing $x^{(t+1)}=\mathbf{X}^{(t)} z^{(t+1)}$. Unrolling the iterations of the algorithm we see that $x^{(t+1)}$ is the entry-wise product of all previous vectors $\left[\mathbf{M}^{\left(t^{\prime}\right)}\right]^{-1} g^{\left(t^{\prime}\right)}$ with $t^{\prime} \leq t$. Consequently, one might worry that since that errors could accumulate. We show that computing a bounded precision $x^{(t+1)}$ such that $\left\|\mathbf{D}\left(x^{(t+1)}-\mathbf{X}^{(t)} z^{(t+1)}\right)\right\|_{\infty} \leq \epsilon$ for $\epsilon=O\left(\right.$ poly $\left.\left(\frac{\alpha}{n}\right)\right)$ suffices.

\subsection{The Analysis}

Here we prove Theorem 2 by showing the correctness of our algorithm for computing the stationary distribution, Algorithm 1. We split our proof into multiple parts:

- Section 3.3.1: we show that $x_{*}^{(t+1)}$ is indeed a positive vector that makes $\mathcal{L}^{(t)} \mathbf{X}_{*}^{(t+1)}$ Eulerian

- Section 3.3.2: we provide bounds on $e^{(t)}$

- Section 3.3.3: we show how much $\left\|\mathbf{D}^{-1}\left(e^{(t)}-\alpha d\right)\right\|_{1}$ decreases between iterations

- Section 3.3.4: we bound the error induced by solving linear systems approximately

- Section 3.3.3: we put everything together to prove Theorem 2.

Note that much of the analysis is done in slightly greater generality then required. In particular, much of our analysis works so long as $\alpha \geq 0$ however we constrain $\alpha \in\left(0, \frac{1}{2}\right)$ so we can easily reason 
about the stability of the procedure, measure of the quality of the output stationary distribution, and simplify our analysis. Also note that minimal effort was taken to control the actual value of $\epsilon$ beyond to simplify the proof that $\epsilon=O\left(\operatorname{poly}\left(\frac{\alpha}{n}\right)\right)$ suffices.

\subsubsection{Properties of $x$}

Here we prove that $x_{*}^{(t+1)}$ is indeed a positive vector such that $\mathcal{L}^{(t)} \mathbf{X}_{*}^{(t+1)}$ is Eulerian. First we provide two general lemmas about the kernel of a matrix after a rank one update, Lemma 3, and the invertibility of $\alpha$-DD matrices Lemma 4. Then, using these lemmas we prove the main result of this subsection, Lemma 5 , which yields our desired properties of $x_{*}^{(t+1)}$.

Lemma 3. $\operatorname{ker}\left(\mathbf{M}+u v^{\top}\right) \subseteq \operatorname{span}\left(\mathbf{M}^{-1} u\right)$ for all invertible $\mathbf{M} \in \mathcal{R}^{n \times n}$ and $u, v \in \mathcal{R}^{n}$.

Proof. If $\operatorname{ker}\left(\mathbf{M}+u v^{\top}\right)=\emptyset$ the claim follows trivially. Otherwise, there is $x \in \mathcal{R}^{n}$ with $x \neq 0$ such that $\left(\mathbf{M}+u v^{\top}\right) x=0$. Since $\mathbf{M}$ is invertible it follows that $x=-\mathbf{M}^{-1} u\left(v^{\top} x\right) \in \operatorname{span}\left(\mathbf{M}^{-1} u\right)$.

Lemma 4. Every strictly RCDD matrix is invertible.

Proof. Let $\mathbf{M}$ be an arbitrary strictly RCDD matrix. By definition, $\mathbf{M}$ is $\alpha$-RCDD for some $\alpha>0$. Consequently, there exists $\epsilon>0$ such that $\mathbf{N}=\mathbf{M}-\epsilon \mathbf{I}$ is $\beta$-RCDD for some $\beta>0$. Now,

$$
\mathbf{M}^{\top} \mathbf{M}=(\epsilon \mathbf{I}+\mathbf{N})^{\top}(\epsilon \mathbf{I}+\mathbf{N})=\epsilon^{2} \mathbf{I}+\epsilon\left(\mathbf{N}+\mathbf{N}^{\top}\right)+\mathbf{N}^{\top} \mathbf{N}
$$

However, $\mathbf{N}+\mathbf{N}^{\top}$ is clearly a $\beta$-RCDD symmetric matrix and therefore is PSD. Consequently $\mathbf{M}^{\top} \mathbf{M} \succeq \epsilon^{2} \mathbf{I}$ and therefore $\mathbf{M}$ doesn't have a non-trivial kernel and is invertible.

Lemma 5. Let $x, e \in \mathcal{R}_{>0}^{n}$ be such that $\mathbf{M}=(\mathbf{E}+\mathcal{L}) \mathbf{X}$ is strictly $R C D D$ and let $g \in \mathcal{R}_{>0}^{n}$ with $\|g\|_{1}=1$. Then $x_{*} \stackrel{\text { def }}{=} \mathbf{X M}^{-1} g$ is all positive and $\left(\mathbf{E}-g e^{\top}+\mathcal{L}\right) \mathbf{X}_{*}$ is an Eulerian Laplacian.

Proof. Since $\mathbf{M}$ is strictly RCDD by Lemma 4 it is invertible. Furthermore, since $x, e, g \geq 0$ by Lemma 62 we know that $\mathbf{M}^{-1} g>0$ and $x_{*}>0$. Now $\mathcal{L}^{\prime}=\left(\mathbf{E}-g e^{\top}+\mathcal{L}\right) \mathbf{X}$ is a directed Laplacian and therefore has a non-trivial right kernel. Consequently, by Lemma 3 and the fact that $\mathcal{L}^{\prime}-\mathbf{M}=$ $-g e^{\top} \mathbf{X}$ we know that $\mathbf{M}^{-1} g$ is in the kernel of $\mathcal{L}^{\prime}$. Consequently, $\left(\mathbf{E}-g e^{\top}+\mathcal{L}\right) \mathbf{X} \mathbf{M}^{-1} \mathbf{G} 1=0$ yielding the result.

\subsubsection{Properties of $e$}

Here we provide bounds on what $e^{(t)}$ needs to be for $\mathbf{M}^{(t)}$ to be $\alpha$-RCDD. First in Lemma 6 we give necessary and sufficient conditions for $(\mathbf{E}+\mathcal{L}) \mathbf{X}$ to be $\alpha$-CDD. This provides a lower bound on all the $e^{(t)}$. Then in Lemma 7 we upper bound $e^{(0)}$. We conclude with Lemma 8 , which yields a closed formula for the $e^{(t)}$.

Lemma 6 (Conditions for $\alpha$-CDD). For all vectors $x, e \in \mathcal{R}_{>0}^{n}$ and directed Laplacian $\mathcal{L} \in \mathcal{R}^{n \times n}$ the matrix $(\mathbf{E}+\mathcal{L}) \mathbf{X}$ is $\alpha-C D D$ if and only if $e \geq \alpha$ d.

Proof. By definition of $\mathcal{L}=\mathbf{D}-\mathbf{A}$ we have that $(\mathbf{E}+\mathcal{L}) \mathbf{X}$ is column $\alpha$-CDD if and only if entrywise

$$
1^{\top}(\mathbf{E}+\mathbf{D}) \mathbf{X} \geq(1+\alpha) 1^{\top} \mathbf{A}^{\top} \mathbf{X}=(1+\alpha) d^{\top} \mathbf{X} .
$$

Applying $\mathbf{X}^{-1}$ to each side and then subtracting $d^{\top}$ from each side yields the result.

Lemma 7 (Bound on $\left.e^{(0)}\right) \cdot\left\|\mathbf{D}^{-1} e^{(0)}\right\|_{1} \leq(1+2 \alpha) n$ 
Proof. Since $e^{(0)}$ is the entry-wise minimal vector such that $\mathbf{M}^{(0)}=\left(\mathbf{E}^{(0)}+\mathcal{L}\right) \mathbf{X}^{(0)}$ is $\alpha$-RCDD and since $\mathbf{X}^{(0)}=\mathbf{D}^{-1}$ we have

$$
\mathbf{D}^{-1}\left(e^{(0)}+d\right)=(1+\alpha) \max \left\{\mathbf{A}^{\top} \mathbf{D}^{-1} 1, \mathbf{D}^{-1} \mathbf{A} 1\right\} \leq(1+\alpha)\left[\mathbf{A}^{\top} \mathbf{D}^{-1} 1+\mathbf{D}^{-1} \mathbf{A} 1\right] .
$$

However, since $\mathbf{A} 1=d$ we have that

$$
\begin{aligned}
\left\|\mathbf{D}^{-1} e^{(0)}\right\|_{1} & \leq\left\|(1+\alpha) \mathbf{A}^{\top} \mathbf{D}^{-1} 1+(1+\alpha) \mathbf{D}^{-1} d-\mathbf{D}^{-1} d\right\|_{1} \\
& \leq(1+\alpha)\left\|\mathbf{A}^{\top} \mathbf{D}^{-1} 1\right\|_{1}+\alpha\|1\|_{1} \leq(1+2 \alpha)|V| .
\end{aligned}
$$

Where we used that $\left\|\mathbf{A}^{\top} \mathbf{D}^{-1} 1\right\|_{1}=1^{\top} \mathbf{A}^{\top} \mathbf{D}^{-1} 1=d^{\top} \mathbf{D}^{-1} 1=n$.

Lemma 8 (Formula for $e$ ). For $e, x \in \mathcal{R}_{>0}^{n}$ and $g \in \Delta^{n}$ let $v \stackrel{\text { def }}{=}\left(\mathbf{E}-g e^{\top}+\mathcal{L}\right) \mathbf{X} 1$ for directed Laplacian $\mathcal{L}=\mathbf{D}-\mathbf{A}^{\top}$. Then, $f \in \mathcal{R}^{n}$, the entry-wise minimal vector such that $(\mathbf{F}+\mathcal{L}) \mathbf{X}$ is $\alpha-R C D D$ is given by

$$
f=\alpha d+(1+\alpha) \max \left\{e-\mathbf{X}^{-1} v-\left(e^{\top} x\right) \mathbf{X}^{-1} g, 0\right\} .
$$

Proof. By Lemma 6 we know that $(\mathbf{F}+\mathcal{L}) \mathbf{X}$ is $\alpha$-CDD if and only if $f \geq \alpha$ d. Furthermore, $(\mathbf{F}+\mathcal{L}) \mathbf{X}$ is $\alpha$-RDD if and only if $(\mathbf{F}+\mathbf{D}) \mathbf{X} 1 \geq(1+\alpha) \mathbf{A}^{\top} \mathbf{X} 1$ which happens if and only if

$$
\begin{aligned}
f & \geq-d+(1+\alpha) \mathbf{X}^{-1} \mathbf{A}^{\top} \mathbf{X} 1 \\
& \geq-d+(1+\alpha) \mathbf{X}^{-1}\left[-v+\left(\mathbf{E}-g e^{\top}+\mathbf{D}\right) \mathbf{X} 1\right] \\
& =\alpha d+(1+\alpha)\left[-\mathbf{X}^{-1} v+e-\left(e^{\top} x\right) \mathbf{X}^{-1} g\right] .
\end{aligned}
$$

Taking the maximum of the two lower bounds on $f$ yields the result.

\subsubsection{Progress from Updating $e$}

Here we bound how much progress we make by updating $e$. We first give a general technical lemma, Lemma 9, and then in Lemma 10 we bound how $\left\|\mathbf{D}^{-1}\left(e^{(t)}-\alpha d\right)\right\|_{1}$ decreases in each iteration.

Lemma 9. For $a, b \in \mathcal{R}_{\geq 0}^{n}$ and $z \in \mathcal{R}_{>0}^{n}$ with $b_{i}=\max \left\{a_{i}-\frac{a^{\top} z}{\|a\|_{1}} \cdot \frac{a_{i}}{z_{i}}, 0\right\}$ we have $\|b\|_{1} \leq \frac{1}{2}\|a\|_{1}$. Proof. Let $T \stackrel{\text { def }}{=}\left\{i \in[n]: \frac{a^{\top} z}{\|a\|_{1}} \cdot \frac{a_{i}}{z_{i}} \leq a_{i}\right\}$. Then we have that

$$
\|a\|_{1}-\|b\|_{1}=\sum_{i \in[n]}\left(a_{i}-b_{i}\right)=\sum_{i \in T} \frac{a^{\top} z}{\|a\|_{1}} \cdot \frac{a_{i}}{z_{i}}+\sum_{i \in[n] \backslash T} a_{i} .
$$

We can bound $\|a\|_{1}^{2}$ trivially by

$$
\|a\|_{1}^{2}=\left(\sum_{i \in T} a_{i}+\sum_{i \in[n] \backslash T} a_{i}\right)^{2} \leq 2\left(\sum_{i \in T} a_{i}\right)^{2}+2\left(\sum_{i \in[n] \backslash T} a_{i}\right)^{2} .
$$

We can bound the first term by Cauchy-Schwarz

$$
\left(\sum_{i \in T} a_{i}\right)^{2}=\left(\sum_{i \in T} \frac{\sqrt{a_{i}}}{\sqrt{z_{i}}} \cdot \sqrt{z_{i} a_{i}}\right)^{2} \leq \sum_{i \in T} \frac{a_{i}}{z_{i}} \sum_{i \in T} a_{i} z_{i} \leq \sum_{i \in T} \frac{a_{i}}{z_{i}}\left(a^{\top} z\right) .
$$


and the second term trivially by

$$
\left(\sum_{i \in[n] \backslash T} a_{i}\right)^{2} \leq\left(\sum_{i \in[n] \backslash T} a_{i}\right) \sum_{i \in[n]} a_{i} \leq \sum_{i \in[n] \backslash T} a_{i}\|a\|_{1} .
$$

Combining (3.1), (3.2), (3.3), and (3.4) yields the result

$$
\frac{1}{2}\|a\|_{1} \leq \sum_{i \in T} \frac{a^{\top} z}{\|a\|_{1}} \cdot \frac{a_{i}}{z_{i}}+\sum_{i \in[n] \backslash T} a_{i}=\|a\|_{1}-\|b\|_{1} .
$$

Lemma 10 (Formula for $\left.e_{i}^{(t)}\right)$. For $e, x \in \mathcal{R}_{>0}^{n}$ and $g \stackrel{\text { def }}{=}\left\|\mathbf{D}^{-1} e\right\|_{1}^{-1} \mathbf{D}^{-1}$ e let $v \stackrel{\text { def }}{=}\left(\mathbf{E}-g e^{\top}+\mathcal{L}\right) \mathbf{X} 1$. Then setting $f \in \mathcal{R}^{n}$ to the entry-wise minimal vector such that $(\mathbf{F}+\mathcal{L}) \mathbf{X}$ is $\alpha$-RCDD gives:

$$
\left\|\mathbf{D}^{-1}(f-\alpha d)\right\|_{1} \leq \frac{1+\alpha}{2}\left\|\mathbf{D}^{-1}(e-\alpha d)\right\|_{1}+(1+\alpha)\left\|\mathbf{D}^{-1} \mathbf{X}^{-1} v\right\|_{1}+\left(\frac{1+\alpha}{2}\right) \frac{\alpha}{2} n .
$$

Proof. By Lemma 8 we know $f=\alpha d+(1+\alpha) \max \left\{e-\mathbf{X}^{-1} v-\left(e^{\top} x\right) \mathbf{X}^{-1} g, 0\right\}$. Consequently

$$
\begin{aligned}
\left\|\mathbf{D}^{-1}(f-\alpha d)\right\|_{1} & =(1+\alpha)\left\|\max \left\{\mathbf{D}^{-1} e-\mathbf{D}^{-1} \mathbf{X}^{-1} v-\left(e^{\top} x\right) \mathbf{D}^{-1} \mathbf{X}^{-1} g, 0\right\}\right\|_{1} \\
& \leq(1+\alpha)\left\|\mathbf{D}^{-1} \mathbf{X}^{-1} v\right\|_{1}+(1+\alpha)\left\|\max \left\{\mathbf{D}^{-1} e-\left(e^{\top} x\right) \mathbf{D}^{-1} \mathbf{X}^{-1} g, 0\right\}\right\|_{1} .
\end{aligned}
$$

Applying Lemma 9 with $a=\mathbf{D}^{-1} e$ and $z=\mathbf{D} x$ we see that

$$
\begin{aligned}
\left\|\max \left\{\mathbf{D}^{-1} e-\left(e^{\top} x\right) \mathbf{D}^{-1} \mathbf{X}^{-1} g, 0\right\}\right\|_{1} & =\left\|\max \left\{a-\frac{a^{\top} z}{\|a\|_{1}} \mathbf{Z}^{-1} a, 0\right\}\right\|_{1} \leq \frac{1}{2}\|a\|_{1} \\
& =\frac{1}{2}\left\|\mathbf{D}^{-1} e\right\|_{1} \leq \frac{1}{2}\left\|\mathbf{D}^{-1}(e-\alpha d)\right\|_{1}+\frac{\alpha}{2} n .
\end{aligned}
$$

Combining yields the result.

\subsubsection{Linear System Solving Accuracy}

Here we show how to deal with approximate computation.

Lemma 11. Let $\mathcal{L}=\mathbf{D}-\mathbf{A} \in \mathcal{R}^{n \times n}$ be a directed Laplacian and let $e, x \in \mathcal{R}_{>0}^{n}$ be such that $\mathbf{M}=(\mathbf{E}+\mathcal{L}) \mathbf{X}$ is $\alpha-R C D D$ for $\alpha \in\left(0, \frac{1}{2}\right)$. Let $g \stackrel{\text { def }}{=}\left\|\mathbf{D}^{-1} e\right\|_{1}^{-1} \mathbf{D}^{-1} e$ and let $z \in \mathcal{R}^{n}$ be an approximate solution to $\mathbf{M} z=g$ in the sense that for $z_{*} \stackrel{\text { def }}{=} \mathbf{M}^{-1} g$ we have

$$
\left\|z-z_{*}\right\|_{\operatorname{diag}(\mathbf{M})} \leq \frac{\epsilon}{\alpha}\|g\|_{\operatorname{diag}(\mathbf{M})^{-1}} .
$$

Furthermore, let $y$ be approximately $\mathbf{X} z$ in the sense that $\|\mathbf{D}(y-\mathbf{X} z)\|_{\infty} \leq \epsilon\left\|\mathbf{D}^{-1} e\right\|_{\infty}$. Then if

$$
\epsilon \leq \epsilon^{\prime} \cdot \frac{\alpha^{2}}{100} \cdot \frac{1}{\left\|\mathbf{D}^{-1} e\right\|_{1}} \cdot \frac{1}{\left(n+\left\|\mathbf{D}^{-1} e\right\|_{1}\right)} \cdot \frac{1}{\sqrt{n \cdot\left(1+\left\|\mathbf{D}^{-1} e\right\|_{\infty}\right) \kappa(\mathbf{D X})}}
$$

for $\epsilon^{\prime} \in(0,1)$ we have that $y>0$ and $\left\|\left(\mathbf{Y}_{*}\right)^{-1}\left(y-y_{*}\right)\right\|_{\infty} \leq \epsilon^{\prime}$ where $y_{*}=\mathbf{X M}^{-1} \mathbf{D}^{-1}$ e. Consequently, for $\mathcal{L}^{\prime}=\mathbf{E}-g e^{\top}+\mathcal{L}$ we have $\left\|\mathbf{Y}^{-1} \mathbf{D}^{-1} \mathcal{L}^{\prime} y\right\|_{1} \leq \epsilon^{\prime}$ and $\kappa(\mathbf{D Y}) \leq \frac{10}{\alpha^{2}}\left\|\mathbf{D}^{-1} e\right\|_{1}$. 
Proof. By Lemma 62 and the fact that $\|g\|_{1}=1$ by construction we have

$$
\operatorname{diag}(\mathbf{M})^{-1} g \leq z_{*} \leq \operatorname{diag}(\mathbf{M})^{-1} g+\frac{1}{\alpha} \operatorname{diag}(\mathbf{M})^{-1} 1 .
$$

Consequently, by (3.5)

$$
\begin{aligned}
& \left\|\mathbf{Z}_{*}^{-1}\left(z-z_{*}\right)\right\|_{\infty} \leq\left\|\operatorname{diag}(\mathbf{M}) \mathbf{G}^{-1}\left(z-z_{*}\right)\right\|_{2} \leq\left\|\mathbf{G}^{-1}\right\|_{2} \cdot \sqrt{\|\operatorname{diag}(\mathbf{M})\|_{2}} \cdot\left\|z-z_{*}\right\|_{\operatorname{diag}(\mathbf{M})} \\
& \leq\left\|\mathbf{G}^{-1}\right\|_{2} \cdot \sqrt{\|\operatorname{diag}(\mathbf{M})\|_{2}} \cdot \frac{\epsilon}{\alpha}\|g\|_{\operatorname{diag}(\mathbf{M})^{-1}} \\
& \leq \frac{\epsilon}{\alpha}\left\|\mathbf{G}^{-1}\right\|_{2} \cdot \sqrt{\|\operatorname{diag}(\mathbf{M})\|_{2} \cdot\left\|\operatorname{diag}(\mathbf{M})^{-1}\right\|_{2}} \cdot\|g\|_{2} \\
& \leq \frac{\epsilon}{\alpha}\left\|\mathbf{G}^{-1}\right\|_{2} \cdot \sqrt{\kappa(\operatorname{diag}(\mathbf{M}))} \cdot \sqrt{n}\|g\|_{\infty} \\
& \leq \frac{\epsilon}{\alpha} \kappa(\mathbf{G}) \sqrt{n \kappa(\operatorname{diag}(\mathbf{M}))}
\end{aligned}
$$

Now, $\kappa(\operatorname{diag}(\mathbf{M})) \leq\left(1+\left\|\mathbf{D}^{-1} e\right\|_{\infty}\right) \kappa(\mathbf{D X})$ and $\|\mathbf{G}\|_{2} \leq 1$. Furthermore, $\left\|\mathbf{G}^{-1}\right\|_{2} \leq \frac{1}{\alpha}\left\|\mathbf{D}^{-1} e\right\|_{1}$ since $\mathbf{E} \succeq \alpha \mathbf{D}$ by Lemma 6 and the fact that $\mathbf{M}$ is $\alpha$-RCDD. Consequently, $\kappa(\mathbf{G}) \leq \frac{1}{\alpha}\left\|\mathbf{D}^{-1} e\right\|_{1}$ and by our choice of $\epsilon$ we have

$$
\left\|\mathbf{Z}_{*}^{-1}\left(z-z_{*}\right)\right\|_{\infty} \leq \frac{\epsilon}{\alpha^{2}}\left\|\mathbf{D}^{-1} e\right\|_{1} \sqrt{n \cdot\left(1+\left\|\mathbf{D}^{-1} e\right\|_{\infty}\right) \kappa(\mathbf{D X})} \stackrel{\text { def }}{=} c_{1}
$$

This in turn implies that

$$
\begin{aligned}
\left\|\mathbf{Y}_{*}^{-1}\left(y-y_{*}\right)\right\|_{\infty} & =\left\|\mathbf{Z}_{*}^{-1} \mathbf{X}^{-1}\left(y-\mathbf{X} z_{*}\right)\right\|_{\infty} \leq\left\|\mathbf{Z}_{*}^{-1} \mathbf{X}^{-1}(y-\mathbf{X} z)\right\|_{\infty}+\left\|\mathbf{Z}_{*}^{-1} \mathbf{X}^{-1}\left(\mathbf{X} z_{*}-\mathbf{X} z\right)\right\|_{\infty} \\
& \leq\left\|\operatorname{diag}(\mathbf{M}) \mathbf{G}^{-1} \mathbf{X}^{-1}\left(y-\mathbf{X} z_{*}\right)\right\|_{\infty}+c_{1} \\
& \leq\left\|\operatorname{diag}(\mathbf{M}) \mathbf{G}^{-1} \mathbf{X}^{-1} \mathbf{D}^{-1}\right\|_{2} \cdot\left\|\mathbf{D}\left(y-\mathbf{X} z_{*}\right)\right\|_{\infty}+c_{1} \\
& =\left\|\mathbf{D}^{-1} e\right\|_{1} \cdot\left\|\mathbf{I}+\mathbf{D E}^{-1}\right\|_{2} \cdot \frac{\epsilon}{\alpha}+c_{1} \leq \frac{2}{\alpha}\left\|\mathbf{D}^{-1} e\right\|_{1} \cdot \frac{\epsilon}{\alpha}+c_{1} \\
& \leq \frac{3 \epsilon}{\alpha^{2}}\left\|\mathbf{D}^{-1} e\right\|_{1} \sqrt{n \cdot\left(1+\left\|\mathbf{D}^{-1} e\right\|_{\infty}\right) \kappa(\mathbf{D X})} \stackrel{\text { def }}{=} c_{2}
\end{aligned}
$$

where we used that $\left\|\mathbf{D E} \mathbf{E}^{-1}\right\|_{2} \leq \frac{1}{\alpha}$ by Lemma 6 and that $1 \leq \frac{1}{\alpha}$ by assumption that $\alpha<\frac{1}{2}$. Consequently, we see that $y$ is within a small multiplicative constant of $y_{*}$, i.e. $\frac{1}{2} y_{*} \leq\left(1-c_{2}\right) y_{*} \leq$ $y \leq\left(1+c_{2}\right) y_{*} \leq 1 y_{*}$ since by our choice of $\epsilon c_{2} \leq \frac{1}{2}$. Therefore, applying Lemma 5 we know that $\mathcal{L}^{\prime} y_{*}=0$ and

$$
\begin{aligned}
\left\|\mathbf{Y}^{-1} \mathbf{D}^{-1} \mathcal{L}^{\prime} y\right\|_{1} & =\left\|\mathbf{Y}^{-1} \mathbf{D}^{-1} \mathcal{L}^{\prime}\left(y-y_{*}\right)\right\|_{1} \leq 2\left\|\mathbf{Y}_{*}^{-1} \mathbf{D}^{-1} \mathcal{L}^{\prime} \mathbf{Y}_{*} \mathbf{Y}_{*}^{-1}\left(y-y_{*}\right)\right\|_{1} \\
& \leq 4\left\|\operatorname{diag}\left(\mathbf{Y}_{*}^{-1} \mathbf{D}^{-1} \mathcal{L}^{\prime} \mathbf{Y}_{*}\right)\right\|_{1}\left\|\mathbf{Y}_{*}^{-1}\left(y-y_{*}\right)\right\|_{\infty} \\
& \leq 4\left\|\operatorname{diag}\left(\mathbf{I}+\mathbf{D}^{-1} \mathbf{E}\right)\right\|_{1} \cdot c_{2} \leq 4\left(n+\left\|\mathbf{D}^{-1} e\right\|_{1}\right) \cdot c_{2} \\
& \leq \frac{12 \epsilon}{\alpha^{2}}\left\|\mathbf{D}^{-1} e\right\|_{1}\left(n+\left\|\mathbf{D}^{-1} e\right\|_{1}\right) \sqrt{n \cdot\left(1+\left\|\mathbf{D}^{-1} e\right\|_{\infty}\right) \kappa(\mathbf{D X})} .
\end{aligned}
$$

Where we used that $\mathcal{L}^{\prime} \mathbf{Y}_{*}$ is a Eulerian Laplacian so $\mathbf{Y}_{*}^{-1} \mathbf{D}^{-1} \mathcal{L}^{\prime} \mathbf{Y}_{*}$ is $\operatorname{RDD}$ and we can apply Lemma 61 . Finally, we note that by (3.6)

$$
\begin{aligned}
\kappa\left(\mathbf{D Y}_{*}\right) & =\left\|\mathbf{D} \mathbf{Z}_{*} \mathbf{X}\right\|_{2} \cdot\left\|\mathbf{D}^{-1} \mathbf{Z}_{*}^{-1} \mathbf{X}^{-1}\right\|_{2} \\
& \leq\left\|\left(\operatorname{diag}(\mathbf{M})^{-1} \mathbf{G}+\frac{1}{\alpha} \operatorname{diag}(\mathbf{M})^{-1}\right) \mathbf{D X}\right\|_{2} \cdot\left\|\mathbf{D}^{-1} \mathbf{X}^{-1} \operatorname{diag}(\mathbf{M}) \mathbf{G}^{-1}\right\|_{2} \\
& \leq\left\|\left(\mathbf{D}^{-1} \mathbf{E}+\mathbf{I}\right)^{-1}\left(\mathbf{G}+\alpha^{-1} \mathbf{I}\right)\right\|_{2} \cdot\left\|\left(\mathbf{D}^{-1} \mathbf{E}+\mathbf{I}\right) \mathbf{G}^{-1}\right\|_{2} \\
& \leq \frac{2}{\alpha} \cdot\left\|\mathbf{D}^{-1} e\right\|_{1}\left\|\left(\mathbf{I}+\mathbf{D E}^{-1}\right)\right\|_{2} \leq \frac{4}{\alpha^{2}}\left\|\mathbf{D}^{-1} e\right\|_{1}
\end{aligned}
$$

Where we used $\mathbf{G} \preceq \mathbf{I} \preceq \frac{1}{\alpha} \mathbf{I}$ and $\mathbf{D}^{-1} \mathbf{E} \succeq \alpha \mathbf{I}$. Our bound on $\left\|\mathbf{Y}_{*}^{-1}\left(y-y_{*}\right)\right\|$ completes the result. 


\subsubsection{Putting it All Together}

Here we show how to put together the results of the previous subsections to prove Theorem 2.

Proof of Theorem 2. The running time is immediate from the definition of the algorithm and the fact that for exact $x^{(t)}$ computation it is the case that $v^{(t)}=0$ by Lemma 5 . What remains is to prove that $(3 \alpha|V| \cdot \mathbf{D}+\mathcal{L}) \mathbf{D}^{-1} \mathbf{S}$ is an $\alpha$-RCDD. Now, by Lemma 6 and Lemma 7 we know that

$$
\left\|\mathbf{D}^{-1}\left(e^{(0)}-\alpha d\right)\right\|_{1} \leq\left\|\mathbf{D}^{-1} e^{(0)}\right\|_{1}-\alpha\left\|\mathbf{D}^{-1} d\right\|_{1} \leq(1+\alpha) \cdot n \leq 2 n
$$

and clearly $\kappa\left(\mathbf{D X}^{(0)}\right)=1$. Now suppose that for some $t \geq 0$ we have that

$$
\left\|\mathbf{D}^{-1}\left(e^{(t)}-\alpha d\right)\right\|_{1} \leq \max \left\{\left(\frac{7}{8}\right)^{t} 2 n, 2 \alpha n\right\}
$$

and $\kappa\left(\mathbf{D} \mathbf{X}^{(t)}\right) \leq \frac{20}{\alpha^{2}} n$. Then since this implies $\left\|\mathbf{D}^{-1} e^{(t)}\right\|_{1} \leq 2 n$ by Lemma 11 we have that for any absolute constant $\epsilon^{\prime}$ it is the case that $x^{(t+1)}>0$ and $\left\|\left[\mathbf{X}^{(t+1)}\right]^{-1} \mathbf{D}^{-1} \mathcal{L}^{(t)} x^{(t+1)}\right\|_{1} \leq \epsilon^{\prime}$ and $\kappa\left(\mathbf{D X}^{(t+1)}\right) \leq \frac{10}{\alpha^{2}}\left\|\mathbf{D}^{-1} e^{(t)}\right\|_{1} \leq \frac{20}{\alpha^{2}} n$. Furthermore, this implies by Lemma 10 that for $\epsilon^{\prime} \leq \frac{1}{24}$

$$
\begin{aligned}
\left\|\mathbf{D}^{-1}\left(e^{(t+1)}-\alpha d\right)\right\|_{1} & \leq \frac{1+\alpha}{2}\left\|\mathbf{D}^{-1}\left(e^{(t)}-\alpha d\right)\right\|_{1}+(1+\alpha) \epsilon^{\prime}+\left(\frac{1+\alpha}{2}\right) \frac{\alpha}{2} n \\
& \leq \frac{3}{4}\left\|\mathbf{D}^{-1}\left(e^{(t)}-\alpha d\right)\right\|_{1}+\frac{1}{4} \alpha n \leq \max \left\{\left(\frac{7}{8}\right)^{t+1} 2 n, 2 \alpha n\right\}
\end{aligned}
$$

where in the last line we used that if $\left\|\mathbf{D}^{-1}\left(e^{(t)}-\alpha d\right)\right\|_{1} \geq 2 \alpha n$, then decreasing it by $\frac{3}{4}=\left(1-\frac{1}{4}\right)$ is the same as decreasing by $\frac{7}{8}=\left(1-\frac{1}{8}\right)$ and then subtracting $\frac{1}{8}$ fraction is subtracting at least $\frac{1}{4} \alpha n$, which cancels the additive term.

Consequently, by induction we have that $\left\|\mathbf{D}^{-1}\left(e^{(t)}-\alpha d\right)\right\|_{1} \leq \max \left\{\left(\frac{7}{8}\right)^{t} 2 n, 2 \alpha n\right\}$ for all $t$. Therefore, for $k=8 \ln \alpha^{-1}$ we have

$$
\left(\frac{7}{8}\right)^{t} 2 n=\left(1-\frac{1}{8}\right)^{t} 2 n \leq e^{-\frac{t}{8}} 2 n \leq 2 n \alpha
$$

and $\left\|\mathbf{D}^{-1}\left(e^{(k+1)}-\alpha d\right)\right\|_{\infty} \leq\left\|\mathbf{D}^{-1}\left(e^{(k+1)}-\alpha d\right)\right\|_{1} \leq 2 n \alpha$; therefore $e^{(t)} \leq 3 \alpha n \cdot d$. Furthermore, since $\left(\mathbf{E}^{(k+1)}+\mathcal{L}\right) \mathbf{X}^{(k+1)}$ is $\alpha$-RCDD by construction this implies that $(3 n \alpha \mathbf{D}+\mathcal{L}) \mathbf{X}^{(k)}$ is $\alpha$-RCDD, yielding the result.

\section{Eulerian Laplacian Solver}

Throughout this section, let $\mathcal{L}$ denote an Eulerian directed Laplacian with $n$ vertices and $m$ edges, and let $\mathbf{U}$ denote the associated undirected Laplacian: $\mathbf{U} \stackrel{\text { def }}{=} \frac{1}{2}\left(\mathcal{L}+\mathcal{L}^{\top}\right)$. Let $\mathbf{B}$ denote a weighted edge-vertex incidence matrix for $\mathbf{U}$, so that $\mathbf{U}=\mathbf{B}^{\top} \mathbf{B}$. We define $\mathcal{T}_{\text {solve }} \stackrel{\text { def }}{=}\left(n m^{3 / 4}+n^{2 / 3} m\right)(\log n)^{3}$ to simplify the statements of our runtime bounds.

The goal of this section is to prove the following, Theorem 12, showing that we can efficiently solve linear systems in $\mathcal{L}$. Note that we make no attempt to minimize the number of logarithmic factors in this presentation (including e.g. in parameter balancing); however, we suspect that with careful use of the recent results [30] and [31] the $\log n$ factors can all be eliminated. 
Theorem 12. Let $b$ be an $n$-dimensional vector in the image of $\mathcal{L}$, and let $x$ be the solution to $\mathcal{L} x=b$. Then for any $0<\epsilon \leq \frac{1}{2}$, one can compute in $O\left(\mathcal{T}_{\text {solve }} \log (1 / \epsilon)\right)$ time, a vector $x^{\prime}$ which with high probability is an approximate solution to the linear system in that $\left\|x^{\prime}-x\right\|_{\mathbf{U}} \leq \epsilon\|b\|_{\mathbf{U}^{+}}$.

Our proof of Theorem 12 is crucially based on the symmetric matrix $\mathbf{X} \stackrel{\text { def }}{=} \mathcal{L}^{\top} \mathbf{U}^{+} \mathcal{L}$. We begin by noting that $\mathbf{X}$ is somewhat well approximated by $\mathbf{U}$ :

Lemma 13. $\mathbf{X} \succeq \mathbf{U}$, while $\operatorname{tr}\left(\mathbf{X} \mathbf{U}^{+}\right)=\operatorname{tr}\left(\mathcal{L}^{\top} \mathbf{U}^{+} \mathcal{L} \mathbf{U}^{+}\right) \leq 2(n-1)^{2}$.

Proof. We first show that $\mathbf{U} \preceq \mathbf{X}$. Let $\mathbf{V}$ denote the antisymmetric matrix $\frac{1}{2}\left(\mathcal{L}-\mathcal{L}^{\top}\right)$ so that $\mathcal{L}=\mathbf{U}+V$. With this notation we have

$$
\begin{aligned}
\mathbf{X} & =(\mathbf{U}+\mathbf{V})^{\top} \mathbf{U}^{+}(\mathbf{U}+\mathbf{V})=(\mathbf{U}-\mathbf{V}) \mathbf{U}^{+}(\mathbf{U}+\mathbf{V}) \\
& =\mathbf{U}-\mathbf{V}+\mathbf{V}-\mathbf{V} \mathbf{U}^{+} \mathbf{V}=\mathbf{U}+\mathbf{V}^{\top} \mathbf{U}^{+} \mathbf{V} \succeq \mathbf{U}
\end{aligned}
$$

Next, for the trace bound, we first note as $\mathcal{L}$ is an Eulerian Laplacian it can be decomposed as a sum over simple cycles (multiplied by a scalar weight) $\mathcal{L}_{i}$, i.e.

$$
\mathcal{L}=\sum_{i} \mathcal{L}_{i} \quad \text { and } \quad \mathbf{U}=\sum_{i} \mathbf{U}_{i}
$$

where $\mathbf{U}_{i} \stackrel{\text { def }}{=} \frac{1}{2}\left(\mathcal{L}_{i}+\mathcal{L}_{i}^{\top}\right)$

Now we claim $\mathbf{X} \preceq \sum_{i} \mathcal{L}_{i}^{\top} \mathbf{U}_{i}^{+} \mathcal{L}_{i}$. This can be shown by simply directly bounding the quadratic form for each input, showing that

$$
v^{\top} \mathbf{X} v \leq \sum_{i}\left(\mathcal{L}_{i} v\right)^{\top} \mathbf{U}_{i}^{+}\left(\mathcal{L}_{i} v\right)
$$

This in turn follows from the general statement that for any two vectors $u$ and $v$, and positive definite matrices $\mathbf{A}$ and $\mathbf{B}$,

$$
(u+v)^{\top}(\mathbf{A}+\mathbf{B})^{-1}(u+v) \leq u^{\top} \mathbf{A}^{-1} u+v^{\top} \mathbf{B}^{-1} v .
$$

Consequently, our claim $\mathbf{X} \preceq \sum_{i} \mathcal{L}_{i}^{\top} \mathbf{U}_{i}^{+} \mathcal{L}_{i}$ holds and therefore $\operatorname{tr}\left(\mathbf{X} \mathbf{U}^{+}\right) \leq \sum_{i} \operatorname{tr}\left(\mathcal{L}_{i}^{\top} \mathbf{U}_{i}^{+} \mathcal{L}_{i} \mathbf{U}^{+}\right)$.

Next, we note that if $\mathcal{L}_{i}$ is a simple cycle of length $k_{i}$ and weight $w_{i}$ through the vertices $V_{i} \subseteq V$, then $\mathcal{L}_{i}^{\top} \mathbf{U}_{i}^{+} \mathcal{L}_{i}$ is precisely the clique on those $k_{i}$ vertices, multiplied by $\frac{2 w_{i}}{k_{i}}$. This can be verified, for instance, using an eigenvector decomposition (the Fourier vectors simultaneously diagonalize $\mathcal{L}_{i}$, $\mathcal{L}_{i}^{\top}$, and $\left.\mathbf{U}_{i}\right)$. We then can express $\operatorname{tr}\left(\mathcal{L}_{i}^{\top} \mathbf{U}_{i}^{+} \mathcal{L}_{i} \mathbf{U}^{+}\right)$as $\frac{2 w_{i}}{k_{i}}$ times the sum over all pairs of vertices $u, v \in V_{i}$ of the effective resistance between $u$ and $v$ in $\mathbf{U}$. Effective resistances form a metric; since any two vertices in $V_{i}$ are connected by some path through the cycle, their effective resistance must always be at most the sum of the effective resistances between the edges in the cycle, which in turn is $\frac{2}{w_{i}} \operatorname{tr}\left(\mathbf{U}_{i} \mathbf{U}^{+}\right)$. With $\frac{k_{i}\left(k_{i}-1\right)}{2}$ pairs of vertices, we have

$$
\operatorname{tr}\left(\mathcal{L}_{i}^{\top} \mathbf{U}_{i}^{+} \mathcal{L}_{i} \mathbf{U}^{+}\right) \leq 2\left(k_{i}-1\right) \operatorname{tr}\left(\mathbf{U}_{i} \mathbf{U}^{+}\right) \leq 2(n-1) \operatorname{tr}\left(\mathbf{U}_{i} \mathbf{U}^{+}\right)
$$

and

$$
\sum_{i} \operatorname{tr}\left(\mathcal{L}_{i}^{\top} \mathbf{U}_{i}^{+} \mathcal{L}_{i} \mathbf{U}^{+}\right) \leq 2(n-1) \sum_{i} \operatorname{tr}\left(\mathbf{U}_{i} \mathbf{U}^{+}\right) \leq 2(n-1) \operatorname{tr}\left(\mathbf{U U}^{+}\right)=2(n-1)^{2} .
$$


Lemma 13 immediately suggests our approach: we essentially solve systems in $\mathcal{L}$ by solving systems in $\mathbf{X}$ (technically, we use a slightly different matrix $\widetilde{\mathbf{X}}$ which we define later), and use preconditioning schemes for symmetric positive definite matrices based on the structure of $\mathbf{X}$ and U.

A simple preconditioning strategy would be to directly use $\mathbf{U}$ as a preconditioner for $\mathbf{X}$ in an algorithm like preconditioned Chebyshev iteration. This depends on the relative condition number of $\mathbf{U}$ and $\mathbf{X}$; the relative condition number of two symmetric positive semidefinite matrices $\mathbf{A}$ and $\mathbf{B}$ is defined to be infinite if they don't have the same null space, and otherwise to be $\frac{\lambda_{\max }\left(\mathbf{A B}^{+}\right)}{\lambda_{\min }\left(\mathbf{A B}^{+}\right)}$. Since the relative condition number of $\mathbf{X}$ and $\mathbf{U}$ is at most $O\left(n^{2}\right)$ by Lemma 13 and iterative algorithms like preconditioned Chebyshev iteration have an iteration count proportional to the square root of the relative condition number, this would require about $n$ iterations for a total runtime of about $\mathrm{nm}$ (using fast Laplacian solvers to solve systems in $\mathbf{U}$ ).

One might hope to improve this by plugging the trace bound into a result from [56], which says that if one uses preconditioned conjugate gradient in exact arithmetic, the iteration count scales only with the cube root of the trace. That would give a runtime of about $n^{2 / 3} m$. However, it is unknown how to obtain this result without using exact arithmetic (or a polynomial number of bits of precision). Since we are aiming for algorithms which only need a logarithmic number of bits of precision (see Appendix A) it is unclear how to leverage preconditioned conjugate gradient directly to improve the running time and this remains an interesting open question.

Instead use a technique similar to the method of transmogrification used in Laplacian solvers, in particular [28]. However, our method deviates from much of this previous work in that it is nonrecursive and uses only the Woodbury matrix identity (instead of partial Cholesky factorization) to solve the preconditioner. This allows us to obtain a preconditioner with a better relative condition number than $\mathbf{U}$ itself, which can still be applied nearly as efficiently. First, we state the "matrix Chernoff bound" that we will use to construct the preconditioner:

Lemma 14. Let $\epsilon, \delta \in(0,1 / 2)$. Let $\mathbf{M}=\sum_{i} \mathbf{M}_{i}$ be the sum of independent random symmetric $n$-dimensional matrices $\mathbf{M}_{i}$, where each $\mathbf{M}_{i}$ is positive definite and satisfies $\left\|\mathbf{M}_{i}\right\|_{2} \leq \frac{\epsilon^{2}}{3 \log (2 n / \delta)}$ with probability 1. Furthermore, let $\mathrm{E}[\mathbf{M}] \preceq \mathbf{I}$. Then $\|\mathbf{M}-\mathrm{E}[\mathbf{M}]\| \leq \epsilon$ with probability at least $1-\delta$.

Proof. Apply Theorem 1.4 ("matrix Bernstein") from [58] to $\sum_{i}\left(\mathbf{M}_{i}-\mathrm{E}\left[\mathbf{M}_{i}\right]\right)$, and let $R^{\prime}=\frac{\epsilon^{2}}{3 \log (2 n / \delta)}$. We have unconditionally, for all $i,\left\|\mathbf{M}_{i}-\mathrm{E}\left[\mathbf{M}_{i}\right]\right\| \leq\left\|\mathbf{M}_{i}\right\|+\left\|\mathrm{E}\left[\mathbf{M}_{i}\right]\right\| \leq 2 R^{\prime}$. Furthermore, we have

$$
\sum_{i} \mathrm{E}\left[\left(\mathbf{M}_{i}-\mathrm{E}\left[\mathbf{M}_{i}\right]\right)^{2}\right]=\sum_{i}\left(\mathrm{E}\left[\mathbf{M}_{i}^{2}\right]-\left(\mathrm{E}\left[\mathbf{M}_{i}\right]\right)^{2}\right) \preceq \sum_{i} \mathrm{E}\left[\mathbf{M}_{i}^{2}\right] \preceq R^{\prime} \sum_{i} \mathrm{E}\left[\mathbf{M}_{i}\right] \preceq R^{\prime} \mathbf{I} .
$$

We thus have $R=2 R^{\prime}$ and $\sigma^{2} \leq R^{\prime}$ in that theorem statement. The probability that the max eigenvalue of $\mathbf{M}-\mathrm{E}[\mathbf{M}]$ is greater than $\epsilon$ is then at most

$$
\begin{aligned}
n \exp \left(-\frac{\epsilon^{2} / 2}{R^{\prime}+2 R^{\prime} \epsilon / 3}\right) & =n \exp \left(-\frac{\epsilon^{2}}{2 R^{\prime}(1+2 \epsilon / 3)}\right) \leq n \exp \left(-\frac{\epsilon^{2}}{3 R^{\prime}}\right) \\
& \leq n \exp (-\log (2 n / \delta))=\frac{\delta}{2} .
\end{aligned}
$$

Applying the same argument to the negation $\mathrm{E}[\mathbf{M}]-\mathbf{M}$ and union bounding gives the result.

For notational convenience (to deal with inexact Laplacian system solvers), for the next statements, we let $\widetilde{\mathbf{U}}$ be any symmetric matrix satisfying $\frac{1}{2} \mathbf{U} \preceq \widetilde{\mathbf{U}} \preceq \mathbf{U}$, and let $\widetilde{\mathbf{X}} \stackrel{\text { def }}{=} \mathcal{L}^{\top} \widetilde{\mathbf{U}}^{+} \mathcal{L}$. The next statement gives us a method to construct preconditioners for $\mathbf{X}$; it is inspired by the "ultrasparsifiers" or "incremental sparsifiers" from [28]. 
Lemma 15. For any $k \in\left[0, n^{2}\right]$, there exists a set of nonnegative weights $w_{i}$, with at most $O\left(\frac{n^{2} \log n}{k}\right)$ of them nonzero, such that

$$
\frac{1}{2}\left(\widetilde{\mathbf{U}}+\frac{1}{k} \widetilde{\mathbf{X}}\right) \preceq \widetilde{\mathbf{U}}+\mathcal{L}^{\top} \widetilde{\mathbf{U}}^{+} \mathbf{B}^{\top} \mathbf{W B} \widetilde{\mathbf{U}}^{+} \mathcal{L} \preceq 2\left(\widetilde{\mathbf{U}}+\frac{1}{k} \widetilde{\mathbf{X}}\right)
$$

Furthermore, if $\widetilde{\mathbf{U}}^{+}$can be applied to a vector in $O(m \log n)$ time, such weights can be computed in time $O\left(m(\log n)^{2}\right)$ with high probability. Note that no access to $\widetilde{\mathbf{U}}$ itself is needed; only its pseudoinverse.

Proof. Define $l_{i} \stackrel{\text { def }}{=}\left\|\mathcal{L}^{\top} \widetilde{\mathbf{U}}^{+} b_{i}\right\|_{\widetilde{\mathbf{U}}^{+}}$where $b_{i}$ is the $i$ th row of $\mathbf{B}$ for all $i \in[n]$. Then there exists some universal constant $C$ (depending on the exponent in the "high probability") such that if $p_{i} \geq C l_{i} \log n$, we can independently pick $\frac{\sum_{i} p_{i}}{k}$ rows of $\mathbf{B}$, with replacement, with probability of picking row $i$ each time being $\frac{p_{i}}{\sum_{i} p_{i}}$, then add $\frac{1}{p_{i}}$ to $w_{i}$ each time $i$ is picked. Here, we define $\mathbf{V}=\widetilde{\mathbf{U}}+\frac{1}{k} \widetilde{\mathbf{X}}$ and $\widetilde{\mathbf{V}}=\widetilde{\mathbf{U}}+\mathcal{L}^{\top} \widetilde{\mathbf{U}}^{+} \mathbf{B}^{\top} \mathbf{W B} \widetilde{\mathbf{U}}^{+} \mathcal{L}$. Then we apply Lemma 14 to $\mathbf{V}^{-\frac{1}{2}}(\widetilde{\mathbf{V}}-\widetilde{\mathbf{U}}) \mathbf{V}^{-\frac{1}{2}}$, which is a sum of independent random matrices, with expectation $\preceq \mathbf{I}$, with each term having spectral norm at most $\frac{1}{C \log n}$. If $C$ is set sufficiently large this guarantees the desired result.

We have

$$
\begin{aligned}
\sum_{i \in[n]} l_{i} & =\sum_{i \in[n]} b_{i}^{\top} \widetilde{\mathbf{U}}^{+} \mathcal{L} \widetilde{\mathbf{U}}^{+} \mathcal{L}^{\top} \widetilde{\mathbf{U}}^{+} b_{i}=\sum_{i \in[n]} \operatorname{tr}\left(\mathcal{L}^{\top} \widetilde{\mathbf{U}}^{+} b_{i} b_{i}^{\top} \widetilde{\mathbf{U}}^{+} \mathcal{L} \widetilde{\mathbf{U}}^{+}\right) \leq \operatorname{tr}\left(\mathcal{L}^{\top}\left(4 \mathbf{U}^{+}\right) \mathcal{L}\left(2 \mathbf{U}^{+}\right)\right) \\
& =8 \operatorname{tr}\left(\mathbf{X} \mathbf{U}^{+}\right)=O\left(n^{2}\right) .
\end{aligned}
$$

where the last line used Lemma 13. Thus, if we can compute a constant-factor approximation to the $l_{i}$, we can obtain $p_{i} \geq C l_{i} \log (n)$ with $\sum_{i} p_{i}=O\left(n^{2} \log n\right)$, and the random sample will be of size $O\left(k^{-1} n^{2} \log n\right)$ as desired. Next, we note that $l_{i}$ is within a factor of 2 of $\left\|\mathbf{B} \widetilde{\mathbf{U}}^{+} \mathcal{L} \widetilde{\mathbf{U}}^{+} b_{i}\right\|_{2}$, since $\widetilde{\mathbf{U}}^{+} \mathbf{B}^{\top} \mathbf{B} \widetilde{\mathbf{U}}^{+}=\mathbf{U}^{+} \mathbf{U} \mathbf{U}^{+}$is within a factor of two of $\widetilde{\mathbf{U}}^{+}=\widetilde{\mathbf{U}}^{+} \widetilde{\mathbf{U}}^{+}$. Thus a constant factor approximation to the latter expression is sufficient. Finally, we note that we can compute all of these constant factor approximations using Johnson-Lindenstrauss (analogous to the construction in [54]). In particular, we may let $\boldsymbol{\Pi}$ be a Gaussian random matrix with $r=O(\log n)$ rows and $m$ columns, rescaled by $\frac{1}{\sqrt{r}}$. Then the Johnson-Lindenstrauss lemma implies that with high probability, $\left\|\mathbf{\Pi B} \widetilde{\mathbf{U}}^{+} \mathcal{L} \widetilde{\mathbf{U}}^{+} b_{i}\right\|_{2}$ will be within a constant factor of $\left\|\mathbf{B} \widetilde{\mathbf{U}}^{+} \mathcal{L} \widetilde{\mathbf{U}}^{+} b_{i}\right\|_{2}$ for all $i$. We may compute each row of $\boldsymbol{\Pi B} \widetilde{\mathbf{U}}^{+} \mathcal{L} \widetilde{\mathbf{U}}^{+}$in $O(m \log n)$ time by successively multiplying the rows of $\boldsymbol{\Pi}$ by $m$-sparse matrices and $\widetilde{\mathbf{U}}^{+}$, each of which can be done in $O(m \log n)$ time. Thus computing it for all rows takes time $O\left(m(\log n)^{2}\right)$. Finally, since $\mathbf{\Pi B} \widetilde{\mathbf{U}}^{+} \mathcal{L} \widetilde{\mathbf{U}}^{+}$has $O(\log n)$ rows and $b_{i}$ is 2 -sparse, we can compute each $\Pi \mathbf{\Pi B} \widetilde{\mathbf{U}}^{+} \mathcal{L} \widetilde{\mathbf{U}}^{+} b_{i}$ in $O(\log n)$ time, taking $O(m \log n)$ time in total.

Lemma 15 says that for large $k$, we can approximate $\widetilde{U}+\frac{1}{k} \widetilde{X}$ with a low-rank update to $\widetilde{\mathbf{U}}$, i.e. rank $O\left(k^{-1} n^{2} \log n\right)$ to be precise. In the next lemma we show that with a little precomputation, we can apply the pseudoinverse of this approximation efficiently.

Lemma 16. Consider the matrix $\mathbf{Z}=\widetilde{\mathbf{U}}+\mathcal{L}^{\top} \widetilde{\mathbf{U}}^{+} \mathbf{B}^{\top} \mathbf{W B} \widetilde{\mathbf{U}}^{+} \mathcal{L}$. If $\widetilde{\mathbf{U}}^{+}$can be applied to a vector in time $T$, and $\mathbf{W}$ has $r$ non-zero entries, then after precomputation taking $O\left((m+T) r+r^{3}\right)$ time, we can apply $\mathbf{Z}^{+}$to a vector in $O\left(m+T+r^{2}\right)$ time.

Proof. We define $\mathbf{R}$ to be the restriction of $\mathbf{B} \widetilde{\mathbf{U}}^{+} \mathcal{L}$ to the rows where $\mathbf{W}$ is nonzero, so that $\mathbf{Z}=\widetilde{\mathbf{U}}+\mathbf{R}^{\top} \mathbf{W}_{S} \mathbf{R}$ (where $\mathbf{W}_{S}$ is $\mathbf{W}$ similarly restricted). Then we can apply the Woodbury matrix identity to get that

$$
\mathbf{Z}^{+}=\widetilde{\mathbf{U}}^{+}-\widetilde{\mathbf{U}}^{+} \mathbf{R}^{\top}\left(\mathbf{W}_{S}^{-1}+\mathbf{R} \widetilde{\mathbf{U}}^{+} \mathbf{R}^{\top}\right)^{-1} \mathbf{R} \widetilde{\mathbf{U}}^{+} .
$$


First, we define the precomputation stage, which computes the entries of the matrix $\mathbf{M}=$ $\left(\mathbf{W}_{S}^{-1}+\mathbf{R} \widetilde{\mathbf{U}}^{+} \mathbf{R}^{\top}\right)^{-1}$. This has two logical steps: computing the entries of $\mathbf{R} \widetilde{\mathbf{U}}^{+} \mathbf{R}^{\top}$ and then computing the matrix inverse. We compute $\mathbf{R} \widetilde{\mathbf{U}}^{+} \mathbf{R}^{\top}$ column by column, simply applying $\mathbf{R}^{\top}, \widetilde{\mathbf{U}}^{+}$, and $\mathbf{R}$ in succession to each of the standard basis vectors. The applications of $\mathbf{R}$ and $\mathbf{R}^{\top}$ are done by successively applying $\mathbf{B}, \widetilde{\mathbf{U}}^{+}, \mathcal{L}$, and their transposes; each of these is either an $O(m)$-sparse matrix or one which we are promised can be applied in time $T$. Thus, each of these columns can be computed in time at most $O(m+T)$; getting all $r$ columns then takes $O((m+T) r)$ time. Adding this to $\mathbf{W}_{S}^{-1}$ (which is diagonal) and then inverting the $r \times r$ matrix takes time at most $O\left(r^{3}\right)$ even using a naive algorithm such as Gaussian elimination (which already suffices to achieve our desired result).

Now, once this $\mathbf{M}$ has been precomputed, we can apply the operator $\mathbf{Z}^{+}$to a vector just by applying $\widetilde{\mathbf{U}}^{+}$and applying $\widetilde{\mathbf{U}}^{+} \mathbf{R}^{\top} \mathbf{M R} \widetilde{\mathbf{U}}^{+}$by applying the individual factors successively. Applying $\mathbf{M}$ costs at most $O\left(r^{2}\right)$ since that is the size of the matrix, while the other applications take $O(m+T)$; thus the total time to apply is $O\left(m+T+r^{2}\right)$.

Lemma 17. If $\widetilde{\mathbf{U}}^{+}$can be applied to a vector in $O(m \log n)$ time, we can, with high probability, apply a linear operator $\mathbf{Y}^{+}$satisfying $(1-\epsilon) \widetilde{\mathbf{X}}^{+} \preceq \mathbf{Y}^{+} \preceq(1+\epsilon) \widetilde{\mathbf{X}}^{+}$in $O\left(\left(n m^{3 / 4}+\right.\right.$ $\left.\left.n^{2 / 3} m\right)(\log n)^{3} \log (1 / \epsilon)\right)=O\left(\mathcal{T}_{\text {solve }} \log (1 / \epsilon)\right)$ time.

Proof. Invoke 15 for $k=\max \left(n^{2} / \sqrt{m}, n^{4 / 3}\right)$, and define $\mathbf{Z}=\widetilde{\mathbf{U}}+\mathcal{L}^{\top} \widetilde{\mathbf{U}}^{+} \mathbf{B}^{\top} \mathbf{W B} \widetilde{\mathbf{U}}^{+} \mathcal{L}$.

The number of nonzero entries in $\mathbf{W}$ will then be at $\operatorname{most} O\left(\min \left(\sqrt{m}, n^{2 / 3}\right) \log n\right)$. Now, apply 16. The precomputation takes time $O\left((m \log n) r+r^{3}\right) \leq O\left(n^{2 / 3} m(\log n)^{2}+\min \left(m^{3 / 2}, n^{2}\right)(\log n)^{3}\right)$. Since $\min \left(m^{3 / 2}, n^{2}\right) \leq n m^{3 / 4}+n^{2 / 3} m$, this is $O\left(\left(n m^{3 / 4}+n^{2 / 3} m\right)(\log n)^{3}\right.$. The time per application is at most $O\left(m+m \log n+(\sqrt{m} \log n)^{2}\right)=O\left(m(\log n)^{2}\right)$.

Now, we use $\mathbf{Z}^{+}$as a preconditioner in an iterative method: preconditioned Chebyshev iteration. Because the relative condition number between $\mathbf{Z}$ and $\widetilde{\mathbf{X}}$ is $O(k)$, the number of iterations required is $O(\sqrt{k} \log (1 / \epsilon))=O\left(\left(n / m^{1 / 4}+n^{2 / 3}\right) \log (1 / \epsilon)\right)$. Each iteration requires one application of $\widetilde{\mathbf{X}}$ and one application of $\mathbf{Z}^{+}$to vectors, each of which takes $O\left(m(\log n)^{2}\right)$ time. The total running time of this solve phase is then at most $O\left(\left(n m^{3 / 4}+n^{2 / 3} m\right)(\log n)^{2} \log (1 / \epsilon)\right)$.

Now, we turn Lemma 17 into a solver for $\mathcal{L}$.

Lemma 18. Let $b$ be an $n$-dimensional vector in the image of $\mathcal{L}$, and let $x$ be the solution to $\mathcal{L} x=b$. Then for any $0<\epsilon \leq \frac{1}{2}$, one can compute in $O\left(\mathcal{T}_{\text {solve }} \log (1 / \epsilon)\right)$ time a vector $x^{\prime}$ which with high probability is an approximate solution to the linear system in that $\left\|x^{\prime}-x\right\|_{\mathbf{X}} \leq \epsilon\|b\|_{\mathbf{U}^{+}}$. Furthermore, a given choice of random bits produces a correct result for all b simultaneously, and makes $x^{\prime}$ linear in $b$.

Proof. First note that we can actually choose a $\widetilde{\mathbf{U}}^{+}$satisfying the needed properties: a linearoperator based graph Laplacian solver, such as [28]. With this $\widetilde{\mathbf{U}}^{+}$, we apply Lemma 17, which produces a linear operator $\mathbf{Y}^{+}$. We simply return $x^{\prime}=\mathbf{Y}^{+} \mathcal{L}^{\top} \widetilde{\mathbf{U}}^{+} b$-corresponding to approximately solving the linear system $\mathcal{L}^{\top} \widetilde{\mathbf{U}}^{+} \mathcal{L} x=\mathcal{L}^{\top} \widetilde{\mathbf{U}}^{+} b$. The error bound follows from

$$
\begin{aligned}
\left\|x^{\prime}-x\right\|_{\mathbf{X}} & \leq\left\|x^{\prime}-x\right\|_{\widetilde{\mathbf{X}}}=\left\|\left(\mathbf{Y}^{+}-\widetilde{\mathbf{X}}^{+}\right) \mathcal{L}^{\top} \widetilde{\mathbf{U}}^{+} b\right\|_{\widetilde{X}}=\left\|\mathcal{L}^{\top} \widetilde{\mathbf{U}}^{+} b\right\|_{\left(\mathbf{Y}^{+}-\widetilde{\mathbf{X}}+\right) \widetilde{\mathbf{X}}\left(\mathbf{Y}^{+}-\widetilde{\mathbf{X}}^{+}\right)} \\
& \leq \epsilon\left\|\mathcal{L}^{\top} \widetilde{\mathbf{U}}^{+} b\right\|_{\widetilde{\mathbf{X}}^{+}}=\epsilon\left\|\widetilde{\mathbf{U}}^{+} b\right\|_{\mathcal{L}^{\mathbf{X}} \tilde{\mathcal{L}}^{+}}=\epsilon\left\|\widetilde{\mathbf{U}}^{+} b\right\|_{\widetilde{\mathbf{U}}} \\
& =\epsilon\|b\|_{\widetilde{\mathbf{U}}^{+}} \leq \epsilon\|b\|_{\mathbf{U}^{+}} .
\end{aligned}
$$

Theorem 12 follows as an immediate corollary: 
Proof of Theorem 12. Apply Lemma 18 and use the fact (from Lemma 13) that $\mathbf{U} \preceq \mathbf{X}$ :

$$
\left\|x^{\prime}-x\right\|_{\mathbf{U}} \leq\left\|x^{\prime}-x\right\|_{\mathbf{X}} \leq \epsilon\|b\|_{\mathbf{U}^{+}} .
$$

\section{Solving Strictly RCDD Systems}

Here we show how to reduce solving strictly RCDD systems using an Eulerian Laplacian solver. First we provide an prove Theorem 19 which achieves precisely this goal by using the Eulerian Laplacian system solver presented in Section 5. Then we provide Corollary 20 which specializes this result to the case of solving $\alpha$-RCDD systems. We make extensive use of this corollary to compute the the Eulerian scaling of a directed Laplacian in Section 3.

Theorem 19. Let $\mathbf{A} \in \mathcal{R}^{n \times n}$ be a strictly $R C D D Z$-matrix, let $b \in \mathcal{R}^{n}$, let $x$ be the solution to $\mathbf{A} x=b$, and let $0<\epsilon \leq \frac{1}{2}$. Then in $O\left(\mathcal{T}_{\text {solve }} \log (1 / \epsilon)\right)$ time we can compute a vector $x^{\prime}$ that satisfies $\left\|x^{\prime}-x\right\|_{\frac{1}{2}\left(\mathbf{A}+\mathbf{A}^{\top}\right)} \leq \epsilon\|b\|_{\left(\frac{1}{2}\left(\mathbf{A}+\mathbf{A}^{\top}\right)\right)^{-1}}$ with high probability.

Proof. Define the $(n+1) \times n$ matrix $\mathbf{C}=\left(\begin{array}{c}\mathbf{I}_{n} \\ -\overrightarrow{1}_{n}^{\top}\end{array}\right)$. Now define a $(n+1) \times(n+1)$ matrix $\mathcal{L}=\mathbf{C A C}^{\top}$. The top left $n \times n$ entries of $\mathcal{L}$ are a copy of $\mathbf{A}$, and its rows and columns sum to 0 since the columns of $\mathbf{C}$ sum to 0 . Since $\mathbf{A}$ is a diagonally dominant Z-matrix, the new off-diagonal entries are negative and the new diagonal entry is then positive. Thus $\mathcal{L}$ is a Z-matrix for which all rows and columns sum to to 0, so it is an Eulerian Laplacian.

Now, if $\mathbf{A} x=b$, we must have $\mathcal{L}\left(\begin{array}{l}x \\ 0\end{array}\right)=\left(\begin{array}{l}b \\ y\end{array}\right)$ for some scalar $y$, since the upper left block is A. Furthermore, this must satisfy $\overrightarrow{1}_{n}^{\top} b+y=0$, since the image of a Laplacian is orthogonal to the all-ones vector. Consequently, $\left(\begin{array}{l}b \\ y\end{array}\right)=\mathbf{C} x$. Now, for a connected Eulerian Laplacian, the null space is precisely the all-ones vector. Furthermore, $\mathcal{L}$ is clearly connected as the $n+1$ row must be non-zero by the fact that $\mathbf{A}$ is strictly RCDD. Consequently, any solution to $\mathcal{L} z=\left(\begin{array}{l}b \\ y\end{array}\right)$ must be off by only by a shift of the all-ones vector from $\left(\begin{array}{l}x \\ 0\end{array}\right)$. In particular, this implies that $x=\mathbf{C}^{\top} \mathcal{L}^{+} \mathbf{C} b$ since the $\mathbf{C}^{\top}$ adds the shift of the all-ones vector to zero out the auxiliary variable. This means that $\mathbf{A}^{-1}=\mathbf{C}^{\top} \mathcal{L}^{+} \mathbf{C}$, and by the same argument, $\left(\frac{1}{2}\left(\mathbf{A}+\mathbf{A}^{\top}\right)\right)^{-1}=\mathbf{C}^{\top}\left(\frac{1}{2}\left(\mathcal{L}+\mathcal{L}^{\top}\right)\right)^{+} \mathbf{C}=\mathbf{C}^{\top} \mathbf{U}^{+} \mathbf{C}$. This effectively reduces solving RCDD systems to solving Eulerian Laplacian systems.

Specifically, we obtain $x^{\prime}$ by calling Theorem 12 on $\mathbf{C} b$ with $\mathcal{L}$ and $\epsilon$ to get a vector $z^{\prime}$ (with $z$ referring to $\mathcal{L}^{+} \mathbf{C b}$ ), then returning $\mathbf{C}^{\top} z^{\prime}$. The desired error follows from

$$
\begin{aligned}
\left\|x^{\prime}-x\right\|_{\frac{1}{2}\left(\mathbf{A}+\mathbf{A}^{\top}\right)} & =\left\|\mathbf{C}^{\top}\left(z^{\prime}-z\right)\right\|_{\frac{1}{2}\left(\mathbf{A}+\mathbf{A}^{\top}\right)}=\left\|z^{\prime}-z\right\|_{\frac{1}{2} \mathbf{C}\left(\mathbf{A}+\mathbf{A}^{\top}\right) \mathbf{C}^{\top}}=\left\|z^{\prime}-z\right\|_{\mathbf{U}} \\
& \leq \epsilon\|\mathbf{C} b\|_{\mathbf{U}^{+}}=\epsilon\|b\|_{\mathbf{C}^{\top} \mathbf{U}^{+} \mathbf{C}}=\epsilon\|b\|_{\left(\frac{1}{2}\left(\mathbf{A}+\mathbf{A}^{\top}\right)\right)^{-1}} .
\end{aligned}
$$

Corollary 20. Let $\mathbf{A} \in \mathcal{R}^{n \times n}$ be a $\alpha$-RCDD Z-matrix, let $\mathbf{D}$ represent the matrix with $\mathbf{D}_{i j}=0$ for $i \neq j$ and $\mathbf{D}_{i i}=\frac{A_{i i}}{1+\alpha}$, let $x$ be the solution to $\mathbf{A} x=b$ for $b \in \mathcal{R}^{n}$, and let $0<\epsilon \leq \frac{1}{2}$. Then in $O\left(\mathcal{T}_{\text {solve }} \log (1 / \epsilon)\right)$ time we can compute a vector $x^{\prime}$ satisfying $\left\|x^{\prime}-x\right\|_{\mathbf{D}} \leq \frac{\epsilon}{\alpha}\|b\|_{\mathbf{D}^{-1}}$ with high probability. 
Proof. We apply Theorem 19 to our input. We can express $\frac{1}{2}\left(\mathbf{A}+\mathbf{A}^{\top}\right)$ as $\alpha \mathbf{D}+\mathbf{M}$, where $\mathbf{M}$ is a symmetric diagonally dominant, and thus positive definite, matrix. In other words we have

$$
\frac{1}{2}\left(\mathbf{A}+\mathbf{A}^{\top}\right) \succeq \alpha \mathbf{D}
$$

meaning that the $\frac{1}{2}\left(\mathbf{A}+\mathbf{A}^{\top}\right)$ norm dominates the $\alpha \mathbf{D}$ norm while the $\left(\frac{1}{2}\left(\mathbf{A}+\mathbf{A}^{\top}\right)\right)^{-1}$ norm is dominated by the $\alpha^{-1} \mathbf{D}^{-1}$ norm. Thus we have

$$
\begin{aligned}
\left\|x^{\prime}-x\right\|_{\mathbf{D}} & =\alpha^{-\frac{1}{2}}\left\|x^{\prime}-x\right\|_{\alpha \mathbf{D}} \leq \alpha^{-\frac{1}{2}}\left\|x^{\prime}-x\right\|_{\frac{1}{2}\left(\mathbf{A}+\mathbf{A}^{\top}\right)} \leq \alpha^{-\frac{1}{2}} \epsilon\|b\|_{\left(\frac{1}{2}\left(\mathbf{A}+\mathbf{A}^{\top}\right)\right)^{-1}} \\
& \leq \alpha^{-\frac{1}{2}} \epsilon\|b\|_{\alpha^{-1} \mathbf{D}^{-1}}=\frac{\epsilon}{\alpha}\|b\|_{\mathbf{D}^{-1}} .
\end{aligned}
$$

\section{Condition Number Bounds}

For the various applications of this paper (Section 7) we use three quantities to measure the degeneracy or condition number of the matrices involved. Here we define these quantities and prove that they are equivalent up to polynomial factors. Since the running times for all algorithms provided in this paper depend on these quantities only poly-logarithmically, this allows us to use whichever quantity simplifies the analysis while only mildly affecting performance.

We define these quantities for a directed Laplacian, $\mathcal{L}=\mathbf{I}-\mathbf{W} \in \mathcal{R}^{n \times n}$, where $\mathbf{W} \in \mathcal{R}_{\geq 0}^{n \times n}$ is the random walk matrix associated with a strongly connected graph. Note that for any Laplacian, $\mathcal{L}=\mathbf{D}-\mathbf{A}^{\top}$, we have $\mathcal{L} \mathbf{D}^{-1}=\mathbf{I}-\mathbf{W}$; thus it is easy to relate these quantities to those of an arbitrary Laplacian, since $\kappa(\mathbf{D}-\mathbf{A}) \leq \kappa\left(\mathbf{I}-\mathbf{A D}^{-1}\right) \cdot \kappa(\mathbf{D})$.

Inverse of Second Smallest Singular Value: This quantity is $\left\|\mathcal{L}^{+}\right\|_{2}$. Note that since $\|\mathcal{L}\|_{2} \leq$ $2 n$, by Lemma 61 we have $\kappa(\mathcal{L}) \leq 2 n\left\|\mathcal{L}^{+}\right\|_{2}$. Thus, up to a polynomial dependence on $n$, this quantity bounds the condition number of $\mathcal{L}$.

(Lazy) Mixing Time: This quantity is denoted $t_{m i x}$. It is defined as the smallest $k$ such that $\left\|\widetilde{\mathbf{W}}^{k} p-s\right\|_{1} \leq \frac{1}{2}$, for all $p \in \Delta^{n}$, where $\widetilde{\mathbf{W}} \stackrel{\text { def }}{=} \frac{1}{2}(\mathbf{I}+\mathbf{W})$ is the lazy random walk matrix associated with $\mathbf{W}$, and $s \in \Delta^{n}$ is the stationary distribution associated with $\mathbf{W}$.

Personalized PageRank Mixing Time: This quantity is denoted $t_{p p}$. It is defined as the smallest $k \geq 0$ such that, setting $\beta=\frac{1}{k}$, one has $\left\|\mathbf{M}_{p p(\beta)} p-s\right\|_{1} \leq \frac{1}{2}$, for all $p \in \Delta^{n}$; the matrix $\mathbf{M}_{p p(\beta)}=\beta(\mathbf{I}-(1-\beta) \mathbf{W})^{-1}$ is the personalized PageRank matrix with restart probability $\beta$ (See Section 2), and $s$ is the stationary distribution associated with $\mathbf{W}$. Note that $\mathbf{M}_{p p(\beta)} p$ is the personalized PageRank vector with restart probability $\beta$ and vector $p$.

Each of these quantities is required for our applications, and they are involved in the running time guarantees we provide. The inverse of the second smallest singular value is related to the condition number of $\mathcal{L}$, and is used to bound the accuracy to which we need to solve various linear systems. The mixing time is related to many applications involving directed Markov chains. In order to obtain bounds on these quantities, we use the personalized PageRank mixing time, which can be computed accurately enough for our purposes (See Section 7.2). 
The main result of this section is to prove the following theorem showing how these different quantities are related. Rather than relating many of the quantities to $\left\|\mathcal{L}^{+}\right\|_{2}$ directly, we instead obtain cleaner bounds by relating them to the maximum column norm of $\mathcal{L}$, i.e. $\left\|\mathcal{L}^{+}\right\|_{1}$. Since for $x \in \mathcal{R}^{n}$ we have $\frac{1}{\sqrt{n}}\|x\|_{1} \leq\|x\|_{2} \leq\|x\|_{1}$, it is easy to see that $\frac{1}{\sqrt{n}}\left\|\mathcal{L}^{+}\right\|_{1} \leq\left\|\mathcal{L}^{+}\right\|_{2} \leq \sqrt{n}\left\|\mathcal{L}^{+}\right\|_{1}$.

Theorem 21. Let $\mathcal{L}=\mathbf{I}-\mathbf{W} \in \mathcal{R}^{n \times n}$ be a directed Laplacian, where $\mathbf{W} \in \mathcal{R}_{\geq 0}^{n \times n}$ is a random walk matrix associated with a strongly connected graph. The inverse of the second smallest singular value of $\mathcal{L},\left\|\mathcal{L}^{+}\right\|_{2}$, the mixing time, $t_{m i x}$, the personalized PageRank mixing time, $t_{p p}$, and the largest column norm of $\mathcal{L}^{+},\left\|\mathcal{L}^{+}\right\|_{1}$, obey the following:

- Mixing Time vs. Column Norm: $\frac{1}{16} \sqrt{t_{\text {mix }}} \leq\left\|\mathcal{L}^{+}\right\|_{1} \leq t_{m i x} \cdot 4 \sqrt{n} \log _{2} n$

- Personalized PageRank Mixing vs. Singular Values: $\frac{1}{8} t_{p p} \leq\left\|\mathcal{L}^{+}\right\|_{1} \leq t_{p p} \cdot 16 \sqrt{n} \log _{2} n$

- Mixing Time vs. Personalized PageRank Mixing Time: $t_{p p} \leq 36 \cdot t_{m i x}$

- Column Norm vs. Singular Values: $\frac{1}{\sqrt{n}}\left\|\mathcal{L}^{+}\right\|_{1} \leq\left\|\mathcal{L}^{+}\right\|_{2} \leq \sqrt{n}\left\|\mathcal{L}^{+}\right\|_{1}$

Note that the analysis of our applications only rely on the relationship between $t_{p p}$ and $\left\|\mathcal{L}^{+}\right\|_{1}$ that we provide in this section. However, we provide this full set of relationships both for completeness and to admit clean and more easily understood running times.

Our proof of this theorem is split into several parts. First, in Section 6.1 we provide an operator view of mixing times, and use this to prove the relationship between personalized PageRank mixing time and mixing time. Then in Section 6.2 we prove upper bounds on $\left\|\mathcal{L}^{+}\right\|_{1}$, and in Section 6.3 we prove lower bounds on $\left\|\mathcal{L}^{+}\right\|_{1}$. Along the way we provide a more fine grained understanding of personalized PageRank mixing time (Lemma 27 and Lemma 31) that may be of further use.

\subsection{Bounds Between Mixing Times}

Here we provide bounds between different possible notions of mixing times. First we provide Lemma 22, a general mathematical lemma which implies that mixing time conditions are equivalent to certain operator norm statements on matrices, e.g. $\left\|\mathbf{W}^{k} p-s\right\|_{1} \leq \frac{1}{2}$ for all $p \in \Delta$ implies $\left\|\mathbf{W}-s \overrightarrow{1}^{\top}\right\|_{1} \leq \frac{1}{2}$. Using this we prove Lemma 23 which shows how the mixing amount of a random walk can be amplified, e.g. given $t_{\text {mix }}$ we can compute bounds on the smallest $k$ for which $\left\|\mathbf{W}^{k} p-s\right\|_{1} \leq \epsilon$, for any small enough $\epsilon$. Finally, we conclude this section with Lemma 24, which relates $t_{m i x}$ to $t_{p p}$.

Lemma 22. For all $\mathbf{A} \in \mathcal{R}^{m \times n}, b \in \mathcal{R}^{n}$, and $\alpha \geq 0$, one has that

$$
\|\mathbf{A} p-b\|_{1} \leq \alpha, \text { for all } p \in \Delta^{n},
$$

if and only if

$$
\left\|\mathbf{A}-b \overrightarrow{1}^{\top}\right\|_{1} \leq \alpha .
$$

Proof. Suppose $\left\|\mathbf{A}-b \overrightarrow{1}^{\top}\right\|_{1} \leq \alpha$. Then, for all $p \in \Delta^{n}$,

$$
\|\mathbf{A} p-b\|_{1}=\left\|\left(\mathbf{A}-b \overrightarrow{1}^{\top}\right) p\right\|_{1} \leq \alpha\|p\|_{1}=\alpha
$$

yielding one direction of the claim.

On the other hand, suppose $\|\mathbf{A} p-b\|_{1} \leq \alpha$ for all $p \in \Delta^{n}$. Let $x \in \mathcal{R}^{n}$ be arbitrary. Decompose $x$ into its positive and negative coordinates, i.e. define $x_{+}, x_{-} \in \mathcal{R}_{\geq 0}^{n}$ as the unique vectors such 
that $x=x_{+}-x_{-}$, and at most one of $x_{+}$and $x_{-}$is non-zero in a coordinate. Now, if $\left\|x_{+}\right\|_{1}=0$ or $\left\|x_{-}\right\|_{1}=0$ then for some $s \in\{-1,1\}$ we have $s \cdot x /\|x\|_{1} \in \Delta^{n}$ and

$$
\left\|\left(\mathbf{A}-b \overrightarrow{1}^{\top}\right) x\right\|_{1}=\|x\|_{1} \cdot\left\|\mathbf{A} \frac{x}{\|x\|_{1}}-b\right\|_{1} \leq\|x\|_{1} \cdot \alpha .
$$

Otherwise, by triangle inequality

$$
\begin{aligned}
\left\|\left(\mathbf{A}-b \overrightarrow{1}^{\top}\right) x\right\|_{1} & \leq\left\|\left(\mathbf{A}-b \overrightarrow{1}^{\top}\right) x_{+}\right\|_{1}+\left\|\left(\mathbf{A}-b \overrightarrow{1}^{\top}\right) x_{-}\right\|_{1} \\
& =\left\|x_{+}\right\|_{1} \cdot\left\|\mathbf{A} \frac{x_{+}}{\left\|x_{+}\right\|}-b\right\|_{1}+\left\|x_{-}\right\|_{1} \cdot\left\|\mathbf{A} \frac{x_{-}}{\left\|x_{-}\right\|}-b\right\|_{1} .
\end{aligned}
$$

Since $\frac{x_{+}}{\left\|x_{+}\right\|} \in \Delta^{n}$ and $\frac{x_{-}}{\left\|x_{-}\right\|} \in \Delta^{n}$, we have $\left\|\mathbf{A} \frac{x_{+}}{\left\|x_{+}\right\|}-b\right\|_{1} \leq \alpha$ and $\left\|\mathbf{A} \frac{x_{-}}{\left\|x_{-}\right\|}-b\right\|_{1} \leq \alpha$. Furthermore, since $\|x\|_{1}=\left\|x_{+}\right\|_{1}+\left\|x_{-}\right\|_{1}$ we have that $\left\|\left(\mathbf{A}-b \overrightarrow{1^{\top}}\right) x\right\|_{1} \leq\|x\|_{1} \cdot \alpha$. In either case, the claim holds.

Lemma 23. Let $\mathbf{W} \in \mathcal{R}_{\geq 0}^{n \times n}$ be a random walk matrix associated with a strongly connected graph. Let $s$ be its stationary distribution, and let $t_{m i x}$ be its mixing time. Then for all $k \geq t_{m i x}$ and $x \perp s$ we have

$$
\left\|\mathbf{W}^{k} x-s\right\|_{1} \leq\left(\frac{1}{2}\right)^{\left\lfloor\frac{k}{t_{m i x}}\right\rfloor} \quad \text { and } \quad\left\|\mathbf{W}^{k}-s \overrightarrow{1}^{\top}\right\|_{1} \leq\left(\frac{1}{2}\right)^{\left\lfloor\frac{k}{t_{m i x}}\right\rfloor}
$$

Proof. First note that, since $\mathbf{W} s=s$, it is the case that $\mathbf{W}^{k} x-s=\mathbf{W}^{k}(x-s)$. Furthermore, since $\|\mathbf{W}\|_{1}=1$, we have that

$$
\left\|\mathbf{W}^{k+1} x-s\right\|_{1}=\left\|\mathbf{W}\left(\mathbf{W}^{k} x-s\right)\right\|_{1} \leq\left\|\mathbf{W}^{k} x-s\right\|_{1} .
$$

Consequently, $\left\|\mathbf{W}^{k} x-s\right\|_{1}$ decreases monotonically with $k$ and by Lemma 22 it therefore suffices to prove the claim for $k=c \cdot t_{m i x}$ for positive integers.

Next, by the definition of $t_{m i x}$ and Lemma 22 we know that $\left\|\mathbf{W}-s \overrightarrow{1}^{\top}\right\|_{1} \leq \frac{1}{2}$. Furthermore, since $\overrightarrow{1}^{\top} \mathbf{W}=\overrightarrow{1}^{\top}$ and since $\mathbf{W} s=s$, for all non-negative integers $\alpha$, we have that

$$
\left(\mathbf{W}^{\alpha}-s \overrightarrow{1}^{\top}\right)(\mathbf{W}-s \overrightarrow{1})=\mathbf{W}^{\alpha+1}-s \overrightarrow{1}^{\top} \mathbf{W}-\mathbf{W}^{\alpha} s \overrightarrow{1}+s \overrightarrow{1}^{\top} s \overrightarrow{1}=\mathbf{W}^{\alpha+1}-s \overrightarrow{1}^{\top} .
$$

By induction, we know that for all non-negative integers $c$ it is the case that

$$
\left\|\mathbf{W}^{c \cdot t_{m i x}}-s \overrightarrow{1}^{\top}\right\|_{1}=\left\|\left(\mathbf{W}^{t_{m i x}}-s \overrightarrow{1}^{\top}\right)^{c}\right\|_{1} \leq\left(\frac{1}{2}\right)^{c}
$$

Applying Lemma 22 again yields the result.

We also show the following bound based on amplifying mixed walks:

Lemma 24. Let $\mathbf{W} \in \mathcal{R}_{\geq 0}^{n \times n}$ be a random walk matrix associated with a strongly connected graph, and let $s$ and be its stationary distribution, and let $t_{m i x}$ be its mixing time. Let $\mathbf{M}_{p p(\beta)}$ be the personalized PageRank matrix with restart probability $\beta$ associated with $\mathbf{W}$. For all $k \geq 1, \beta \leq$ $\left(k \cdot t_{\text {mix }}\right)^{-1}, x \in \Delta^{n}$, we have $\left\|\mathbf{M}_{p p(\beta)} x-s\right\|_{1} \leq 3 k^{-1 / 2}$. Consequently, the personalized PageRank mixing time $t_{p p}$ obeys $t_{p p} \leq 36 \cdot t_{m i x}$. 
Proof. First, a little algebraic manipulation reveals that for $\gamma=\frac{\beta}{2-\beta}$,

$$
\mathbf{I}-(1-\beta) \mathbf{W}=(2-\beta) \mathbf{I}-2(1-\beta) \widetilde{\mathbf{W}}=(2-\beta) \cdot\left(\mathbf{I}-2\left(\frac{1-\beta}{2-\beta}\right) \widetilde{\mathbf{W}}\right)=\frac{\beta}{\gamma}(\mathbf{I}-(1-\gamma) \widetilde{\mathbf{W}}) .
$$

Consequently, since $(\mathbf{I}-(1-\gamma) \widetilde{\mathbf{W}}) \frac{1}{\gamma} s=s$ we have

$$
\mathbf{M}_{p p(\beta)} x-s=\gamma(\mathbf{I}-(1-\gamma) \widetilde{\mathbf{W}})^{-1}(x-s)=\gamma \sum_{i=0}^{\infty}(1-\gamma)^{i} \widetilde{\mathbf{W}}^{i}(x-s) .
$$

Now, by triangle inequality, we know that

$$
\left\|\mathbf{M}_{p p(\beta)} x-s\right\|_{1} \leq \gamma \sum_{i=0}^{\infty}(1-\gamma)^{i}\left\|\mathbf{W}^{i}(x-s)\right\|_{1} .
$$

Since, $\left\|\mathbf{W}^{i}\right\|_{1} \leq 1$ and $\|x-s\|_{1} \leq 2$, we have that the first $\left\lfloor\sqrt{k} t_{m i x}\right\rfloor$ terms sum to at most $2 \gamma \sqrt{k} t_{\text {mix }} \leq 2 k^{-1 / 2}$. Furthermore, Lemma 23 implies that for all $i>\left\lceil\sqrt{k} t_{\text {mix }}\right\rceil$ we have

$$
\left\|\mathbf{W}^{k} x-s\right\|_{1} \leq\left(\frac{1}{2}\right)^{\left\lfloor\frac{i}{t_{m i x}}\right\rfloor} \leq 2^{-\lfloor\sqrt{k}\rfloor} \leq \frac{1}{\sqrt{k}}
$$

for $k \geq 1$, and therefore the remaining terms sum to at most $k^{-1 / 2}$.

\subsection{Singular Value Lower Bounds}

Here we show how to lower bound $\left\|\mathcal{L}^{+}\right\|_{1}$ in terms of the mixing time and personalized PageRank mixing time. First we provide a small technical lemma, Lemma 25, that will be used to reason about vectors orthogonal to stationary distribution. Then, using this result, in Lemma 26 we lower bound $\left\|\mathcal{L}^{+}\right\|_{1}$ by the mixing time, and in Lemma 27 we lower bound $\left\|\mathcal{L}^{+}\right\|_{1}$ by the personalized PageRank mixing time.

Lemma 25. For all $a, b \in \Delta^{n}$ and $\alpha \in \mathcal{R}$, we have that

$$
\|a-\alpha b\|_{1} \geq \frac{1}{2}\|a-b\|_{1} .
$$

Proof. If $|1-\alpha| \geq \frac{1}{2}\|a-b\|_{1}$ then

$$
\|a-\alpha b\|_{1} \geq\left|\sum_{i \in[n]} a_{i}-\alpha b_{i}\right|=|1-\alpha| \geq \frac{1}{2}\|a-b\|_{1} .
$$

On the other hand if $|1-\alpha| \leq \frac{1}{2}\|a-b\|_{1}$ then by reverse triangle inequality we have

$$
\|a-\alpha b\|_{1} \geq\|a-b\|_{1}-\|(\alpha-1) b\|_{1} \geq\|a-b\|_{1}-|1-\alpha| \geq \frac{1}{2}\|a-b\|_{1} .
$$

Lemma 26. For a directed Laplacian $\mathcal{L}=\mathbf{I}-\mathbf{W}$ associated with a strongly connected graph, the mixing time of the random walk matrix $\mathbf{W}$ satisfies

$$
\sqrt{t_{\text {mix }}} \leq 16\left\|\mathcal{L}^{+}\right\|_{1}
$$


Proof. Consider some vector $x \in \Delta^{n}$ that has not mixed at step $k=t_{m i x}-1$, i.e. $\left\|\mathbf{W}^{k} x-s\right\|_{1}>\frac{1}{2}$. Let

$$
y \stackrel{\text { def }}{=} \mathbf{W}^{k} x-s \cdot \frac{s^{\top} \mathbf{W}^{k} x}{\|s\|_{2}^{2}} .
$$

By construction, we have that $s^{\top} y=0$ and therefore

$$
\frac{\|y\|_{1}}{\|\mathcal{L} y\|_{1}} \leq \max _{z \perp s: z \neq 0} \frac{\|z\|_{1}}{\|\mathcal{L} z\|_{1}}=\max _{z \neq \overrightarrow{0}} \frac{\left\|\mathcal{L}^{+} z\right\|_{1}}{\|z\|_{1}}=\left\|\mathcal{L}^{+}\right\|_{1} .
$$

Now by Lemma 25 and our assumption on $x$, we know that $\|y\|_{1} \geq \frac{1}{4}$, so all that remains is to upper bound $\|\mathcal{L} y\|_{1}$. However, since $\mathcal{L} s=\overrightarrow{0}$ we have

$$
\begin{aligned}
\|\mathcal{L} y\|_{1} & =\|(\mathbf{I}-\mathbf{W}) \widetilde{\mathbf{W}} x\|_{1}=\left\|(\mathbf{I}-\mathbf{W})\left(\frac{\mathbf{I}+\mathbf{W}}{2}\right)^{k} x\right\|_{1}=\left\|\frac{\mathbf{I}-\mathbf{W}}{2^{k}} \sum_{i=0}^{k}\left(\begin{array}{l}
k \\
i
\end{array}\right) \mathbf{W}^{i} x\right\|_{1} \\
& =\frac{1}{2^{k}}\left\|\left(\mathbf{I}-\mathbf{W}^{k+1}+\sum_{i=1}^{k}\left(\left(\begin{array}{c}
k \\
i-1
\end{array}\right)-\left(\begin{array}{c}
k \\
i
\end{array}\right)\right) \mathbf{W}^{i}\right) x\right\|_{1} \\
& \leq \frac{1}{2^{k}}\left(2+\sum_{i=1}^{k}\left|\left(\begin{array}{c}
k \\
i-1
\end{array}\right)-\left(\begin{array}{c}
k \\
i
\end{array}\right)\right|\right) .
\end{aligned}
$$

In the last line we used the fact that $\|\mathbf{W}\|_{1}=1$, and thus $\left\|\mathbf{W}^{i} x\right\|_{1} \leq 1$ for all $i \geq 0$.

Now, consider the function $f(i) \stackrel{\text { def }}{=}\left(\begin{array}{c}k \\ i\end{array}\right)$. Clearly $f(i) \geq 1$, and for all $1 \leq i \leq \mathrm{k}, f(i)$ increases monotonically with $i$ until it achieves its maximum value and then decreases monotonically, and $f(i)=f(k-i)$. Consequently,

$$
\sum_{i=1}^{k}\left|\left(\begin{array}{c}
k \\
i-1
\end{array}\right)-\left(\begin{array}{c}
k \\
i
\end{array}\right)\right|=-2+2 \cdot \max _{1 \leq i \leq k} \frac{k !}{i !(k-i) !}=-2+2 \frac{k !}{\left\lfloor\frac{k}{2}\right\rfloor !\left\lceil\frac{k}{2}\right\rceil !} .
$$

Using Stirling's approximation $\sqrt{2 \pi} \cdot n^{n+\frac{1}{2}} e^{-n} \leq n ! \leq e \cdot n^{n+\frac{1}{2}} e^{-n}$ we have that if $k$ is even then

$$
\frac{k !}{\left\lfloor\frac{k}{2}\right\rfloor !\left\lceil\frac{k}{2}\right\rceil !} \leq \frac{e \cdot k^{k+\frac{1}{2}} e^{-k}}{2 \pi \cdot\left(\frac{k}{2}\right)^{k+1} e^{-k}}=\frac{e \cdot 2^{k+1}}{2 \pi \sqrt{k}} .
$$

Since this maximum binomial coefficient increases as $k$ increases, we have that this inequality holds for all $k$ if we replace $k$ in the right hand side with $k+1$, and we have

$$
\|\mathcal{L} y\|_{1} \leq \frac{1}{2^{k}}\left(2 \cdot \frac{e \cdot 2^{k+2}}{2 \pi \sqrt{k+1}}\right)=\frac{4 e}{\pi} \cdot \frac{1}{\sqrt{k+1}} \leq \frac{4}{\sqrt{k+1}} .
$$

Combining this with (6.1) and the facts that $\|y\|_{1} \geq \frac{1}{4}$ and $k=t_{\text {mix }}-1$ yields the result.

Lemma 27. Let $\mathcal{L}=\mathbf{I}-\mathbf{W}$ be a directed Laplacian associated with a strongly connected graph, and let $\mathbf{M}_{p p(\beta)}$ denote the personalized PageRank matrix with restart probability $\beta \in[0,1]$ associated with $\mathbf{W}$. For all $x \in \Delta^{n}$ we have

$$
\frac{1}{4 \beta}\left\|\mathbf{M}_{p p(\beta)} x-s\right\|_{1} \leq\left\|\mathcal{L}^{+}\right\|_{1}
$$

and consequently the personalized PageRank mixing time for $\mathbf{W}$, denoted $t_{p p}$, satisfies.

$$
\frac{t_{p p}}{8}<\left\|\mathcal{L}^{+}\right\|_{1} \quad \text { and } \quad \frac{1}{4 \beta}\left\|\mathbf{M}_{p p(\beta)}-s \overrightarrow{1}^{\top}\right\|_{1} \leq\left\|\mathcal{L}^{+}\right\|_{1} .
$$


Proof. Let $y=\mathbf{M}_{p p(\beta)} x$. Recall that this implies $y=\beta(\mathbf{I}-(1-\beta) \mathbf{W})^{-1} x=\beta \sum_{i=0}^{\infty}(1-\beta)^{i} \mathbf{W}^{i} x$, and since $x \in \Delta^{n}$ clearly $y \in \Delta^{n}$. Now let $z=y-\alpha s$ where $\alpha=s^{\top} y /\|s\|_{2}^{2}$ so that $s^{\top} z=0$.. Since $\|y-s\|_{1}>\epsilon$ by Lemma 25 , we have that $\|z\|_{1}>\frac{\epsilon}{2}$. Furthermore, since $(\mathbf{I}-(1-\beta) \mathbf{W}) y=\beta x$, we have $\mathcal{L} y=\beta(x-y)$. Also, since $\mathcal{L} s=0$ we have

$$
\|\mathcal{L} z\|_{1}=\|\mathcal{L} y\|_{1}=\beta\|x-y\|_{1} \leq \beta \cdot\left(\|x\|_{1}+\|y\|_{1}\right)=2 \beta .
$$

Consequently,

$$
\left\|\mathcal{L}^{+}\right\|_{1}=\max _{x \neq 0} \frac{\left\|\mathcal{L}^{+} x\right\|_{1}}{\|x\|_{1}}=\max _{x \perp s, x \neq 0} \frac{\|x\|_{1}}{\|\mathcal{L} x\|_{1}} \geq \frac{\|z\|_{1}}{\|\mathcal{L} z\|_{1}}>\frac{\epsilon}{4 \beta}
$$

Using the definition of $t_{p p}$ and applying Lemma 22 completes the results.

\subsection{Condition Number Upper Bounds}

Here we provide various upper bounds on $\left\|\mathcal{L}^{+}\right\|_{1}$ for a directed Laplacian $\mathcal{L}=\mathbf{I}-\mathbf{W}$ associated with a strongly connected directed graph. In particular we show how to upper bound $\left\|\mathcal{L}^{+}\right\|_{1}$ in terms of $t_{m i x}$ and $t_{p p}$. We achieve this by providing an even stronger lower bound on the mixing time of random walks. First, in Lemma 28 we show how to lower bound how well any distribution of random walk matrices of bounded length mixes. We then show how to amplify this bound in Lemma 29, providing stronger lower bounds. As a near immediate corollary of this lemma we upper bound $\left\|\mathcal{L}^{+}\right\|_{1}$ in terms of the lazy random walk mixing time (Lemma 30) and the personalized page rank mixing time (Lemma 31).

Lemma 28. Let $\mathbf{W} \in \mathcal{R}_{\geq 0}^{n \times n}$ be a random walk matrix associated with a strongly connected graph, and let $\mathbf{M}$ be a distribution of the walks of $\mathbf{W}$ of length at most $k$, i.e. $\mathbf{M}=\sum_{i=0}^{k} \alpha_{i} \mathbf{W}^{k}$ where $\alpha_{i} \geq 0, \sum_{i=0}^{k} \alpha_{i}=1$. If $k<\frac{\left\|\mathcal{L}^{+}\right\|}{2 \sqrt{n}}$, then $\left\|\mathbf{M}-s \overrightarrow{1}^{\top}\right\|_{1}>\frac{1}{2 \sqrt{n}}$.

Proof. Recall that

$$
\left\|\mathcal{L}^{+}\right\|_{1}=\max _{x \neq 0} \frac{\|\mathcal{L} x\|_{1}}{\|x\|_{1}}=\max _{x \perp s, x \neq 0} \frac{\|x\|_{1}}{\|\mathcal{L} x\|_{1}}=\max _{\|x\|_{1}=1, x \perp s}\|\mathcal{L} x\|_{1}^{-1}
$$

Consequently, we let $x$ be such that $\|x\|_{1}=1, x \perp s$, and $\|\mathcal{L} x\|_{1}^{-1}=\left\|\mathcal{L}^{+}\right\|_{1}$.

Now, by reverse triangle inequality and the definition of operator norm we have

$$
\left\|\mathbf{M}-s \overrightarrow{1}^{\top}\right\|_{1} \geq\left\|\left(\mathbf{M}-s \overrightarrow{1}^{\top}\right) x\right\|_{1} \geq\left\|x-s \overrightarrow{1}^{\top} x\right\|_{1}-\|\mathbf{M} x-x\|_{1} .
$$

However, since $\|x\|_{1}=\|s\|_{1}=1$ we know that

$$
\left\|x-s \overrightarrow{1}^{\top} x\right\|_{1} \geq\left\|x-s \overrightarrow{1}^{\top} x\right\|_{2} \geq\|x\|_{2} \geq \frac{1}{\sqrt{n}}\|x\|_{1}=\frac{1}{\sqrt{n}} .
$$

Furthermore we have that

$$
\|\mathbf{M} x-x\|_{1}=\left\|\sum_{i=0}^{k} \alpha_{i}\left(\mathbf{W}^{i} x-x\right)\right\|_{1} \leq \sum_{i=0}^{k} \alpha_{i}\left\|\mathbf{W}^{i} x-x\right\|_{1} \leq \max _{i \in[k]}\left\|\mathbf{W}^{i}-x\right\|_{1}
$$

and by triangle inequality and the fact that $\|\mathbf{W}\|_{1}=1$ we have that

$\left\|\mathbf{W}^{i} x-x\right\|_{1} \leq\|\mathbf{W} x-x\|_{1}+\left\|\mathbf{W}^{2} x-\mathbf{W} x\right\|_{1}+\cdots+\left\|\mathbf{W}^{k} x-\mathbf{W}^{k-1} x\right\|_{1} \leq k\|\mathbf{W} x-x\|_{1}=k\|\mathcal{L} x\|_{1}$. 
Using, that $k<\frac{\left\|\mathcal{L}^{+}\right\|_{1}}{2 \sqrt{n}}$ and $\|\mathcal{L} x\|_{1}=\left\|\mathcal{L}^{+}\right\|_{1}^{-1}$ we have

$$
\left\|\mathbf{M}-s \overrightarrow{1}^{\top}\right\|_{1} \geq \frac{1}{\sqrt{n}}-k\|\mathcal{L} x\|_{1} \geq \frac{1}{\sqrt{n}}-\frac{1}{2 \sqrt{n}}=\frac{1}{2 \sqrt{n}} .
$$

Lemma 29. Let $\mathbf{W} \in \mathcal{R}_{\geq 0}^{n \times n}$ be a random walk matrix associated with a strongly connected graph, and let $\mathbf{M}$ be a distribution of the walks of $\mathbf{W}$, i.e. $\mathbf{M}=\sum_{i=0}^{\infty} \alpha_{i} \mathbf{W}^{i}$ where $\alpha_{i} \geq 0$ and $\sum_{i=0}^{\infty} \alpha_{i}=1$. If $\left\|\mathbf{M}-s \overrightarrow{1}^{\top}\right\|_{1} \leq \epsilon<1$, then $\alpha_{i}>0$ for some $i \geq k$ where

$$
k=\frac{\left\|\mathcal{L}^{+}\right\|_{1}}{\log _{\epsilon}\left(\frac{1}{2 \sqrt{n}}\right) \cdot 2 \sqrt{n}} .
$$

Proof. Proceed by contradiction, and suppose that $\alpha_{i}=0$ for all $i \geq k$. Then we have that $\mathbf{M}=\sum_{i=0}^{k^{\prime}} \alpha_{i} \mathbf{W}^{i}$, where $\sum_{i=0}^{k^{\prime}} \alpha_{i}=1$ and $k^{\prime}<k$. Now clearly for all integers $a>0$, since $\mathbf{M} s=s$, we have $\left\|\mathbf{M}^{a}-s \overrightarrow{1}^{\top}\right\|_{1}=\left\|\left(\mathbf{M}-s \overrightarrow{1}^{\top}\right)^{a}\right\|_{1} \leq \epsilon^{a}$. Furthermore, picking $a=\log _{\epsilon}(1 /(2 \sqrt{n}))$ we have that $\left\|\mathbf{M}^{a}-s \overrightarrow{1}^{\top}\right\| \leq \frac{1}{2 \sqrt{n}}$. However, we also have $\mathbf{M}^{a}=\sum_{i=0}^{a k^{\prime}} \beta_{i} \mathbf{M}^{i}$, where $\beta_{i} \geq 0$ and $\sum_{i=0}^{a k^{\prime}} \beta_{i}=1$. Consequently, by Lemma 28 we have $a k^{\prime} \geq\left\|\mathcal{L}^{+}\right\|_{1} / 2 \sqrt{n}$. Since $k=\left\|\mathcal{L}^{+}\right\|_{1} /(a 2 \sqrt{n})$ we obtain $k^{\prime} \geq k$, contradicting $k^{\prime}<k$.

Lemma 30. Let $\mathcal{L}=\mathbf{I}-\mathbf{W} \in \mathcal{R}^{n \times n}$ be a directed Laplacian associated with a strongly connected graph and let $t_{\text {mix }}$ be its mixing time, then $\left\|\mathcal{L}^{+}\right\|_{1} \leq t_{\text {mix }} \cdot 4 \sqrt{n} \log _{2} n$.

Proof. By Lemma 22 we know $\left\|\widetilde{\mathbf{W}}^{t_{m i x}}-s \overrightarrow{1}^{\top}\right\|_{1} \leq \frac{1}{2}$. Since $\widetilde{\mathbf{W}}^{t_{m i x}}$ is a distribution of the walks of $\mathbf{W}$ of length at most $k$, by Lemma 29 we know that $t_{m i x} \geq k$ where

$$
k=\frac{\left\|\mathcal{L}^{+}\right\|_{1}}{\log _{1 / 2}\left(\frac{1}{2 \sqrt{n}}\right) \cdot 2 \sqrt{n}}=\frac{\left\|\mathcal{L}^{+}\right\|_{1}}{\left(1+\log _{2} \sqrt{n}\right) \cdot 2 \sqrt{n}} .
$$

Since when $n=1$ we have $\mathcal{L}=0$ and $\mathbf{W}-s \overrightarrow{1}^{\top}=0$ making the lemma trivially true and when $n \geq 2$ we have that $1+\log _{2}(\sqrt{n}) \leq 2 \log _{2} n$ the result follows.

Lemma 31. Let $\mathcal{L}=\mathbf{I}-\mathbf{W} \in \mathcal{R}^{n \times n}$ be a directed Laplacian associated with a strongly connected graph, and let $\mathbf{M}_{p p(\beta)}$ be the personalized PageRank matrix with restart probability $\beta$ associated with $\mathbf{W}$. If $\left\|\mathbf{M}_{p p(\beta)} x-s\right\|_{1} \leq \frac{1}{2}$ for all $x \in \Delta$, then $\left\|\mathcal{L}^{+}\right\|_{1} \leq \frac{16 \sqrt{n} \log _{2} n}{\beta}$; consequently, $\left\|\mathcal{L}^{+}\right\|_{1} \leq$ $t_{p p} \cdot 16 \sqrt{n} \log _{2} n$.

Proof. By Lemma 22 we know that $\left\|\mathbf{M}_{p p(\beta)} x-s \overrightarrow{1}^{\top}\right\|_{1} \leq \frac{1}{2}$. Now since $\mathbf{W}^{k}-s \overrightarrow{1}^{\top}=\left(\mathbf{W}-s \overrightarrow{1}^{\top}\right)^{k}$ as $\mathbf{W} s=s$, we have

$$
\mathbf{M}_{p p(\beta)}-s \overrightarrow{1}^{\top}=\beta(\mathbf{I}-(1-\beta) \mathbf{W})^{-1}-s \overrightarrow{1}^{\top}=\beta \sum_{i=0}^{\infty}(1-\beta)^{i}\left(\mathbf{W}-s \overrightarrow{1}^{\top}\right)^{i}
$$

and, for all $k$ and $x$, we have

$$
\left\|\left(\mathbf{M}_{p p(\beta)}-s \overrightarrow{1}^{\top}\right) x\right\|_{1} \geq\left\|\sum_{i=0}^{k} \beta(1-\beta)^{i}\left(\mathbf{W}-s \overrightarrow{1}^{\top}\right)^{i} x\right\|_{1}-\left\|\sum_{i=k+1}^{\infty} \beta(1-\beta)^{i}\left(\mathbf{W}-s \overrightarrow{1}^{\top}\right)^{i} x\right\|_{1}
$$


Since $\|\mathbf{W}\|_{1}=1$, we have

$$
\left\|\sum_{i=k+1}^{\infty} \beta(1-\beta)^{i}\left(\mathbf{W}-s \overrightarrow{1}^{\top}\right)^{i} x\right\|_{1} \leq(1-\beta)^{k+1}\left\|\left(\mathbf{M}_{p p(\beta)}-s \overrightarrow{1}^{\top}\right) x\right\|_{1}
$$

and, since $\left\|\mathbf{M}_{p p(\beta)} x-s \overrightarrow{1}^{\top}\right\|_{1} \leq \frac{1}{2}$, we have

$$
\left\|\sum_{i=0}^{k} \beta(1-\beta)^{i}\left(\mathbf{W}-s \overrightarrow{1}^{\top}\right)^{i} x\right\|_{1} \leq\left(1+(1-\beta)^{k+1}\right) \frac{1}{2}
$$

Since the geometric sum satisfies the identity, $\sum_{i=0}^{k}(1-\beta)^{i}=\frac{1-(1-\beta)^{k+1}}{\beta}$. We have that $\mathbf{M}=$ $\frac{1}{1-(1-\beta)^{k+1}} \sum_{i=0}^{k} \beta(1-\beta)^{i} \mathbf{W}^{i}$ is a distribution of walks of $\mathbf{W}$ of length at most $k$ with

$$
\left\|\mathbf{M} x-s \overrightarrow{1}^{\top}\right\|_{1} \leq \frac{1+(1-\beta)^{k+1}}{1-(1-\beta)^{k+1}} \cdot \frac{1}{2} \leq \frac{1+e^{-\beta k}}{1-e^{-\beta k}} \cdot \frac{1}{2} .
$$

If we pick $k=\frac{1}{\beta} \ln 5$ we have that $\left\|\mathbf{M}-s \overrightarrow{1}^{\top}\right\|_{1} \leq \frac{3}{4}$ and therefore, by Lemma 29:

$$
\frac{1}{\beta} \ln 5=k \geq \frac{\left\|\mathcal{L}^{+}\right\|_{1}}{\log _{\frac{3}{4}}\left(\frac{1}{2 \sqrt{n}}\right) \cdot 2 \sqrt{n}}=\frac{\log _{2}\left(\frac{4}{3}\right)\left\|\mathcal{L}^{+}\right\|_{1}}{\left(1+\log _{2}(2 \sqrt{n})\right) \cdot \sqrt{n}} .
$$

Since when $n=1$, we have $\mathcal{L}=0$ and $\mathbf{W}-s \overrightarrow{1}^{\top}=0$, making the lemma trivially true. When $n \geq 2$ we have that $1+\log _{2} \sqrt{n} \leq 2 \log _{2} n$; combining this with $\log _{2} \frac{4}{3} / \ln 5 \geq \frac{1}{4}$ completes the result.

\section{Applications}

In this section, we use the algorithms from the previous sections - in particular the stationary computation algorithm described in Theorem 2 and the solver for RCDD-linear systems from Corollary 20- to efficiently solve several of problems of interest. Most of these problems are related to computing random walk-related quantities of directed graphs. We emphasize that unlike all prior work for directed graphs, our results have only a polylogarithmic dependence on the condition number - or equivalently - the mixing time. We show how to efficiently compute

- $\mathbf{A}^{+} b$ where $\mathbf{A}$ is any row- or column-diagonally dominant matrix and $b$ is orthogonal to the null space of $\mathbf{A}$, without any requirement that $\mathbf{A}$ being strictly row-column-diagonally-dominant or a $\mathbf{Z}$-matrix (7.3)

- $\mathcal{L}^{+} b$ where $\mathcal{L}$ is a directed Laplacian matrix and $b$ is any vector in $\mathcal{R}^{n}$, including those not in the image of $\mathcal{L}(7.3)$

- personalized PageRank (7.1)

- the mixing time of a directed graph - up to various polynomial factors - and its stationary distribution (7.2)

- hitting times for any particular pair of vertices (7.4)

- escape probabilities for any particular triple of vertices (7.5) 
- commute times between all pairs of vertices using Johnson-Lindenstrauss sketching (7.4)

As with Theorem 2, all of our routines will utilize a solver for a RCDD linear system in a black-box manner. Therefore, the running time bounds will also involve black-box bounds on the costs of such a solver.

\subsection{Computing Personalized PageRank Vectors}

Here we show how to apply our RCDD solver to compute personalized PageRank vectors. The algorithm we provide and analyze in this section serves as the basis for many of the applications we provide throughout Section 7.

Recall that given a restart probability $\beta>0$ and a vector of teleportation probabilities $p$, the personalized PageRank vector of a Markov chain is defined as the vector $x$ satisfying

$$
\beta \cdot p+(1-\beta) \mathbf{W} x=x .
$$

Rearranging terms we have that this condition is equivalent to finding the $x$ such that

$$
\mathbf{M}_{\beta} x=p_{\beta} \quad \text { where } \quad \mathbf{M}_{\beta}=\frac{\beta}{1-\beta} \mathbf{I}+(\mathbf{I}-\mathbf{W}) \quad \text { and } \quad p_{\beta}=\frac{\beta}{1-\beta} \cdot p .
$$

Consequently it suffices to solve this linear system $\mathbf{M}_{\beta} x=p_{\beta}$.

We solve this system in three steps. First we use Theorem 2 with Corollary 20 to compute an approximate Eulerian scaling of $\mathbf{I}-\mathbf{W}$, which we denote $s$. Under this scaling we show that that $\mathbf{M}_{\beta} \mathbf{S}$ is $\alpha$-RCDD for a sufficiently small $\alpha$. Since $\mathbf{M}_{\beta} \mathbf{S}$ is $\alpha$-RCDD we can solve linear systems in it, i.e. compute a $y \approx y_{*}$ where $\mathbf{M}_{\beta} \mathbf{S} y_{*}=p_{\beta}$, by Corollary 20. Final, we output $x=\mathbf{S} y$ as our approximate personalized PageRank vector. This algorithm is stated formally in Algorithm 2 and in Lemma 32 we bound the running time of this algorithm and the quality of $x$ as an approximation personal PageRank vector.

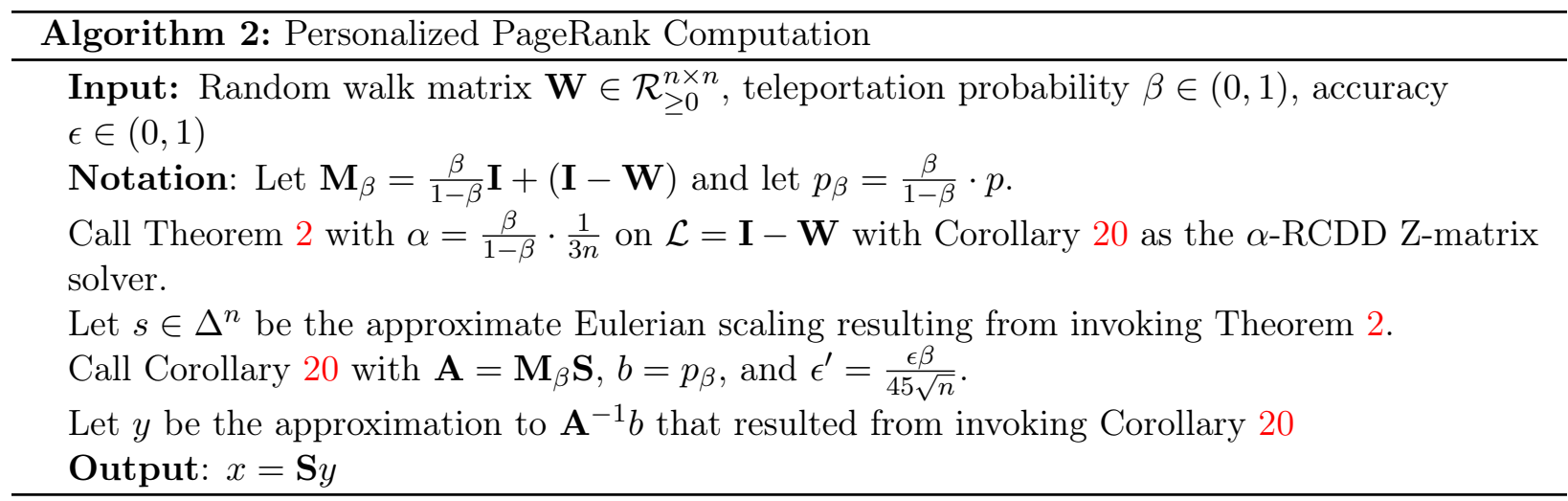

Lemma 32. (Personalized PageRank Computation) Let $\mathbf{W} \in \mathcal{R}_{\geq 0}^{n \times n}$ be a random walk matrix with $m$ non-zero entries, let $\beta \in(0,1)$ be a restart probability, let $p \in \mathcal{R}_{\geq 0}^{n}$ be teleportation constants, and let $x_{*}$ be the corresponding personalized PageRank vector. Then for any $\epsilon \in(0,1)$, Algorithm 2 computes a vector $x$ such that $\left\|x-x_{*}\right\|_{2} \leq \epsilon\|p\|_{2}$ with high probability in $n$ in time $O\left(\mathcal{T}_{\text {solve }} \cdot\left(\log ^{2} \frac{n}{\beta}+\log \frac{1}{\epsilon}\right)\right)$.

Proof. Here we analyze Algorithm 2 step by step. First, consider the invocation Theorem 2 with $\alpha=\frac{\beta}{1-\beta} \cdot \frac{1}{3 n}$ on matrix $\mathbf{I}-\mathbf{W}$. By Theorem 2 we know that the approximate Eulerian scaling $s \in \Delta^{n}$ is such that $(3 \alpha n \cdot \mathbf{I}+(\mathbf{I}-\mathbf{W})) \mathbf{S}$ is $\alpha$-RCDD and $\kappa(\mathbf{S}) \leq \frac{20}{\alpha^{2}} n$. Furthermore, since we invoke Theorem 2 using Corollary 20 as the $\alpha$-RCDD Z-matrix solve we know 
that $\mathcal{T}$ in Theorem 2 is $O\left(\mathcal{T}_{\text {solve }} \log \left(\frac{n}{\alpha}\right)\right)$. Since $\mathcal{T}_{\text {solve }}=\Omega(m)$ invoking Theorem 2 takes time $O\left(\left(m+\mathcal{T}_{\text {solve }} \log \left(\frac{n}{\alpha}\right)\right) \log \alpha^{-1}\right)=O\left(\mathcal{T}_{\text {solve }} \log ^{2}\left(\frac{n}{\beta}\right)\right)$

Next, consider the invocation of Corollary 20 on an A and $b$. Note that $\alpha$ was chosen precisely so that $(3 \alpha n \cdot \mathbf{I}+(\mathbf{I}-\mathbf{W})) \mathbf{S}=\mathbf{M}_{\beta} \mathbf{S}$ and therefore by the reasoning in the preceding paragraph we know that $\mathbf{M}_{\beta} \mathbf{S}$ is $\alpha$-RCDD and therefore we can invoke Corollary 20. By Corollary 20 we know that $\left\|y-y_{*}\right\|_{\mathbf{D}} \leq \frac{\epsilon^{\prime}}{\alpha}\left\|p_{\beta}\right\|_{\mathbf{D}^{-1}}$ where $y_{*}=\mathbf{A}^{-1} b=\mathbf{S}^{-1} \mathbf{M}_{\beta}^{-1} p_{\beta}$ and $\mathbf{D}=\left(\frac{\beta}{1-\beta} \mathbf{I}+\mathbf{I}\right) \mathbf{S}=\frac{1}{1-\beta} \mathbf{S}$. Moreover, we compute this approximation in time $O\left(\mathcal{T}_{\text {solve }} \log \left(1 / \epsilon^{\prime}\right)\right)=O\left(\mathcal{T}_{\text {solve }} \log \left(\frac{n}{\epsilon \beta}\right)\right)$ giving the overall running time.

Finally, consider the vector $x=\mathbf{S} y$ returned by the algorithm. Since $x_{*}=\mathbf{S} y_{*}$ we have

$$
\begin{aligned}
\left\|x-x_{*}\right\|_{2} & =\left\|\mathbf{S} y-\mathbf{S} y_{*}\right\|_{2} \leq \sqrt{\max _{i \in[n]} \mathbf{S}_{i i}} \cdot\left\|y-y_{*}\right\|_{\mathbf{S}}=\sqrt{(1-\beta) \cdot \max _{i \in[n]} \mathbf{S}_{i i}} \cdot\left\|y-y_{*}\right\|_{\mathbf{D}} \\
& =\frac{\epsilon^{\prime}}{\alpha} \sqrt{(1-\beta) \cdot \max _{i \in[n]} \mathbf{S}_{i i}} \cdot\left\|p_{\beta}\right\|_{\mathbf{D}^{-1}} \leq \frac{\epsilon^{\prime}}{\alpha}(1-\beta) \sqrt{\kappa(\mathbf{S})}\left\|p_{\beta}\right\|_{2}=\frac{\epsilon^{\prime}}{\alpha} \beta \sqrt{\kappa(\mathbf{S})}\|p\|_{2} .
\end{aligned}
$$

Now, using that $\alpha \geq \frac{\beta}{3 n}, \kappa(\mathbf{S}) \leq \frac{20}{\alpha^{2}} n$, and our choice of $\epsilon^{\prime}$ the result follows.

With this routine, we can also compute a good approximation of the stationary distribution via a sufficiently large $\beta$.

\subsection{Condition Number and Stationary Distribution Estimation}

Here we show how to use the PageRank computation algorithm from Section 7.1 to estimate various "condition number" like quantities associated with a directed Laplacian or a random walk matrix. We use these quantities to parameterize the running times of the algorithms presented throughout the remainder of Section 7. In particular, we use the relationships between the smallest singular value of $\mathcal{L}$, the mixing time, and the personalized PageRank mixing time proved in Section 6 to estimate each up to polynomial factors in the quantities and $n$. Furthermore, we use the bounds of Section 6 to obtain precise estimates to the stationary distribution. The key results of this section are summarized in the following theorem, whose proof we defer to the end of this Section 7.2.

Theorem 33. Let $\mathcal{L}=\mathbf{I}-\mathbf{W} \in \mathcal{R}^{n \times n}$ be directed Laplacian associated with a strongly connected graph. Let $\kappa$ be any of the mixing time of $\mathbf{W}, t_{\text {mix }}$, the personalized PageRank mixing time of $\mathbf{W}$, and $\kappa(\mathcal{L})$. Then in time $O\left(\mathcal{T}_{\text {solve }} \cdot \log ^{2}(n \cdot \kappa)\right)$ with high probability in $n$ we can compute $\tilde{\kappa}$ such that

$$
t_{p p} \leq \tilde{\kappa} \leq 400 n^{2}\left\|\mathcal{L}^{+}\right\|_{1} \text { and } n^{-1 / 2} \log _{2}^{-1 / 2}(n)\left\|\mathcal{L}^{+}\right\|_{1} \leq \tilde{\kappa} \leq 400 n^{2}\left\|\mathcal{L}^{+}\right\|_{1},
$$

and

$$
t_{p p} \leq \tilde{\kappa} \leq 6400 n^{2.5} \log _{2}(n) \cdot t_{p p} \text { and } \frac{1}{16} n^{-1 / 2} \log _{2}^{-1 / 2}(n) \sqrt{t_{m i x}} \leq \tilde{\kappa} \leq 1600 n^{2.5} \log _{2}(n) t_{m i x} .
$$

Furthermore, for any $\epsilon \in(0,1)$ in time $O\left(\mathcal{T}_{\text {solve }} \cdot \log ^{2}\left(n \cdot \epsilon^{-1}\right)\right.$ we can compute $s^{\prime}$ that approximates the stationary distribution $s$ of $\mathbf{W}$, in the sense that $\left\|s^{\prime}-s\right\|_{2} \leq \epsilon \cdot\left\|\mathcal{L}^{+}\right\|_{1}$. Consequently, if instead we spend time $O\left(\mathcal{T}_{\text {solve }} \cdot \log ^{2}\left(n \cdot \kappa \cdot \epsilon^{-1}\right)\right.$ we can obtain $\left\|s^{\prime}-s\right\|_{2} \leq \epsilon$ and if we spend time $O\left(\mathcal{T}_{\text {solve }} \cdot \log ^{2}\left(n \cdot \kappa \cdot \kappa(\mathbf{S}) \cdot \epsilon^{-1}\right)\right)$ we can obtain $(1-\epsilon) \mathbf{S} \preceq \mathbf{S}^{\prime} \preceq(1+\epsilon) \mathbf{S}$.

Note that we believe that significantly better estimates of the condition number quantities are possible. However, as our algorithms depend logarithmically on these quantities, the estimates that this theorem provides are more than sufficient to achieve our running time bounds. 
Our primary tools for proving Theorem 33 are the PageRank computation algorithm from Section 7.1. Lemma 32, and the structural results of Section 6 allow us to relate the personalized PageRank mixing time to the quantities we wish to compute. Recall from Section 6 that for a random walk matrix $\mathbf{W} \in \mathcal{R}^{n \times n}$ associated with a strongly connected graph, the personalized PageRank mixing time, $t_{p p}$ is the smallest $k$ such that for all probability vectors $x \in \Delta^{n}$,

$$
\left\|\mathbf{M}_{p p(1 / k)} x-s\right\|_{1} \leq \frac{1}{2} \quad \text { where } \quad \mathbf{M}_{p p(1 / k)} \stackrel{\text { def }}{=}(1 / k)(\mathbf{I}-(1-(1 / k)) \mathbf{W})^{-1} .
$$

Our approach is to roughly estimate this quantity and use it to compute approximations to the other condition number quantities. Unfortunately computing this quantity directly requires knowing $s$. We circumvent this using the linearity of the PageRank matrix, specifically we use that

$$
\operatorname{dist}(\beta) \stackrel{\text { def }}{=} \max _{x \in \mathcal{R}^{n}: x \perp \overrightarrow{1},\|x\|_{1} \leq 1}\left\|\mathbf{M}_{p p(\beta)} x\right\|_{1}
$$

is a constant factor approximation as shown in the following lemma.

Lemma 34. For any $\beta \in(0,1)$,

$$
\operatorname{dist}(\beta) \leq \max _{x \in \Delta^{n}}\left\|\mathbf{M}_{p p(\beta)} x-s\right\|_{1} \leq 2 \cdot \operatorname{dist}(\beta) .
$$

Proof. Note that by Lemma 22 we have that

$$
\max _{x \in \Delta}\left\|\mathbf{M}_{p p(\beta)} x-s\right\|_{1}=\left\|\mathbf{M}_{p p(\beta)} x-s \overrightarrow{1}^{\top} x\right\|_{1}=\max _{x \in \mathcal{R}^{n}:\|x\|_{1} \leq 1}\left\|\mathbf{M}_{p p(\beta)} x-s \overrightarrow{1}^{\top} x\right\|_{1} .
$$

This maximization can then be restricted to the set of vectors $x \perp 1$, giving (again by by Lemma 22):

$$
\leq \max _{x \in \mathcal{R}^{n}: x \perp 1\|x\|_{1} \leq 1}\left\|\mathbf{M}_{p p(\beta)} x-s \overrightarrow{1}^{\top} x\right\|_{1}=\max _{x \in \mathcal{R}^{n}: x \perp 1\|x\|_{1} \leq 1}\left\|\mathbf{M}_{p p(\beta)} x\right\|_{1}=\operatorname{dist}(\beta) .
$$

To show the other direction, let $x \in \Delta^{n}$ be a maximizer of $\left\|\mathbf{M}_{p p(\beta)} x-s\right\|_{1}$. Let $y=\frac{1}{2}(x-s)$. We know that

$$
\mathbf{M}_{p p(\beta)} s=\beta(\mathbf{I}-(1-\beta) \mathbf{W})^{-1} s=\beta \sum_{i=0}^{\infty}(1-\beta)^{i} \mathbf{W}^{i} s=s
$$

and therefore $\left\|\mathbf{M}_{p p(\beta)} y\right\|_{1}=\left\|\mathbf{M}_{p p(\beta)} x-s\right\|_{1}$. Furthermore, $\overrightarrow{1}^{\top} y=0$ and by triangle inequality $\|y\|_{1} \leq 1$. Consequently, $y$ can be used in the maximization problem for $\operatorname{dist}(\beta)$ and we have $\operatorname{dist}(\beta) \geq \frac{1}{2}\left\|\mathbf{M}_{p p(\beta)} x-s\right\|_{1}$.

Therefore the key to estimating $t_{p p}$ becomes estimating the $\ell_{1}$ operator norm of $\mathbf{M}_{p p(1 / k)}$ in the space orthogonal to $\overrightarrow{1}$. In the following lemma we show how to do this through random projections.

Lemma 35. Given a matrix $\mathbf{M} \in \mathcal{R}^{n \times n}$, we can determine w.h.p. in $n$ whether

$$
\max _{x \in \mathcal{R}^{n}: x \perp 1\|x\|_{1} \leq 1}\|\mathbf{M} x\|_{1} \geq \frac{1}{2} \quad \text { or } \max _{x \in \mathcal{R}^{n}: x \perp 1\|x\|_{1} \leq 1}\|\mathbf{M} x\|_{1}<\frac{1}{2 n^{2}}
$$

by:

1. Evaluating $\|\mathbf{M} x\|_{2}$ to additive error $\frac{1}{4 n}$ for $O(\log n)$ random unit vectors $x$ projected to be orthogonal to the all 1 -s vector, and 
2. Return with the first case ( $M$ having large $1 \rightarrow 1$ norm) if the result is at least $\frac{1}{4 n}$ for any of the vectors and the second otherwise.

Proof. The fact that $\|y\|_{2} \leq\|y\|_{1} \leq n^{1 / 2}\|y\|_{2}$ means we have:

$$
\max _{x \in \mathcal{R}^{n}: x \perp 1\|x\|_{1} \leq 1}\|\mathbf{M} x\|_{1} \leq n^{1 / 2} \max _{x \in \mathcal{R}^{n}: x \perp 1\|x\|_{1} \leq 1}\|\mathbf{M} x\|_{2} \leq n^{1 / 2} \max _{x \in \mathcal{R}^{n}: x \perp 1\|x\|_{2} \leq 1}\|\mathbf{M} x\|_{2},
$$

and

$$
\max _{x \in \mathcal{R}^{n}: x \perp 1\|x\|_{1} \leq 1}\|\mathbf{M} x\|_{1} \geq \max _{x \in \mathcal{R}^{n}: x \perp 1\|x\|_{1} \leq 1}\|\mathbf{M} x\|_{2} \geq n^{-1 / 2} \max _{x \in \mathcal{R}^{n}: x \perp 1\|x\|_{1} \leq 1}\|\mathbf{M} x\|_{2}
$$

Consequently, approximating the $\ell_{2}$-matrix norm gives an $n$-approximation to the desired quantity.

Furthermore, we have that for a random unit vector $x$ orthogonal to the all-1s vector, with constant probability its mass in the top eigenspace is at least $1 / \operatorname{rank}(\mathbf{M}) \geq 1 / n$ (see e.g. the proof of Theorem 7.2. of [52]) and $\|\mathbf{M} x\|_{2}$ is at least $1 / \sqrt{n}$ of the maximum. So taking $O(\log n)$ such vectors $x$ randomly and evaluating $\|\mathbf{M} x\|_{2}$ to additive error $1 / 4 n$, and checking if $\|\mathbf{M} x\|_{2} \geq \frac{1}{4 n}$ for any yields the result.

Combining this estimation routine with a binary-search-like routine leads to an estimation routine whose pseudocode is shown in Algorithm 3.

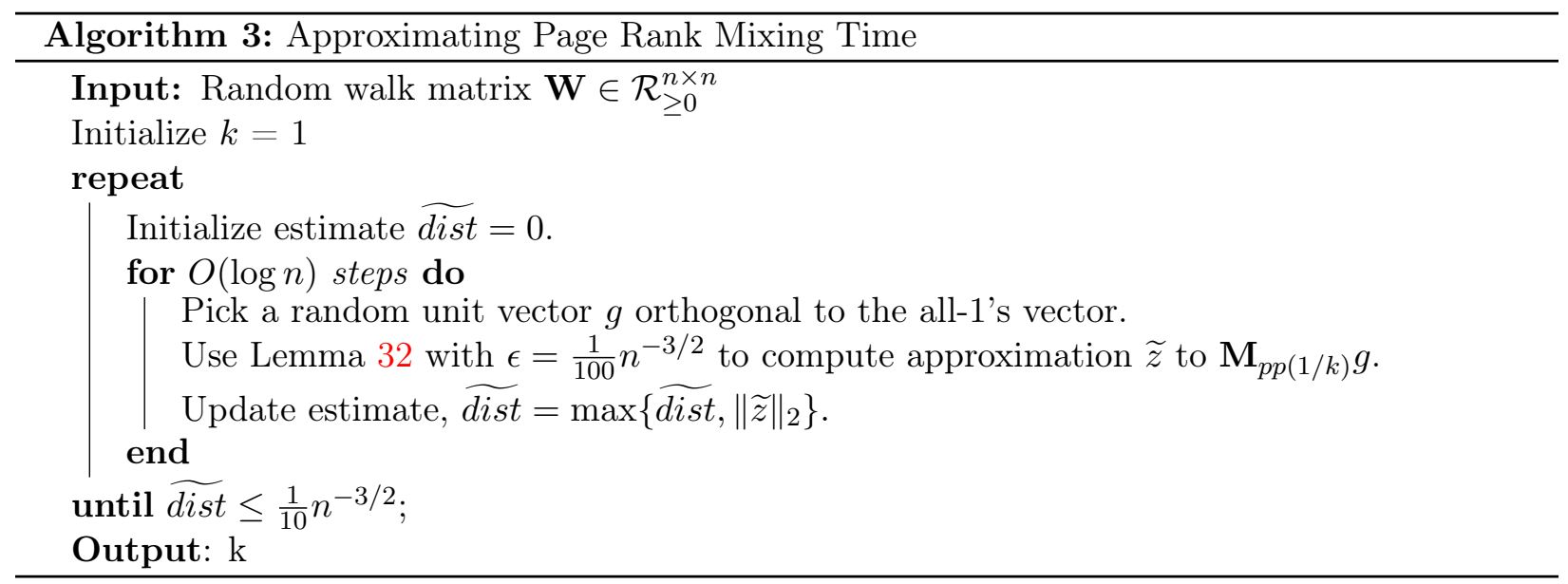

Note that since mixing is not monotonic in $k$, this is not binary search in the strictest sense. However, the relatively large multiplicative error that we allow means we can still invoke Theorem 24 to provide guarantees for this process.

Lemma 36. In $O\left(\mathcal{T}_{\text {solve }} \cdot \log ^{2}\left(n \cdot\left\|\mathcal{L}^{+}\right\|_{1}\right)\right)$ time Algorithm 3 computes a quantity $\tilde{\kappa}$ such that

$$
t_{p p} \leq \tilde{\kappa} \leq 400 n^{2}\left\|\mathcal{L}^{+}\right\|_{1}
$$

Proof. Since $\|g\|_{2} \leq 1$, the errors from the approximate personalized PageRank calls to Lemma 32 affect the results by at most an additive $\frac{1}{100} n^{-3 / 2}$. Lemma 35 gives that if $\widetilde{d i s t} \leq \frac{1}{10} n^{-3 / 2}$, we have with high probability

$$
\max _{x \in \mathcal{R}^{n}: x \perp \overrightarrow{1},\|x\|_{1} \leq 1}\left\|\mathbf{M}_{p p(1 / k)} x\right\|_{1} \leq \frac{1}{4},
$$

which by Lemma 34 implies $\max _{x \in \Delta^{n}}\left\|\mathbf{M}_{p p(\beta)} x-s\right\|_{1} \leq 1 / 2$ with high probability, and therefore $k \geq t_{p p}$. 
On the other hand, Lemma 27 gives that for any $k \geq 400 n^{2}\left\|\mathcal{L}^{+}\right\|_{1}$, we have for all $x \in \Delta^{n}$,

$$
\left\|\mathbf{M}_{p p(1 / k)} x-s\right\|_{1} \leq \frac{4}{k}\left\|\mathcal{L}^{+}\right\|_{1} \leq \frac{1}{100} n^{-2} .
$$

which by Lemma 34 implies dist $\leq \frac{1}{50} n^{-2} .35$ then gives that we will have $\widetilde{\text { dist }} \leq \frac{1}{10} n^{-2}$ with probability. This gives the desired approximation ratio of the output $\tilde{k}$. Also, the fact that we only run the approximate Personalized PageRank algorithm from Lemma 32 with $k \leq O\left(n^{2}\left\|\mathcal{L}^{+}\right\|_{1}\right)$ gives the running time bound.

We now have everything we need to prove Theorem 33. Our bounds on the other condition number quantities come from Theorem 21 and by applying Lemma 32 and Lemma 27 we obtain our stationary distribution estimation bounds. More precisely we compute a personalized PageRank vector on the uniform distribution with the restart probability small enough so that we can use Lemma 27 to reason about the error in this computation.

Proof of Theorem 33. Computing the condition number estimate, $\tilde{\kappa}$, using Lemma 36 and then applying Theorem 21 yields our bounds on $\tilde{\kappa}$. The form of the running time follows again from the equivalence of condition numbers from Lemma 36 and the fact that $\left\|\mathcal{L}^{+}\right\|_{1}=O(\operatorname{poly}(n, \kappa(\mathcal{L}))$. (See Section 6). All that remains is to compute the estimates $s^{\prime}$ to the stationary distribution. To prove the results on stationary computation, we simply compute $y \approx \mathbf{M}_{p p(\epsilon)}\left(\frac{1}{n} \overrightarrow{1}\right)$ to $\epsilon$ accuracy using Lemma 32. Since $\left\|\frac{1}{n} \overrightarrow{1}\right\|_{2}=1 / \sqrt{n}$, Lemma 32 yields that $\left\|y-\mathbf{M}_{p p(\epsilon)}\left(\frac{1}{n} \overrightarrow{1}\right)\right\|_{2} \leq \epsilon / \sqrt{n}$ and computes this in time $O\left(\mathcal{T}_{\text {solve }} \cdot \log ^{2}\left(\frac{n}{\epsilon}\right)\right.$. However, by Lemma 27 we have $\left\|\mathbf{M}_{p p(\epsilon)}\left(\frac{1}{n} \overrightarrow{1}\right)-s\right\|_{1} \leq 4 \epsilon\left\|\mathcal{L}^{+}\right\|_{1}$ and consequently

$$
\|y-s\|_{2} \leq\left\|y-\mathbf{M}_{p p(\epsilon)}\left(\frac{1}{n} \overrightarrow{1}\right)\right\|_{2}+\left\|\mathbf{M}_{p p(\epsilon)}\left(\frac{1}{n} \overrightarrow{1}\right)-s\right\|_{2} \leq \epsilon\left(1+4\left\|\mathcal{L}^{+}\right\|_{1}\right) .
$$

Since $\left\|\mathcal{L}^{+}\right\|_{1}$ is larger than some absolute constant we see that by scaling down $\epsilon$ by a constant we obtain our first result on computing an approximation to $s$. The second follows from estimating $\left\|\mathcal{L}^{+}\right\|_{1}$ from the first part of the theorem and scaling down $\epsilon$ by that. The third follows from the fact that if we have $\|y-s\|_{\infty} \leq \epsilon \cdot \min _{i \in[n]} s_{i}$ then $(1-\epsilon) \mathbf{S} \preceq \mathbf{Y} \preceq(1+\epsilon) \mathbf{S}$ and noting that we can make sure this hold by seeing if $\|y-s\|_{2} \leq \frac{\epsilon}{2} \cdot \min _{i \in[y]} y_{i}$ which must happen when $\|y-s\|_{2} \leq \frac{\epsilon}{8} \cdot \min _{i \in[n]} s_{i}$. The form of the running time follows from the fact that $\max _{i \in[n]} s_{i} \geq \frac{1}{n}$ and thus $1 / \min _{i \in[n]} s_{i i} \leq n \cdot \kappa(\mathbf{S})$.

\subsection{Solving More General Linear Systems}

Corollary 20 allows one to solve linear systems involving RCDD matrices, but requires the matrix to be strictly RCDD and have non-positive off-diagonals. Furthermore, it may return solutions with components in the null space of the matrix, while several of our subsequent applications rely on matrix-vector multiplications involving the pseudoinverse, specifically, computing vectors of the form $\mathcal{L}^{+} b$.

In this section we give removes these restrictions, at the cost of introducing a dependence on the condition number of the matrix. These proofs can be viewed as more involved analogs of the double-cover reduction from solving SDDM matrices to M matrices, and projecting away against the null space. The main complications are the much more complicated error propagation behaviors in directed settings. The following two Lemmas are crucial for handling these errors.

Lemma 37. Let $u, v$ be any vectors and $\alpha, \beta$ any scalars. Then $\|\alpha u-\beta v\|_{2} \leq|\alpha-\beta| \cdot\|u\|_{2}+\| u-$ $v \|_{2} \cdot|\beta|$. 
Proof. We have $\|\alpha u-\beta v\|_{2}=\|(\alpha-\beta) u+(u-v) \beta\|_{2} \leq|\alpha-\beta| \cdot\|u\|_{2}+\|u-v\|_{2} \cdot|\beta|$

Lemma 38. Let $\mathbf{M}=\alpha \mathbf{B}+\mathbf{A}$ be an invertible matrix with $\mathbf{M} y=b$ and $x=\mathbf{A}^{+} b$. Suppose $b \in \operatorname{im}(\mathbf{A})$. Then, $\|y-x\|_{2} \leq \alpha \cdot\left\|\mathbf{M}^{-1}\right\|_{2} \cdot\|\mathbf{B}\|_{2} \cdot\|x\|_{2}$. In particular, this implies $\|y\|_{2} \leq$ $\left(1+\alpha \cdot\left\|\mathbf{M}^{-1}\right\|_{2} \cdot\|\mathbf{B}\|_{2}\right) \cdot\|x\|_{2} \leq\left(1+\alpha \cdot\left\|\mathbf{M}^{-1}\right\|_{2} \cdot\|\mathbf{B}\|_{2}\right) \cdot\left\|\mathbf{A}^{+}\right\|_{2} \cdot\|b\|_{2}$.

Proof. Expanding yields that

$$
\|y-x\|_{2}=\left\|\mathbf{M}^{-1} b-\mathbf{A}^{+} b\right\|_{2}=\left\|\mathbf{M}^{-1}\left(\mathbf{I}-\mathbf{M} \mathbf{A}^{+}\right) b\right\|_{2}=\alpha\left\|\mathbf{M}^{-1} \mathbf{B} \mathbf{A}^{+} b\right\|_{2}=\alpha\left\|\mathbf{M}^{-1} \mathbf{B} x\right\|_{2}
$$

where in the second-to-last step, we used that $\mathbf{M}=\alpha \mathbf{B}+\mathbf{A}$ and $\mathbf{A A}^{+} b=b$ for $b \in \operatorname{im}(\mathbf{A})$.

Theorem 39. (Arbitrary RCDD system solver) Let $\mathbf{A}$ be an invertible $n \times n$ RCDD matrix. Let $b$ be an $n$-dimensional vector, and $x$ the solution to $\mathbf{A} x=b$. Let $r, u$ be the smallest and largest entries, respectively, on the diagonal of $\mathbf{A}$ so $\kappa(\mathbf{D})=u / r$. Then for any $\epsilon>0$, one can compute, with high probability and in $O\left(\mathcal{T}_{\text {solve }} \log \left(\frac{\kappa(\mathbf{A}) \cdot \kappa(\mathbf{D})}{\epsilon}\right)\right)$ time, a vector $x^{\prime}$ satisfying $\left\|x^{\prime}-x\right\|_{2} \leq \epsilon\|x\|_{2}$.

Proof. We first handle the case where the off-diagonal entries can be positive. For an $\alpha$-RCDD matrix $\mathbf{M}$, let $\mathbf{M}_{+}$denote the matrix of positive off-diagonal entries of $\mathbf{M}$, with zeros elsewhere. Let $\mathbf{M}_{-}$be the matrix of negative off-diagonals. Consider the matrix

$$
\mathbf{Z}=\left[\begin{array}{cc}
\operatorname{diag}(\mathbf{M})+\mathbf{M}_{-} & -\mathbf{M}_{+} \\
-\mathbf{M}_{+} & \operatorname{diag}(\mathbf{M})+\mathbf{M}_{-}
\end{array}\right]
$$

and the system

$$
\mathbf{Z}\left[\begin{array}{l}
x_{1} \\
x_{2}
\end{array}\right]=\left[\begin{array}{c}
b \\
-b
\end{array}\right]
$$

Note that $\mathbf{Z}$ is RCDD and a Z-matrix. Let $y$ be the solution to $\mathbf{M} y=b$. Then the solution $x_{1}=-x_{2}=x$ satisfies this new system. Similarly, let $\left(x_{1} ; x_{2}\right)$ be a solution to this new system, where the semicolon denotes vertical concatenation. Then $x=\left(x_{1}-x_{2}\right) / 2$ is a solution to the original system. Now, suppose we have an approximate solution $\left(x_{1}^{\prime} ; x_{2}^{\prime}\right)$ to the new system in the sense of Corollary 20. Define $\left(e_{1} ; e_{2}\right)=\left(x_{1}^{\prime}-x_{1} ; x_{2}^{\prime}-x_{2}\right)$. Let $\mathbf{D}=\operatorname{diag}(\mathbf{M}) /(1+\alpha)$. Note that

$$
2 \operatorname{diag}(\mathbf{Z})-\left[\begin{array}{c}
\mathbf{I} \\
-\mathbf{I}
\end{array}\right] \mathbf{D}\left[\begin{array}{ll}
\mathbf{I} & -\mathbf{I}
\end{array}\right]=\left[\begin{array}{l}
\mathbf{I} \\
\mathbf{I}
\end{array}\right] \mathbf{D}\left[\begin{array}{ll}
\mathbf{I} & \mathbf{I}
\end{array}\right] \succeq \mathbf{0}
$$

and consequently, the approximate solution $x^{\prime}=\frac{x_{1}^{\prime}-x_{2}^{\prime}}{2}$ to $\mathbf{A} x=b$ satisfies

$$
\begin{aligned}
\left\|x^{\prime}-x\right\|_{\mathbf{D}} & =\frac{1}{2} \cdot\left[\begin{array}{ll}
e_{1}^{\top} & e_{2}^{\top}
\end{array}\right]\left[\begin{array}{c}
\mathbf{I} \\
-\mathbf{I}
\end{array}\right] \mathbf{D}\left[\begin{array}{ll}
\mathbf{I} & -\mathbf{I}
\end{array}\right]\left[\begin{array}{c}
e_{1} \\
e_{2}
\end{array}\right] \leq\left[\begin{array}{ll}
e_{1}^{\top} & e_{2}^{\top}
\end{array}\right] \operatorname{diag}(\mathbf{Z})\left[\begin{array}{l}
e_{1} \\
e_{2}
\end{array}\right] \\
& =\left\|\left(e_{1} ; e_{2}\right)\right\|_{\operatorname{diag}(\mathbf{Z})} \leq\|(b ;-b)\|_{\operatorname{diag}(\mathbf{Z})^{-1}}=\|b\|_{\mathbf{D}^{-1},}
\end{aligned}
$$

which is, up to a constant factor, exactly the error guarantee of Corollary 20.

With the reduction now complete, let $\mathbf{D}=\operatorname{diag}(\mathbf{A})$. Set $\mathbf{M}=\alpha \mathbf{D}+\mathbf{A}$ for $\alpha=\frac{\epsilon r}{4 \cdot\left\|\mathbf{A}^{-1}\right\|_{2}^{2} \cdot u^{2}}$. Note that $\mathbf{M}$ is $\alpha$-RCDD. Since $\mathbf{M}$ is strictly RCDD, it is invertible by Lemma 4 . Thus, let $y$ be the vector satisfying $\mathbf{M} y=b$. By Corollary 20, we can compute, with high probability and in $O\left(\mathcal{T}_{\text {solve }} \log \frac{1}{\alpha \epsilon r}\right)=O\left(\mathcal{T}_{\text {solve }} \log \left(\frac{\epsilon^{2} r^{2}}{\left\|\mathbf{A}^{-1}\right\|_{2}^{2} \cdot u^{2}}\right)^{-1}\right)$ time, a vector $x^{\prime}$ satisfying

$$
\left\|x^{\prime}-y\right\|_{2}\left\|x^{\prime}-y\right\|_{\mathbf{D}} \leq \frac{\alpha \epsilon r}{2 \alpha}\|b\|_{\mathbf{D}^{-1}} \Longrightarrow\left\|x^{\prime}-y\right\|_{2} \leq \frac{\epsilon}{2}\|b\|_{2} .
$$


Let $x$ be the solution to $\mathbf{A} x=b$. Then we have

$\|\mathbf{A} x-\mathbf{A} y\|_{2}=\|b-(b-\alpha \mathbf{D} y)\|_{2}=\alpha\|\mathbf{D} y\|_{2} \leq \alpha \cdot u \cdot\|y\|_{2}=\alpha \cdot u \cdot\left\|\mathbf{M}^{-1} b\right\|_{2} \leq \alpha \cdot u \cdot\left\|\mathbf{M}^{-1}\right\|_{2} \cdot\|b\|_{2}$.

We can bound $\left\|\mathbf{M}^{-1}\right\|_{2}$ by

$$
\begin{aligned}
\left\|\mathbf{M}^{-1}\right\|_{2} & =\left(\min _{v} \frac{\|\mathbf{M} v\|_{2}}{\|v\|_{2}}\right)^{-1}=\left(\min _{v} \frac{\|v\|_{2} \cdot\|\mathbf{M} v\|_{2}}{\|v\|_{2}^{2}}\right)^{-1} \\
& \leq\left(\min _{v} \frac{v^{\top}[\alpha \mathbf{D}+\mathbf{A}] v}{\|v\|_{2}^{2}}\right)^{-1} \leq\left(\alpha r+\lambda_{\min }\left(\mathbf{A}+\mathbf{A}^{\top}\right)\right)^{-1} \leq 1 /(\alpha r) .
\end{aligned}
$$

Substituting this into Lemma 38, we get a bound that doesn't depend on $\alpha$.

$$
\left\|\mathbf{M}^{-1}\right\|_{2} \leq\left(1+\alpha \cdot\left\|\mathbf{M}^{-1}\right\|_{2} \cdot\|\mathbf{D}\|_{2}\right) \cdot\left\|\mathbf{A}^{-1}\right\|_{2} \leq\left(1+\|\mathbf{D}\|_{2} / r\right) \cdot\left\|\mathbf{A}^{-1}\right\|_{2} \leq 2(u / r) \cdot\left\|\mathbf{A}^{-1}\right\|_{2}
$$

Thus, by Lemma 60,

$$
\|x-y\|_{2} \leq \alpha \cdot\left\|\mathbf{A}^{-1}\right\|_{2} \cdot u \cdot\left\|\mathbf{M}^{-1}\right\|_{2} \cdot\|b\|_{2} \leq \alpha \cdot\left\|\mathbf{A}^{-1}\right\|_{2}^{2} \cdot\left(u^{2} / r\right) \cdot\|b\|_{2}=\frac{\epsilon}{2}\|b\|_{2} .
$$

By the triangle inequality, $\left\|x^{\prime}-x\right\|_{2} \leq \epsilon\|b\|_{2}$. Replacing $\epsilon$ with $\epsilon /\|\mathbf{A}\|_{2}$ completes the proof.

We now show that we can approximate $\mathcal{L}^{+} b$ efficiently, even when $b$ is not in the image of $\mathcal{L}$.

Theorem 40. (Laplacian pseudoinverse solver)Let $\mathcal{L}=\mathbf{D}-\mathbf{A}^{\top}$ be an $n \times n$ directed Laplacian matrix of the directed, strongly connected graph $G$. Let $x=\mathcal{L}^{+} b$ where $b \in \mathcal{R}^{n}$. Then for any $0<\epsilon \leq 1$, one can compute, with high probability and in time

$$
O\left(\mathcal{T}_{\text {solve }} \log ^{2}\left(\frac{n \cdot \kappa(\mathbf{D}) \cdot \kappa(\mathcal{L})}{\epsilon}\right)\right)
$$

a vector $x^{\prime}$ satisfying $\left\|x^{\prime}-x\right\|_{2} \leq \epsilon\|x\|_{2}$.

In order to prove this, we prove Theorem 41 which says that one can do this if one has a reasonable upper bound on $\kappa(\mathcal{L})$ (or equivalently, $t_{\text {mix }}$ ). Theorem 40 then immediately follows from the fact that we can, in fact, compute good upper bounds quickly via Theorem 33.

Theorem 41. (Conditional Laplacian solver) Let $\mathcal{L}=\mathbf{D}-\mathbf{A}^{\top}$ be an $n \times n$ directed Laplacian associated with a strongly connected directed graph $G$. Let

$$
\mathcal{R}(\epsilon) \leq O\left(\mathcal{T}_{\text {solve }} \log ^{2}\left(\frac{n \cdot M}{\epsilon}\right)\right)
$$

be the cost of computing an $\epsilon$-approximation to the stationary distribution of $\mathcal{L}$ in the sense of Theorem 33, where $M$ is a known upper bound on $\left\|(\mathbf{I}-\mathbf{W})^{+}\right\|_{2}$, where $\mathbf{W}=\mathbf{A}^{\top} \mathbf{D}^{-1}$ is the associated random walk matrix. Let $x=\mathcal{L}^{+} b$, where $b \in \mathcal{R}^{n}$. Then for any $0<\epsilon \leq 1$, one can compute, with high probability and in time

$$
O\left(\mathcal{T}_{\text {solve }} \log ^{2}\left(\frac{n \cdot \kappa(\mathbf{D}) \cdot M}{\epsilon}\right)+\mathcal{R}\left(\frac{\epsilon}{n \cdot \kappa(\mathbf{D})}\right)\right) \leq O\left(\mathcal{T}_{\text {solve }} \log ^{2}\left(\frac{n \cdot \kappa(\mathbf{D}) \cdot M}{\epsilon}\right)\right)
$$

a vector $x^{\prime}$ satisfying $\left\|x^{\prime}-x\right\|_{2} \leq \epsilon\|x\|_{2}$.

Furthermore, all the intermediate Eulerian Laplacian solves required to produce the approximate solution involve only $\mathbf{R}$ for which $\kappa\left(\mathbf{R}+\mathbf{R}^{\top}\right), \kappa(\operatorname{diag}(\mathbf{R})) \leq(n \kappa(\mathbf{D}) M / \epsilon)^{O(1)}$. 
Proof. Note that since we wish to compute $x=\mathcal{L}^{+} b$, we can initially project $b$ onto $\operatorname{im}(\mathcal{L})=$ $\operatorname{span}(\overrightarrow{1})^{\perp}$. Thus, we will assume for the rest of the proof that $b$ is in the image of $\mathcal{L}$.

We solve the equivalent linear system $(\mathbf{I}-\mathbf{W}) z=b$, where $\mathbf{I}-\mathbf{W}=\mathcal{L} \mathbf{D}^{-1}$, to within accuracy $\epsilon^{\prime}=\epsilon / \kappa(\mathbf{D})$, in the sense that the solution $z^{\prime}$ we obtain satisfies $\left\|z^{\prime}-z\right\|_{2} \leq \epsilon^{\prime} \cdot\|z\|_{2}$. At the end, we return the solution $x^{\prime}=\mathbf{D}^{-1} z^{\prime}$. The guarantee we provide on $z^{\prime}$ is equivalent to $\left\|\mathbf{D}^{-1} x^{\prime}-\mathbf{D}^{-1} x\right\|_{2} \leq$ $\epsilon^{\prime} \cdot\left\|\mathbf{D}^{-1} x\right\|_{2}$, and thus $\left\|\mathbf{D}^{-1}\left(x^{\prime}-x\right)\right\|_{2} \leq \epsilon^{\prime} \cdot\left\|\mathbf{D}^{-1}\right\|_{2}\|x\|_{2}$. We then know that $\left\|x^{\prime}-x\right\|_{2} \leq(\epsilon / \kappa(\mathbf{D}))$. $\kappa(\mathbf{D}) \cdot\|x\|_{2} \leq \epsilon\|x\|_{2}$.

In order to solve the system in $\mathbf{I}-\mathbf{W}$, we set $\mathbf{M}=\alpha \mathbf{I}+(\mathbf{I}-\mathbf{W})$, for $\alpha=\epsilon^{\prime} /(6 M \sqrt{n})$, and find an approximate solution to the system involving $\mathbf{M}$.

First we show that, having set $\alpha$ sufficiently small, the solution to the system in $\mathbf{M}$ is not very far from the true solution $z$. Letting $\Pi$ denote the projection onto the subspace orthogonal to the kernel of $\mathbf{I}-\mathbf{W}$, we upper bound $\left\|\Pi\left(z-\mathbf{M}^{-1} b\right)\right\|_{2}$. First, using the fact that $\mathbf{M}$ is invertible, we notice that $\left\|\mathbf{M}^{-1}\right\|_{2} \leq \max _{x:\|x\|_{2}=1} \frac{1}{\|\mathbf{M} x\|_{2}} \leq \max _{x:\|x\|_{2}=1} \frac{\sqrt{n}}{\|\mathbf{M} x\|_{1}}$. Since $\mathbf{M}$ is $\alpha$-RCDD we have $\|\mathbf{M} x\|_{1} \geq \alpha\|x\|_{1}$. Therefore $\left\|\mathbf{M}^{-1}\right\|_{2} \leq \max _{x:\|x\|_{2}=1} \frac{\sqrt{n}}{\alpha\|x\|_{1}} \leq \sqrt{n} / \alpha$. By Lemma 38, we have $\left\|\mathbf{M}^{-1} b\right\|_{2} \leq\left(1+\alpha\left\|\mathbf{M}^{-1}\right\|_{2}\right) \cdot\left\|(\mathbf{I}-\mathbf{W})^{+} b\right\|_{2}$, and therefore

$$
\left\|\mathbf{M}^{-1} b\right\|_{2} \leq 2 \sqrt{n}\|z\|_{2} \text {. }
$$

Thus we can bound

$$
\begin{aligned}
\left\|\Pi\left(z-\mathbf{M}^{-1} b\right)\right\|_{2} & =\left\|(\mathbf{I}-\mathbf{W})^{+}(\mathbf{I}-\mathbf{W})\left(z-\mathbf{M}^{-1} b\right)\right\|_{2} \leq\left\|(\mathbf{I}-\mathbf{W})^{+}\right\|_{2}\left\|b-(\mathbf{M}-\alpha \mathbf{I}) \mathbf{M}^{-1} b\right\|_{2} \\
& =\left\|(\mathbf{I}-\mathbf{W})^{+}\right\|_{2} \cdot \alpha\left\|\mathbf{M}^{-1} b\right\|_{2} \leq\left\|(\mathbf{I}-\mathbf{W})^{+}\right\| \alpha \cdot 2 \sqrt{n} \cdot\|z\|_{2} \leq \frac{\epsilon^{\prime}}{3}\|z\|_{2} .
\end{aligned}
$$

Next, we provide a bound on the required accuracy for solving $\mathbf{M}^{-1} b$. Using Theorem 2 , we compute in time $O\left(\left(m+\mathcal{T}_{\text {solve }} \log \frac{n}{\alpha}\right) \cdot \log \frac{n}{\alpha}\right)=O\left(\mathcal{T}_{\text {solve }} \log ^{2} \frac{n M \kappa(\mathbf{D})}{\epsilon}\right)$, a diagonal matrix $\mathbf{S} \succeq \mathbf{0}$ such that $\operatorname{tr}(\mathbf{S})=1$, MS is $\frac{\alpha}{3 n}-\operatorname{RCDD}$, and $\kappa(\mathbf{S}) \leq \frac{60 n^{3}}{\alpha^{2}}$. Letting $\epsilon^{\prime \prime}=\epsilon^{\prime} \cdot \alpha^{3} /\left(120 n^{3.5}\right)$, and using Corollary 20, we can compute, with high probability and in $O\left(\mathcal{T}_{\text {solve }} \log \frac{1}{\epsilon^{\prime \prime}}\right)=O\left(\mathcal{T}_{\text {solve }} \log \frac{\kappa(\mathbf{D})}{\epsilon}\right)$ time, a vector $x^{\prime}$ satisfying

$$
\left\|x^{\prime}-(\mathbf{M S})^{-1} b\right\|_{(1+\alpha) \mathbf{S}} \leq \frac{\epsilon^{\prime \prime}}{\alpha}\|b\|_{(1+\alpha)^{-1} \mathbf{S}^{-1}}
$$

with high probability, and equivalently:

$$
(1+\alpha)^{1 / 2}\left\|\mathbf{S} x^{\prime}-\mathbf{M}^{-1} b\right\|_{\mathbf{S}^{-1}} \leq \frac{\epsilon^{\prime \prime}}{\alpha(1+\alpha)^{1 / 2}}\|b\|_{\mathbf{S}^{-1}}
$$

Plugging the bound on $\kappa(\mathbf{S})$, this yields:

$$
\left\|\mathbf{S} x^{\prime}-\mathbf{M}^{-1} b\right\|_{2} \leq \frac{\epsilon^{\prime \prime} \kappa(\mathbf{S})}{\alpha(1+\alpha)}\|b\|_{2} \leq \frac{60 n^{3} \epsilon^{\prime \prime}}{\alpha^{3}(1+\alpha)}\|b\|_{2} .
$$

Since $b$ is in the image of $\mathcal{L}$, therefore in the image of $\mathbf{I}-\mathbf{W}$, and using $\|\mathbf{I}-\mathbf{W}\|_{2} \leq 2 \sqrt{n}$, we obtain:

$$
\left\|\mathbf{S} x^{\prime}-\mathbf{M}^{-1} b\right\|_{2} \leq \frac{60 n^{3} \epsilon^{\prime \prime}}{\alpha^{3}(1+\alpha)}\left\|(\mathbf{I}-\mathbf{W})(\mathbf{I}-\mathbf{W})^{+} b\right\|_{2} \leq \frac{120 n^{3.5} \epsilon^{\prime \prime}}{\alpha^{3}(1+\alpha)}\left\|(\mathbf{I}-\mathbf{W})^{+} b\right\|_{2} \leq \frac{\epsilon^{\prime}}{3}\|z\|_{2}(\pi
$$

Using triangle inequality we then obtain that

$$
\left\|\Pi\left(\mathbf{S} x^{\prime}-z\right)\right\|_{2} \leq\left\|\Pi\left(\mathbf{S} x^{\prime}-\mathbf{M}^{-1} b\right)\right\|_{2}+\left\|\Pi\left(z-\mathbf{M}^{-1} b\right)\right\|_{2} \leq \frac{2 \epsilon^{\prime}}{3}\|z\|_{2} .
$$


So, if we ignore the kernel of $\mathbf{I}-\mathbf{W}$, our solution $\mathbf{S} x^{\prime}$ is close to $z$. The rest of the proof is devoted to showing how to project off something approximating the kernel of $\mathbf{I}-\mathbf{W}$ in order to obtain a vector that unconditionally approximates $z=(\mathbf{I}-\mathbf{W})^{+} b$. Using Theorem 33, we compute in time $\mathcal{R}\left(\epsilon^{\prime} /(32 n)\right)=\mathcal{R}(\epsilon /(32 n \cdot \kappa(\mathbf{D})))$, an approximate stationary vector $s^{\prime}$ satisfying $\left\|s^{\prime}-s^{*}\right\|_{2} \leq \frac{\epsilon^{\prime}}{32 n}$, where $s^{*}$ is the true stationary. We will prove that if we approximate the true stationary sufficiently well, then the approximate projection $\Pi^{\prime} \mathbf{S} x^{\prime}$, where $\Pi^{\prime}=\mathbf{I}-\frac{s^{\prime}}{\left\|s^{\prime}\right\|_{2}}\left(\frac{s^{\prime}}{\left\|s^{\prime}\right\|_{2}}\right)^{\top}$, is unconditionally close to $z$.

First we bound

$$
\begin{aligned}
\left\|\Pi-\Pi^{\prime}\right\|_{2} & =\left\|\left(\mathbf{I}-\frac{s^{*}}{\left\|s^{*}\right\|_{2}}\left(\frac{s^{*}}{\left\|s^{*}\right\|_{2}}\right)^{\top}\right)-\left(\mathbf{I}-\frac{s^{\prime}}{\left\|s^{\prime}\right\|_{2}}\left(\frac{s^{\prime}}{\left\|s^{\prime}\right\|_{2}}\right)^{\top}\right)\right\|_{2} \\
& =\left\|\frac{s^{*}}{\left\|s^{*}\right\|_{2}}\left(\frac{s^{*}}{\left\|s^{*}\right\|_{2}}\right)^{\top}-\frac{s^{\prime}}{\left\|s^{\prime}\right\|_{2}}\left(\frac{s^{\prime}}{\left\|s^{\prime}\right\|_{2}}\right)^{\top}\right\|_{2} \cdot
\end{aligned}
$$

Using Lemma 37, we see that $\left\|\frac{s^{*}}{\left\|s^{*}\right\|_{2}}-\frac{s^{\prime}}{\left\|s^{\prime}\right\|_{2}}\right\|_{2} \leq 2 \cdot \frac{\left\|s^{\prime}-s^{*}\right\|_{2}}{\left\|s^{*}\right\|_{2}} \leq \frac{\epsilon^{\prime}}{16 n} \cdot \sqrt{n}$. Therefore we can use this, together with triangle inequality, to prove that for any vector $y$ :

$$
\begin{aligned}
\left\|\left(\Pi-\Pi^{\prime}\right) y\right\|_{2} & =\left\|\frac{s^{*}}{\left\|s^{*}\right\|_{2}} \cdot \frac{\left(s^{*}\right)^{\top} y}{\left\|s^{*}\right\|_{2}}-\frac{s^{\prime}}{\left\|s^{\prime}\right\|_{2}} \cdot \frac{\left(s^{\prime}\right)^{\top} y}{\left\|s^{\prime}\right\|_{2}}\right\|_{2} \\
& \leq\left\|\frac{s^{\prime}}{\left\|s^{\prime}\right\|_{2}} \cdot\left(\frac{s^{\prime \top} y}{\left\|s^{\prime}\right\|_{2}}-\frac{\left(s^{*}\right)^{\top} y}{\left\|s^{*}\right\|_{2}}\right)\right\|_{2}+\left\|\left(\frac{s^{\prime}}{\left\|s^{\prime}\right\|_{2}}-\frac{s^{*}}{\left\|s^{*}\right\|_{2}}\right) \cdot \frac{\left(s^{*}\right)^{\top} y}{\left\|s^{*}\right\|_{2}}\right\|_{2} \\
& \leq 2\left\|\frac{s^{*}}{\left\|s^{*}\right\|_{2}}-\frac{s^{\prime}}{\left\|s^{\prime}\right\|_{2}}\right\|_{2}\|y\|_{2} \leq \frac{\epsilon^{\prime}}{8 \sqrt{n}}\|y\|_{2} .
\end{aligned}
$$

Therefore, using this together with (7.2) and (7.1), we obtain

$$
\begin{aligned}
\left\|\Pi^{\prime} \mathbf{S} x^{\prime}-\Pi \mathbf{S} x^{\prime}\right\|_{2} & \leq \frac{\epsilon^{\prime}}{8 \sqrt{n}}\left\|\mathbf{S} x^{\prime}\right\|_{2} \leq \frac{\epsilon^{\prime}}{8 \sqrt{n}}\left(\left\|\mathbf{S} x^{\prime}-\mathbf{M}^{-1} b\right\|_{2}+\left\|\mathbf{M}^{-1} b\right\|_{2}\right) \\
& \leq \frac{\epsilon^{\prime}}{8 \sqrt{n}} \cdot\left(\frac{\epsilon^{\prime}}{3}\|z\|_{2}+2 \sqrt{n}\|z\|_{2}\right) \leq \frac{\epsilon^{\prime}}{3}\|z\|_{2} .
\end{aligned}
$$

Using this together with (7.3), and applying the triangle inequality, we obtain that $\left\|\Pi^{\prime} \mathbf{S} x^{\prime}-z\right\|_{2} \leq$ $\epsilon^{\prime}\|z\|_{2}$.

Finally, as we can see from Theorem 2 , all the intermediate solves involve $(\alpha / 3 n)$-RCDD matrices for which the condition number of their diagonals is bounded by $(n / \alpha)^{O(1)}$. Using Lemma 64, we see that applying the reduction from Theorem 19 to these matrices only outputs matrices whose symmetrizations have condition number bounded by $4 n^{3} \cdot 3 n / \alpha \cdot(n / \alpha)^{O(1)}=(n \kappa(\mathbf{D}) M / \epsilon)^{O(1)}$.

We also note that the first part of the proof of Theorem 41 also gives a solver for a more general class of linear systems-arbitrary row- or column-diagonally dominant systems. This includes directed Laplacians for graphs that are not strongly connected. As the null space of such matrices are more intricate, we give the following guarantee in terms of the $\ell_{2}$ norm of the matrix-vector product.

Theorem 42. (Diagonally dominant solver)Let $\mathbf{M}$ be an arbitrary $n \times n$ column-diagonally-dominant or row-diagonally-dominant matrix with diagonal $\mathbf{D}$. Let $b \in \operatorname{im}(\mathbf{M})$. Then for any $0<\epsilon \leq 1$, one can compute, with high probability and in time 


$$
O\left(\mathcal{T}_{\text {solve }} \log ^{2}\left(\frac{n \cdot \kappa(\mathbf{D}) \cdot \kappa(\mathbf{M})}{\epsilon}\right)\right)
$$

a vector $x^{\prime}$ satisfying $\left\|\mathbf{M} x^{\prime}-b\right\|_{2} \leq \epsilon\|b\|_{2}$.

Furthermore, all the intermediate Eulerian Laplacian solves required to produce the approximate solution involve only matrices $\mathbf{R}$ for which $\kappa\left(\mathbf{R}+\mathbf{R}^{\top}\right), \kappa(\operatorname{diag}(\mathbf{R})) \leq(n \kappa(\mathbf{D}) \kappa(\mathbf{M}) / \epsilon)^{O(1)}$.

Proof. The algorithm and proof are essentially the same as the first part of that of Theorem 41.

First, let us analyze the CDD case. Suppose $K$ is a known upper bound on $\kappa(\mathbf{M})$. We rescale $\mathbf{M}$ by the diagonal, and solve for $\widehat{\mathbf{M}}=\mathbf{M D}^{-1}$ to within accuracy $\epsilon / \kappa(\mathbf{D})$. In order to do so, we consider the matrix $\tilde{\mathbf{M}}$ with a slightly increased diagonal

$$
\tilde{\mathbf{M}}=\frac{1}{\alpha} \mathbf{I}+\widehat{\mathbf{M}}
$$

where $\alpha=\epsilon^{\prime} /(6 \kappa(\mathbf{D}) K n) \leq \epsilon /\left(6 \kappa^{2}(\mathbf{D}) K n\right)$. We notice that $\left\|\left(\mathbf{M D}^{-1}\right)^{+}\right\|_{2}=\kappa\left(\mathbf{M D}^{-1}\right) /\left\|\mathbf{M D}^{-1}\right\|_{2}$, and the term in the denominator can be lower bounded by $\left\|\mathbf{M D}^{-1}\right\|_{1} / \sqrt{n}$. But by definition the corresponding norm of the rescaled matrix is at least 1 ; thus $\left\|\left(\mathbf{M D}^{-1}\right)^{+}\right\|_{2} \leq \kappa\left(\mathbf{M D}^{-1}\right) \cdot \sqrt{n} \leq$ $\kappa(\mathbf{D}) \kappa(\mathbf{M}) \sqrt{n} \leq \kappa(\mathbf{D}) K \sqrt{n}$, where for the last inequality we used Lemma 63. So our setting of $\alpha$ corresponds to the what is required for the analysis we have seen in Theorem 41. An approximate rescaling of it to the RCDD case is obtained by considering the diagonal scaling $\mathbf{S}$ of the matrix $\tilde{\mathbf{M}}_{-}$, which has all the off-diagonal entries replaced by negative entries with the same magnitude. With this rescaling, solutions to the system $\tilde{\mathbf{M}} x=b$ can be approximated to high accuracy using Theorem 39. The $\ell_{2}$ norm error guarantees of the solution to $\tilde{\mathbf{M}}^{+} b$ follows via the exact same sequence of inequalities, namely Equations 7.1 and 7.2. The second part involving the projections from the kernel is not necessary since we measure only $\left\|\mathbf{M} x^{\prime}-b\right\|_{2}$.

Finally, in order to eliminate the assumption on $K$, we can simply run the algorithm with $K=2^{2^{0}}, 2^{2^{1}}, \ldots$ and test if the returned solution satisfies the required bound. This adds at most a constant overhead, since the running time of the algorithm doubles between iterations, therefore it is dominated by the one involving a value of $K$ that is within a polynomial factor from the true $\kappa(\mathbf{M})$.

The RDD case is treated similarly. The difference is that the initial rescaling is done in order to produce $\widehat{\mathbf{M}}=\mathbf{D}^{-1} \mathbf{M}$, and that the rescaling is computed with respect to $\tilde{\mathbf{M}}^{\top}$.

Again, as seen in the statement of Theorem 2, all the intermediate solves involve $(\alpha / 3 n)$-RCDD matrices for which the condition number of their diagonals is bounded by $(n / \alpha)^{O(1)}$. Just like before, we see that by Lemma 64, plugging these into the reduction from Theorem 19 produces matrices with condition number bounded by $(n / \alpha)^{O(1)}=(n \kappa(\mathbf{D}) \kappa(\mathbf{M}) / \epsilon)^{O(1)}$.

We believe a guarantee more similar to Theorem 41 can be obtained, but would require methods that better take the null space of these matrices into account. One plausible approach for directed Laplacians is to topologically sort the strongly connected components, and then perform backsubstitutions.

\subsection{Hitting and Commute Times}

In this section we show how to compute hitting times and commute times for a random walk on a directed graph by solving only $O(1)$ linear systems involving the associated directed Laplacian. We first formally define hitting and commute times and then we provide Lemma 45 which yields a short formula of these quantities in terms of the directed Laplacian pseudoinverse. This suggests a 
natural algorithm which formally analyze in the main result of this section, Theorem 46 to compute them, and then formally analyze the error required by this Laplacian solve.

Definition 43. The hitting time $H_{u v}$ denotes the expected number of steps a random walk starting at $u$ takes until first reaching $v$.

Definition 44. The commute time $C_{u v}$ denotes the expected number of steps for a random walk starting at $u$ to reach $v$, then return to $u$. In other words, $C_{u v}=H_{u v}+H_{v u}$.

These quantities can each be computed using the below identities, which hold for Markov chains whose associated graph is strongly connected.

Lemma 45. Let $\mathbf{W} \in \mathcal{R}_{\geq 0}^{n \times n}$ be a random walk matrix associated to a strongly connected graph. Let $s$ be its stationary distribution. The hitting time $H_{u v}$ satisfies

$$
H_{u v}=\left(\overrightarrow{1}-\overrightarrow{1}_{v} \cdot \frac{1}{s_{v}}\right)^{\top}(\mathbf{I}-\mathbf{W})^{+}\left(\overrightarrow{1}_{u}-\overrightarrow{1}_{v}\right) .
$$

We prove this identity in Section C.1. It suggests the following natural algorithm for computing hitting times.

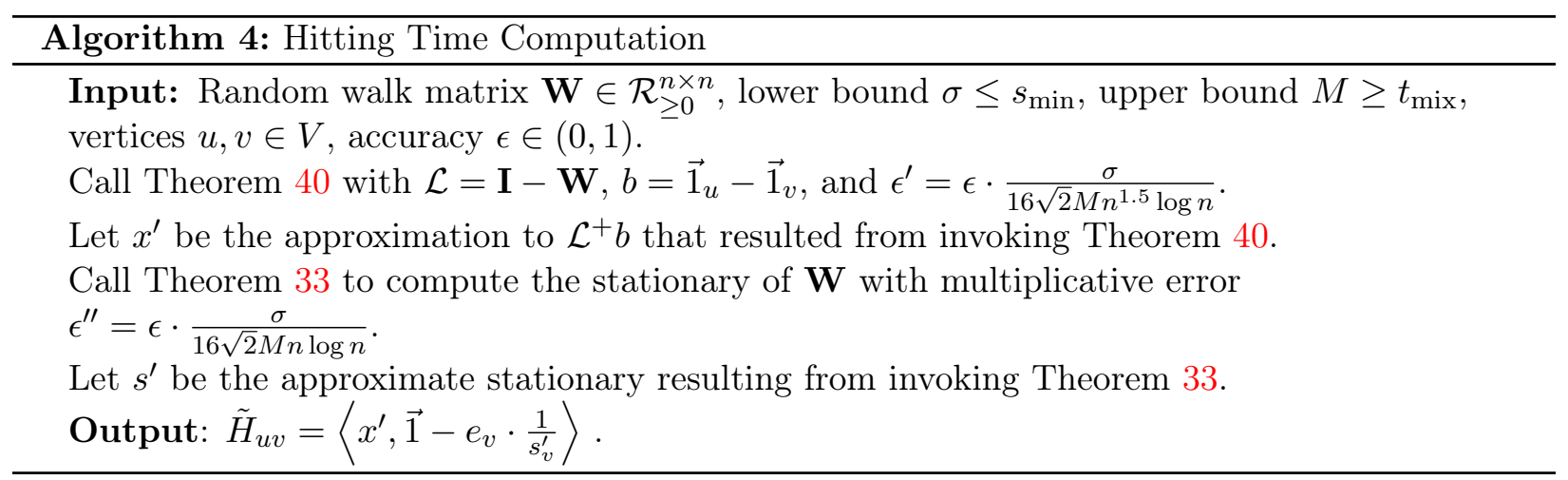

Theorem 46. (Hitting Time Computation) Let $\mathbf{W} \in \mathcal{R}_{\geq 0}^{n \times n}$ be a random walk matrix with $m$ nonzero entries, associated with a strongly connected graph. Let $\sigma \leq s_{\min }$ be a lower bound on its minimum stationary probability, let $M \geq t_{\operatorname{mix}}$ be an upper bound on its mixing time, and let $u, v \in\{1, \ldots, n\}$. Then for any $\epsilon \in(0,1)$, Algorithm \& computes $\tilde{H}_{u v}$ such that $\left|\tilde{H}_{u v}-H_{u v}\right| \leq \epsilon$ in time $O\left(\mathcal{T}_{\text {solve }} \log ^{2}\left(\frac{M n}{\epsilon \sigma}\right)\right)$, with high probability in $n$.

Proof. First we bound the running time. Theorem 40 gives that approximates $\mathcal{L}^{+} b$ takes time $O\left(\mathcal{T}_{\text {solve }} \log ^{2}\left(\frac{n \kappa(\mathbf{S}) \kappa(\mathbf{I}-\mathbf{W})}{\epsilon^{\prime}}\right)\right)=O\left(\mathcal{T}_{\text {solve }} \log ^{2}\left(\frac{M n}{\epsilon \sigma}\right)\right)$. The call to Theorem 33 produces an approximate stationary in time $O\left(\mathcal{T}_{\text {solve }} \log ^{2}\left(\frac{n M \kappa(\mathbf{S})}{\epsilon^{\prime \prime}}\right)\right)=O\left(\mathcal{T}_{\text {solve }} \log ^{2}\left(\frac{n M}{\epsilon \sigma}\right)\right)$. The desired bound then follows from all the other operations taking $O(n)$ time, which is sublinear in $\mathcal{T}_{\text {solve }}$.

We write the approximation error $\left|\tilde{H}_{u v}-H_{u v}\right|$ explicitly, and expand it by applying the triangle inequality. Letting $x=(\mathbf{I}-\mathbf{W})^{+}\left(\overrightarrow{1}_{u}-\overrightarrow{1}_{v}\right)$ we obtain:

$$
\begin{array}{r}
\quad\left|\tilde{H}_{u v}-H_{u v}\right|=\left|\left\langle x^{\prime}, \overrightarrow{1}-\overrightarrow{1}_{v} \cdot \frac{1}{s_{v}^{\prime}}\right\rangle-\left\langle x, \overrightarrow{1}-\overrightarrow{1}_{v} \cdot \frac{1}{s_{v}}\right\rangle\right| \leq\left(\sum_{i}\left|x_{i}^{\prime}-x_{i}\right|\right)+\left|\frac{x_{v}^{\prime}}{s_{v}^{\prime}}-\frac{x_{v}}{s_{v}}\right| \\
\leq\left\|x^{\prime}-x\right\|_{1}+\left|\frac{x_{v}^{\prime}-x_{v}}{s_{v}^{\prime}}\right|+\left|\frac{x_{v}}{s_{v}^{\prime}}-\frac{x_{v}}{s_{v}}\right| \leq\left\|x^{\prime}-x\right\|_{2} \sqrt{n} \cdot\left(1+\frac{1}{s_{v}^{\prime}}\right)+\left|x_{v}\right|\left(\frac{1}{s_{v}^{\prime}}-\frac{1}{s_{v}}\right) .
\end{array}
$$


The call to Theorem 40 returns $x^{\prime}$ satisfying $\left\|x-x^{\prime}\right\|_{2} \leq \epsilon^{\prime}\|x\|_{2}$. Also, the call to Theorem 33 returns $s^{\prime}$ satisfying $\left|s_{v}^{\prime}-s_{v}\right| \leq \epsilon^{\prime \prime} \cdot s_{v}$, so $\left|\frac{1}{s_{v}^{\prime}}-\frac{1}{s_{v}}\right| \leq \frac{2 \epsilon}{s_{v}}$. Therefore, since $\left|x_{v}\right| \leq\|x\|_{2}$,

$$
\left|\tilde{H}_{u v}-H_{u v}\right| \leq\|x\|_{2} \cdot\left(\epsilon^{\prime} \sqrt{n} \cdot\left(1+\frac{1}{\sigma}\right)+\frac{2 \epsilon^{\prime \prime}}{\sigma}\right) .
$$

Plugging in $\|x\|_{2} \leq\left\|(\mathbf{I}-\mathbf{W})^{+}\right\|_{2}\left\|\overrightarrow{1}_{u}-\overrightarrow{1}_{v}\right\|_{2}$, we see that error satisfies

$$
\begin{aligned}
\left|\tilde{H}_{u v}-H_{u v}\right| & \leq\left\|(\mathbf{I}-\mathbf{W})^{+}\right\|_{2} \sqrt{2} \cdot\left(\epsilon^{\prime} \sqrt{n} \cdot\left(1+\frac{1}{\sigma}\right)+\frac{2 \epsilon^{\prime \prime}}{\sigma}\right) \\
& \leq M \cdot 4 n \log n \cdot \sqrt{2} \cdot\left(\epsilon^{\prime} \sqrt{n} \cdot\left(1+\frac{1}{\sigma}\right)+\epsilon^{\prime \prime} \frac{2}{\sigma}\right),
\end{aligned}
$$

where, for the last inequality, we used the bound on $\left\|(\mathbf{I}-\mathbf{W})^{+}\right\|_{2}$ from Theorem 21. Plugging in the values for $\epsilon^{\prime}$ and $\epsilon^{\prime \prime}$ yields the result.

Combining the formulas for hitting times also leads to an expression for commute time, $C_{u v}=$ $H_{u v}+H_{v u}$. It can be computed by simply invoking Theorem 46 twice.

\subsection{Escape probabilities}

In this section we derive a simple formula for escape probabilities in a Markov chain, and show how to compute them using our directed Laplacian solver. We first define escape probabilities, then we show how they can be computed using our directed Laplacian solver.

Definition 47. Let $u$ and $v$ be two different vertices in a Markov chain. The escape probability $p_{w}$ denotes the probability that starting at vertex $w$, a random walk reaches $u$ before first reaching $v .^{5}$

Lemma 48. Let $\mathbf{W} \in \mathcal{R}_{\geq 0}^{n \times n}$ be a random walk matrix associated with a strongly connected graph. Let $s$ be its stationary distribution, and let $u, v$ be two of its vertices. Let $p$ be the vector of escape probabilities, where $p_{w}$ represents the probability that a random walk starting at $w$ reaches $u$ before $v$. Then

$$
p=\beta\left(\alpha s+(\mathbf{I}-\mathbf{W})^{+}\left(\overrightarrow{1}_{u}-\overrightarrow{1}_{v}\right)\right)
$$

where $\alpha=-e_{v}^{\top} \mathbf{S}^{-1}(\mathbf{I}-\mathbf{W})^{+}\left(\overrightarrow{1}_{u}-\overrightarrow{1}_{v}\right)$ and $\beta=\frac{1}{s_{u}\left(\overrightarrow{1}_{u}-\overrightarrow{1}_{v}\right)^{\top} \mathbf{S}^{-1}(\mathbf{I}-\mathbf{W})^{+}\left(\overrightarrow{1}_{u}-\overrightarrow{1}_{v}\right)}$. Furthermore,

$$
|\alpha| \leq \frac{t_{\mathrm{mix}}}{s_{\min }} \cdot 8 \sqrt{n} \log n
$$

and

$$
\frac{1}{t_{\text {mix }} \cdot 8 \sqrt{n} \log n} \leq \beta \leq \frac{1}{s_{\min }} .
$$

The proof can be found in Section C.3. Using it, we can compute escape probabilities in time $\tilde{O}\left(\mathcal{T}_{\text {solve }}\right)$ using the algorithm described below.

\footnotetext{
${ }^{5}$ One should note that escape probabilities can similarly be defined with respect to sets of vertices, not just singleton vertices. In particular, one can define escape probabilities just like above with respect to two disjoint sets $S$ and $T$. However, this instance reduces to the one above simply by contracting the sets into single vertices.
} 


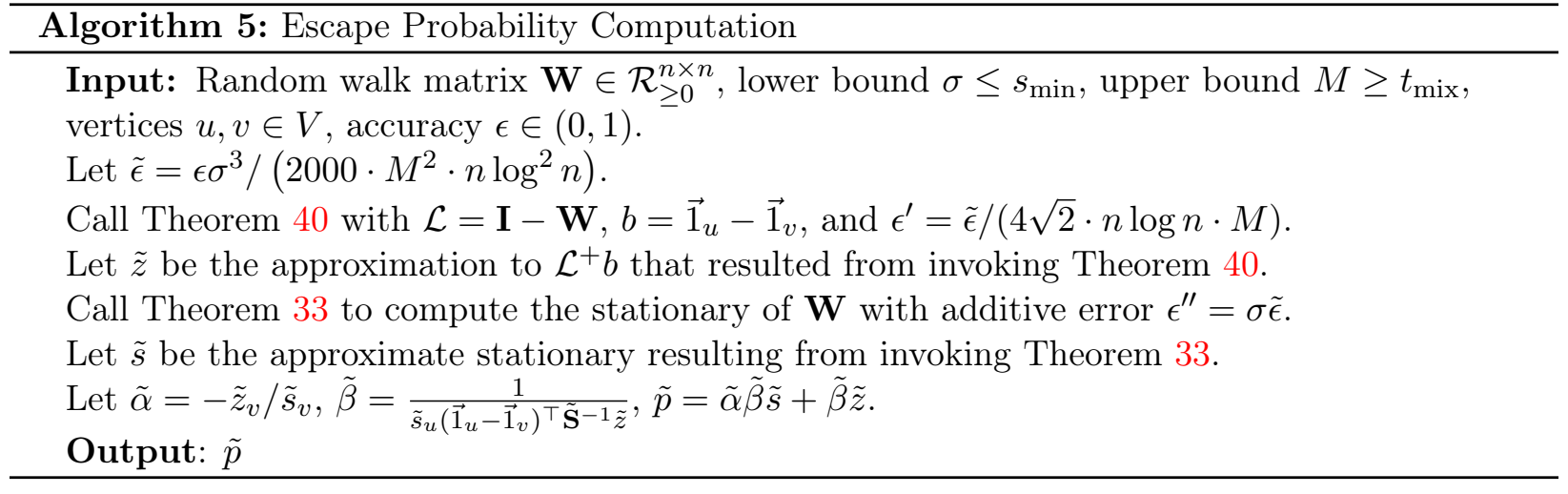

Theorem 49. (Escape Probability Computation) Let $\mathbf{W} \in \mathcal{R}_{\geq 0}^{n \times n}$ be a random walk matrix with $m$ non-zero entries, i.e. $\mathbf{I}-\mathbf{W}$ is a directed Laplacian, let $\sigma \leq s_{\min }$ be a lower bound on the minimum stationary probability, $M \geq t_{\text {mix }}$ an upper bound on the mixing time, and let $u, v \in\{1, \ldots, n\}$. Then for any $\epsilon \in(0,1)$, Algorithm 5 computes a vector $\tilde{p}$ such that $\left|\tilde{p}_{i}-p_{i}\right| \leq \epsilon$ for all $i$ with high probability in $n$ in time $O\left(\mathcal{T}_{\text {solve }} \log ^{2}\left(\frac{n M}{\epsilon \sigma}\right)\right)$, where $p$ is the vector of escape probabilities as defined in Definition $4 \%$.

Proof. First we analyze the running time. From the description of the algorithm, we see that the running time is dominated by the calls to Theorem 40, respectively Theorem 33. The first one computes $\tilde{z}$ in time time $O\left(\mathcal{T}_{\text {solve }} \log ^{2}\left(\frac{n \cdot \kappa(s) \cdot \kappa(\mathbf{I}-\mathbf{W})}{\epsilon^{\prime}}\right)\right)=O\left(\mathcal{T}_{\text {solve }} \log ^{2}\left(\frac{n M}{\epsilon \sigma}\right)\right)$. The second one computes $\tilde{s}$ in time $O\left(\mathcal{T}_{\text {solve }} \log ^{2}\left(\frac{n M}{\epsilon^{\prime \prime}}\right)\right)=O\left(\mathcal{T}_{\text {solve }} \log ^{2}\left(\frac{n M}{\epsilon \sigma}\right)\right)$. Hence the bound on running time.

Next, we analyze the error. As seen in Lemma 48, we can write the true escape probability vector as $p=\beta(\alpha s+z)$, where $z=(\mathbf{I}-\mathbf{W})^{+}\left(e_{u}-e_{v}\right)$. Our algorithm uses the approximations to $s$ and $z$ that it has access to in order to obtain $\tilde{\alpha}$ and $\tilde{\beta}$. We will show that these approximate the original values sufficiently well to provide additive guarantees on each element of $\tilde{p}$.

By definition, $\tilde{\epsilon} \leq \epsilon s_{\text {min }}^{3} /\left(2000 \cdot t_{\text {mix }}^{2} \cdot n \log ^{2} n\right)$. In order to provide bounds on the error of the approximation, we will prove that:

1. $\|\tilde{z}-z\|_{\infty} \leq \tilde{\epsilon}$

2. $\left|\tilde{s}_{i}-s_{i}\right| \leq \tilde{\epsilon} s_{i}$ for all $i$

3. $|\alpha-\tilde{\alpha}| \leq \tilde{\epsilon} \cdot 20 \frac{t_{\operatorname{mix}}}{s_{\min }} \cdot \sqrt{n} \log n=\tilde{\epsilon}_{\alpha}$

4. $|\tilde{\beta}-\beta| \leq \frac{18}{s_{\min }} \tilde{\epsilon} \cdot \beta=\tilde{\epsilon}_{\beta} \cdot \beta$.

Indeed, combining them we obtain

$$
\begin{aligned}
\|\tilde{p}-p\|_{\infty} & =\|\tilde{\alpha} \tilde{\beta} \tilde{s}+\tilde{\beta} \tilde{z}-(\alpha \beta s+\beta z)\|_{\infty} \\
& \leq\|\tilde{\alpha} \tilde{\beta} \tilde{s}-\alpha \beta s\|_{\infty}+\|\tilde{\beta} \tilde{z}-\beta z\|_{\infty} \\
& \leq\|\tilde{\beta} \tilde{s}(\tilde{\alpha}-\alpha)\|_{\infty}+\|(\tilde{\beta} \tilde{s}-\beta s) \alpha\|_{\infty}+\|\tilde{\beta}(\tilde{z}-z)\|_{\infty}+\|(\tilde{\beta}-\beta) z\|_{\infty} \\
& \leq|\tilde{\beta}||\tilde{\alpha}-\alpha|\|\tilde{s}\|_{\infty}+|\alpha|\|\tilde{\beta} \tilde{s}-\beta s\|_{\infty}+|\tilde{\beta}|\|\tilde{z}-z\|_{\infty}+(\tilde{\beta}-\beta)\|z\|_{\infty} \\
& \leq\left(1+\tilde{\epsilon}_{\beta}\right) \beta \cdot \tilde{\epsilon}_{\alpha} \cdot(1+\tilde{\epsilon})+|\alpha| \cdot 2\left(\tilde{\epsilon}+\tilde{\epsilon}_{\beta}\right)\|\beta s\|_{\infty}+\left(1+\tilde{\epsilon}_{\beta}\right) \beta \tilde{\epsilon}+\tilde{\epsilon}_{\beta} \beta\left\|_{z}\right\|_{\infty} .
\end{aligned}
$$


Next, we use the fact that $z=\frac{1}{\beta} p-\alpha s$, hence $\|z\|_{\infty} \leq \frac{1}{\beta}\|p\|_{\infty}+|\alpha|\|s\|_{\infty} \leq \frac{1}{\beta}+|\alpha|$. Combining with the previous bound we obtain

$$
\begin{aligned}
\|\tilde{p}-p\|_{\infty} & \leq \tilde{\epsilon}_{\alpha}(1+\tilde{\epsilon})\left(1+\tilde{\epsilon}_{\beta}\right) \beta+2\left(\tilde{\epsilon}+\tilde{\epsilon}_{\beta}\right)|\alpha| \beta+\tilde{\epsilon}\left(1+\tilde{\epsilon}_{\beta}\right) \beta+\tilde{\epsilon}_{\beta} \beta\left(\frac{1}{\beta}+|\alpha|\right) \\
& \leq \tilde{\epsilon}_{\alpha} \cdot 12|\alpha| \beta \\
& \leq \tilde{\epsilon}_{\alpha} \cdot 12 \cdot\left(\frac{t_{\mathrm{mix}}}{s_{\min }} \cdot 8 \sqrt{n} \log _{2} n\right) \cdot \frac{1}{s_{\min }} \\
& \leq \epsilon .
\end{aligned}
$$

Therefore it suffices to prove statements 1-4 from before.

1. Theorem 40 gives us that $\left\|\tilde{z}-(\mathbf{I}-\mathbf{W})^{+}\left(\overrightarrow{1}_{u}-\overrightarrow{1}_{v}\right)\right\|_{2} \leq \epsilon^{\prime}\|x\|_{2} \leq \epsilon^{\prime} \cdot \frac{1}{4 \sqrt{2} \cdot n \log _{2}(n) \cdot M} \cdot\|x\|_{2}$. Therefore $\|\tilde{z}-z\|_{2} \leq \tilde{\epsilon}$.

2. Theorem 33 gives us that $\|\tilde{s}-s\|_{\infty} \leq\|\tilde{s}-s\|_{2} \leq \epsilon^{\prime \prime}$. Hence $\left|\tilde{s}_{i}-s_{i}\right| \leq \frac{\epsilon^{\prime \prime}}{s_{\min }} \cdot s_{i} \leq \tilde{\epsilon} s_{i}$ for all $i$.

3. We write $|\tilde{\alpha}-\alpha|=\left|\frac{\tilde{z}_{v}}{\tilde{s}_{v}}-\frac{z_{v}}{s_{v}}\right|=\left|\frac{s_{v}}{\tilde{s}_{v}} \cdot \frac{\tilde{z}_{v}}{s_{v}}-\frac{z_{v}}{s_{v}}\right| \leq\left|\frac{\tilde{z}_{v}}{s_{v}}-\frac{z_{v}}{s_{v}}\right|+\left|\left(\frac{s_{v}}{\tilde{s}_{v}}-1\right) \cdot \frac{\tilde{z}_{v}}{s_{v}}\right|$. Next, using the additive bound on $\tilde{z}$, respectively the multiplicative bound on $\tilde{s}_{v}$ we see that this is further upper bounded by $\frac{1}{s_{v}}\left|\tilde{z}_{v}-z_{v}\right|+\left|2 \tilde{\epsilon} s_{v} \cdot \frac{\tilde{z}_{v}}{s_{v}}\right| \leq \frac{1}{s_{\min }} \cdot\left|\tilde{z}_{v}-z_{v}\right|+2 \tilde{\epsilon} \cdot \tilde{z}_{v}$. Applying triangle inequality, we see that this is at most $\left|\tilde{z}_{v}-z_{v}\right| \cdot\left(\frac{1}{s_{\min }}+2 \tilde{\epsilon}\right)+2 \tilde{\epsilon} \cdot z_{v}$. Similarly to the proof of Lemma 48 , we bound $z_{v} \leq\left\|(\mathbf{I}-\mathbf{W})^{+}\left(\overrightarrow{1}_{u}-\overrightarrow{1}_{v}\right)\right\|_{1} \leq t_{\text {mix }} \cdot 8 \sqrt{n} \log n$. Therefore $|\tilde{\alpha}-\alpha| \leq$ $\tilde{\epsilon} \cdot \frac{3}{s_{\min }}+\tilde{\epsilon} \cdot t_{\text {mix }} \cdot 16 \sqrt{n} \log n$, which gives our bound.

4. We have $|\tilde{\beta}-\beta|=\left|\frac{1}{\tilde{s}_{u}\left(\overrightarrow{1}_{u}-\overrightarrow{1}_{v}\right)^{\top} \tilde{\mathbf{S}}^{-1} \tilde{z}}-\frac{1}{s_{u}\left(\overrightarrow{1}_{u}-\overrightarrow{1}_{v}\right)^{\top} \mathbf{S}^{-1} z}\right|$. By triangle inequality, we see that this is at most $\left|\left(\frac{1}{\tilde{s}_{u}}-\frac{1}{s_{u}}\right) \cdot \frac{1}{\left(\overrightarrow{1}_{u}-\overrightarrow{1}_{v}\right)^{\top} \mathbf{S}^{-1} z}\right|+\left|\frac{1}{\tilde{s}_{u}} \cdot\left(\frac{1}{\left(\overrightarrow{1}_{u}-\overrightarrow{1}_{v}\right)^{\top} \mathbf{S}^{-1} z}-\frac{1}{\left(\overrightarrow{1}_{u}-\overrightarrow{1}_{v}\right)^{\top} \tilde{\mathbf{S}}^{-1} \tilde{z}}\right)\right|$. Using the multiplicative bound on $\tilde{s}$ we bound it with

$$
\frac{2 \tilde{\epsilon}}{s_{u}} \cdot\left|\frac{1}{\left(\overrightarrow{1}_{u}-\overrightarrow{1}_{v}\right)^{\top} \mathbf{S}^{-1} z}\right|+\frac{1}{(1-\tilde{\epsilon}) s_{u}} \cdot\left|\frac{1}{\left(\overrightarrow{1}_{u}-\overrightarrow{1}_{v}\right)^{\top} \mathbf{S}^{-1} z}-\frac{1}{\left(\overrightarrow{1}_{u}-\overrightarrow{1}_{v}\right)^{\top} \tilde{\mathbf{S}}^{-1} \tilde{z}}\right| .
$$

Now let us bound $\left|\frac{1}{\left(\overrightarrow{1}_{u}-\overrightarrow{1}_{v}\right)^{\top} \mathbf{S}^{-1} z}-\frac{1}{\left(\overrightarrow{1}_{u}-\overrightarrow{1}_{v}\right)^{\top} \tilde{\mathbf{S}}^{-1} \tilde{z}}\right|$. By triangle inequality, it is at most

$$
\left|\frac{1}{\left(\overrightarrow{1}_{u}-\overrightarrow{1}_{v}\right)^{\top} \mathbf{S}^{-1} z}-\frac{1}{\left(\overrightarrow{1}_{u}-\overrightarrow{1}_{v}\right)^{\top} \mathbf{S}^{-1} \tilde{z}}\right|+\left|\frac{1}{\left(\overrightarrow{1}_{u}-\overrightarrow{1}_{v}\right)^{\top} \mathbf{S}^{-1} \tilde{z}}-\frac{1}{\left(\overrightarrow{1}_{u}-\overrightarrow{1}_{v}\right)^{\top} \tilde{\mathbf{S}}^{-1} \tilde{z}}\right| .
$$

Using the multiplicative bound on $\tilde{s}$ we see that this is at most

$$
\left|\frac{1}{\left(\overrightarrow{1}_{u}-\overrightarrow{1}_{v}\right)^{\top} \mathbf{S}^{-1} z}-\frac{1}{\left(\overrightarrow{1}_{u}-\overrightarrow{1}_{v}\right)^{\top} \mathbf{S}^{-1} \tilde{z}}\right|+\left|\frac{2 \tilde{\epsilon}}{\left(\overrightarrow{1}_{u}-\overrightarrow{1}_{v}\right)^{\top} \mathbf{S}^{-1} \tilde{z}}\right|,
$$

which, again, by triangle inequality we bound with

$$
\left|\frac{1}{\left(\overrightarrow{1}_{u}-\overrightarrow{1}_{v}\right)^{\top} \mathbf{S}^{-1} z}-\frac{1}{\left(\overrightarrow{1}_{u}-\overrightarrow{1}_{v}\right)^{\top} \mathbf{S}^{-1} \tilde{z}}\right|+2 \tilde{\epsilon}\left(\left|\frac{1}{\left(\overrightarrow{1}_{u}-\overrightarrow{1}_{v}\right)^{\top} \mathbf{S}^{-1} z}\right|+\left|\frac{1}{\left(\overrightarrow{1}_{u}-\overrightarrow{1}_{v}\right)^{\top} \mathbf{S}^{-1} z}-\frac{1}{\left(\overrightarrow{1}_{u}-\overrightarrow{1}_{v}\right)^{\top} \mathbf{S}^{-1} \tilde{z}}\right|\right) .
$$


Using $\left|\frac{1}{a}-\frac{1}{a+b}\right|=\left|\frac{b}{a(a+b)}\right| \leq 2\left|\frac{b}{a}\right|$, if $a+b \geq 1$, with $a=\left(\overrightarrow{1}_{u}-\overrightarrow{1}_{v}\right)^{\top} \mathbf{S}^{-1} z$ and $b=\left(\overrightarrow{1}_{u}-\right.$ $\left.\overrightarrow{1}_{v}\right)^{\top} \mathbf{S}^{-1}(\tilde{z}-z)$ (which satisfies the conditions since $a \geq 1$ and $|b| \leq\left\|\mathbf{S}^{-1}\left(\overrightarrow{1}_{u}-\overrightarrow{1}_{v}\right)\right\|_{2}\|\tilde{z}-z\|_{2} \leq$ $\left.\frac{\sqrt{2}}{s_{\min }} \cdot \tilde{\epsilon} \leq \frac{1}{2}\right)$ we obtain that the quantity above is at most

$$
\begin{aligned}
& 2\left|\frac{\left(\overrightarrow{1}_{u}-\overrightarrow{1}_{v}\right)^{\top} \mathbf{S}^{-1}(\tilde{z}-z)}{\left(\overrightarrow{1}_{u}-\overrightarrow{1}_{v}\right)^{\top} \mathbf{S}^{-1} z}\right|(1+2 \tilde{\epsilon})+\left|\frac{2 \tilde{\epsilon}}{\left(\overrightarrow{1}_{u}-\overrightarrow{1}_{v}\right)^{\top} \mathbf{S}^{-1} z}\right| \\
= & \frac{1}{\left(\overrightarrow{1}_{u}-\overrightarrow{1}_{v}\right)^{\top} \mathbf{S}^{-1} z} \cdot\left(2\left|\left(\overrightarrow{1}_{u}-\overrightarrow{1}_{v}\right)^{\top} \mathbf{S}^{-1}(\tilde{z}-z)\right|(1+2 \tilde{\epsilon})+2 \tilde{\epsilon}\right) .
\end{aligned}
$$

Applying the Cauchy-Schwarz inequality: $\left|\left(\overrightarrow{1}_{u}-\overrightarrow{1}_{v}\right)^{\top} \mathbf{S}^{-1}(\tilde{z}-z)\right| \leq\left\|\mathbf{S}^{-1}\left(\overrightarrow{1}_{u}-\overrightarrow{1}_{v}\right)^{\top}\right\|_{2}\|\tilde{z}-z\|_{2}$, we see that the quantity is at most

$$
\begin{aligned}
\frac{1}{\left(\overrightarrow{1}_{u}-\overrightarrow{1}_{v}\right)^{\top} \mathbf{S}^{-1} z}\left(2\left\|\mathbf{S}^{-1}\left(\overrightarrow{1}_{u}-\overrightarrow{1}_{v}\right)^{\top}\right\|_{2}\|\tilde{z}-z\|_{2} \cdot(1+2 \tilde{\epsilon})+2 \tilde{\epsilon}\right) & \leq \\
\frac{1}{\left(\overrightarrow{1}_{u}-\overrightarrow{1}_{v}\right)^{\top} \mathbf{S}^{-1} z}\left(\frac{2 \sqrt{2}}{s_{\min }} \cdot \tilde{\epsilon}(1+2 \tilde{\epsilon})+2 \tilde{\epsilon}\right) & \leq \frac{1}{\left(\overrightarrow{1}_{u}-\overrightarrow{1}_{v}\right)^{\top} \mathbf{S}^{-1} z} \cdot \frac{8}{s_{\min }} \tilde{\epsilon}
\end{aligned}
$$

Plugging back into the bound for $|\tilde{\beta}-\beta|$ we obtain that

$$
|\tilde{\beta}-\beta| \leq \frac{1}{s_{u}\left(\overrightarrow{1}_{u}-\overrightarrow{1}_{v}\right)^{\top} \mathbf{S}^{-1} z}\left(2 \tilde{\epsilon}+\frac{1}{(1-\tilde{\epsilon})} \cdot \frac{8}{s_{\min }} \tilde{\epsilon}\right) \leq \beta \cdot \frac{18}{s_{\min }} \tilde{\epsilon}
$$

\subsection{Sketching Commute Times}

In this section, we show that we can efficiently compute an $O\left(\epsilon^{-2} \log n\right) \times n$ matrix that allows us to compute a $(1+\epsilon)$ multiplicative approximation to the commute time between any two vertices in $O\left(\epsilon^{-2} \log n\right)$ time. This data structure is the natural generalization of a powerful result for undirected graphs: with $O\left(\epsilon^{-2} \log n\right)$ undirected Laplacian solves one can build an $O\left(\epsilon^{-2} \log n\right) \times n$ sized matrix that allows one to compute the effective resistance between any pair of vertices in $O\left(\epsilon^{-2} \log n\right)$ time [54].

This follows from applying the technique used in [54] for sketching effective resistances.

Lemma 50. Let $\mathbf{W} \in \mathcal{R}_{\geq 0}^{n \times n}$ be a random walk matrix corresponding to an irreducible Markov chain with stationary distribution s. Then the commute time $C_{u v}$ satisfies the following three identities:

$$
\begin{aligned}
C_{u v} & =\left(\overrightarrow{1}_{u}-\overrightarrow{1}_{v}\right)^{\top}((\mathbf{I}-\mathbf{W}) \mathbf{S})^{+}\left(\overrightarrow{1}_{u}-\overrightarrow{1}_{v}\right) \\
& =\left(\overrightarrow{1}_{u}-\overrightarrow{1}_{v}\right)^{\top} \mathbf{S}^{-1}(\mathbf{I}-\mathbf{W})^{+}\left(\overrightarrow{1}_{u}-\overrightarrow{1}_{v}\right) \\
& =\left(\overrightarrow{1}_{u}-\overrightarrow{1}_{v}\right)^{\top}\left(\mathcal{L}_{b}^{+}\right)^{\top} \mathbf{U}_{b} \mathcal{L}_{b}^{+}\left(\overrightarrow{1}_{u}-\overrightarrow{1}_{v}\right)
\end{aligned}
$$

where $\mathcal{L}_{b}:=(\mathbf{I}-\mathbf{W}) \mathbf{S}$ is the Laplacian rescaled to an Eulerian graph using the stationary $s$, and $\mathbf{U}_{b}=\frac{1}{2}\left(\mathcal{L}_{b}+\mathcal{L}_{b}^{\top}\right)$. 
We defer the proof to Section C.2 of the Appendix. The connection to effective resistances is more apparent by noticing that for undirected graphs - where $s=\mathbf{D} / 2 m$, and $\mathcal{L}=(\mathbf{I}-\mathbf{W}) \mathbf{D}$ - one obtains $C_{u v}=\left(\overrightarrow{1_{u}}-\overrightarrow{1}_{v}\right)^{\top}\left((\mathbf{I}-\mathbf{W}) \mathbf{D} \cdot \frac{1}{2 m}\right)^{+}\left(\overrightarrow{1}_{u}-\overrightarrow{1}_{v}\right)=2 m \cdot\left(\overrightarrow{1_{u}}-\overrightarrow{1}_{v}\right)^{\top}((\mathbf{I}-\mathbf{W}) \mathbf{D})^{+}\left(\overrightarrow{1}_{u}-\overrightarrow{1}_{v}\right)=2 m \cdot R_{u v}^{\mathrm{eff}}$, which matches a well known identity.

Having shown that $C_{u v}$ can be defined as a quadratic form involving a symmetric positive semidefinite matrix, we can sketch the quadratic form using the technique from [55]. In the remainder of this section we show how to carry out this approach.

One should note that applying the operators $\mathcal{L}_{b}$ and $\mathbf{U}_{b}$ appearing in the symmetric formula requires knowing the stationary distribution. Since our solver provides an approximation $s^{\prime}$ to the stationary, the scaling $\mathcal{L}_{b}^{\prime}=(\mathbf{I}-\mathbf{W}) \mathbf{S}^{\prime}$ will not be Eulerian in general, and therefore its corresponding symmetrization may not even be positive semi-definite. This is addressed by adding extra arcs to $\mathcal{L}_{b}$ so that it becomes Eulerian. If the stationary is computed to high enough accuracy, the additional arcs will not change the commute time by more than a factor of $1+\epsilon / 2$, and the result follows.

For completeness, we first state the Johnson-Lindenstrauss lemma:

Lemma 51. (Johnson-Lindenstrauss) Let $v_{1}, \ldots, v_{m} \in \mathcal{R}^{n}, \epsilon>0$ and $\mathbf{Q}$ a random $k \times n$ matrix where each entry is random $\pm 1 / \sqrt{k}$, with $k \geq 24 \log m / \epsilon^{2}$. Then with high probability, $(1-\epsilon)\left\|v_{i}\right\|_{2}^{2} \leq$ $\left\|\mathbf{Q} v_{i}\right\|_{2}^{2} \leq(1+\epsilon)\left\|v_{i}\right\|_{2}^{2}$, for all $i \in\{1, \ldots, m\}$.

Then we proceed with describing the implementation of the sketch for walk matrices with known stationary distribution. Extending it to the case where the stationary is not known a priori will be done later, by rescaling with an approximate stationary distribution obtained via Theorem 33, and perturbing the problem so that the rescaled matrix becomes exactly balanced.

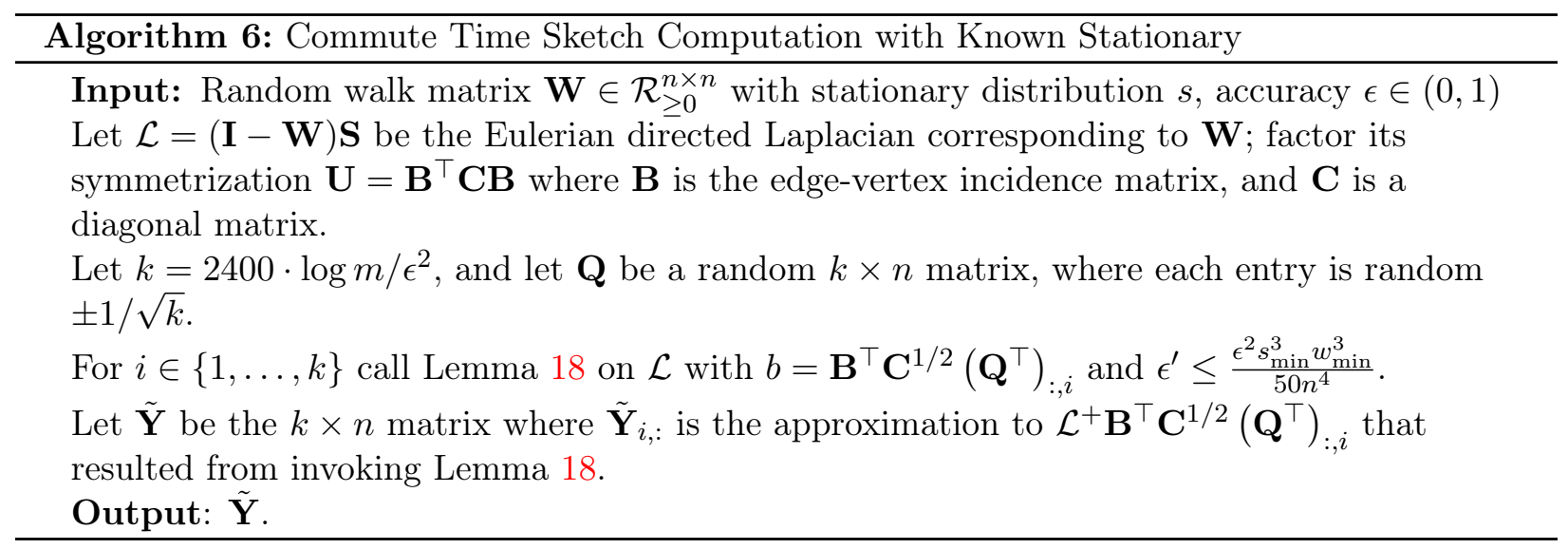

Obtaining an approximation to the commute time $C_{u v}$ is done simply by computing $\| \tilde{\mathbf{Y}}\left(\overrightarrow{1}_{u}-\right.$ $\left.\overrightarrow{1}_{v}\right) \|_{2}^{2}$, as can be seen from the following lemma.

Lemma 52. Let $\mathbf{W} \in \mathcal{R}_{\geq 0}^{n \times n}$ be a random walk matrix with $m$ non-zero entries and a given stationary distribution s, i.e. $(\mathbf{I}-\mathbf{W}) \mathbf{S}$ is an Eulerian directed Laplacian. Let $s_{\min }$ and $w_{\min }$ be the minimum stationary probability and the smallest transition probability, respectively. Then, for any $\epsilon \in(0,1)$, Algorithm 6 computes in time $O\left(\mathcal{T}_{\text {solve }} \cdot \log \left(\frac{n}{\epsilon \cdot s_{\min } w_{\min }}\right) \cdot \frac{1}{\epsilon^{2}}\right)$ a $O\left(\log n / \epsilon^{2}\right) \times n$ matrix $\tilde{\mathbf{Y}}$ such that, $(1-\epsilon) C_{u v} \leq\left\|\tilde{\mathbf{Y}}\left(\overrightarrow{1}_{u}-\overrightarrow{1}_{v}\right)\right\|_{2}^{2} \leq(1+\epsilon) C_{u v}$, with high probability in $n$.

Proof. The proof involves carefully analyzing the composition of errors. Let $\mathbf{X}=\mathcal{L}^{\top} \mathbf{U}^{+} \mathcal{L}, \mathbf{Y}=$ $\mathbf{Q C}^{1 / 2} \mathbf{B}\left(\mathcal{L}^{\top}\right)^{+}$and recall that, by Lemma 18, $\tilde{\mathbf{Y}}$ is an approximation to $\mathbf{Y}$ in the sense that $\left\|\tilde{\mathbf{Y}}_{i,:}-\mathbf{Y}_{i,:}\right\|_{\mathbf{X}} \leq \epsilon^{\prime} \cdot\left\|\mathbf{B}^{\top} \mathbf{C}^{1 / 2}\left(\mathbf{Q}^{\top}\right)_{:, i}\right\|_{\mathbf{U}^{+}}$. 
Using the fact that the commute time $C_{u v}$ can be written as $C_{u v}=\left\|\mathbf{C}^{1 / 2} \mathbf{B}\left(\mathcal{L}^{\top}\right)^{+}\left(\overrightarrow{1}_{u}-\overrightarrow{1}_{v}\right)\right\|^{2}$ (since $\overrightarrow{1}_{u}-\overrightarrow{1}_{v} \perp \overrightarrow{1}$ ), and applying the JL lemma, we have that

$$
(1-\epsilon / 10) C_{u v} \leq\left\|\mathbf{Q} \mathbf{C}^{1 / 2} \mathbf{B}\left(\mathcal{L}^{\top}\right)^{+}\left(\overrightarrow{1}_{u}-\overrightarrow{1}_{v}\right)\right\|^{2}=\left\|\mathbf{Y}\left(\overrightarrow{1}_{u}-\overrightarrow{1}_{v}\right)\right\|_{2}^{2} \leq(1+\epsilon / 10) C_{u v} .
$$

We can bound the error introduced by the Laplacian solver using triangle inequality as follows:

$$
\left|\left\|\tilde{\mathbf{Y}}\left(\overrightarrow{1}_{u}-\overrightarrow{1}_{v}\right)\right\|_{2}-\left\|\mathbf{Y}\left(\overrightarrow{1}_{u}-\overrightarrow{1}_{v}\right)\right\|_{2}\right| \leq\left\|(\tilde{\mathbf{Y}}-\mathbf{Y})\left(\overrightarrow{1}_{u}-\overrightarrow{1}_{v}\right)\right\|_{2}=\sqrt{\sum_{i=1}^{k}\left(\left(\tilde{\mathbf{Y}}_{i,:}-\mathbf{Y}_{i,:}\right)\left(\overrightarrow{1}_{u}-\overrightarrow{1}_{v}\right)\right)^{2}}
$$

Next, let $\Pi=\mathbf{I}-\frac{1}{n} \overrightarrow{1}^{\top}$ be the projection onto the space perpendicular to $\overrightarrow{1}$. Using the fact that both $\overrightarrow{1}_{u}-\overrightarrow{1}_{v}$ and $\left(\tilde{\mathbf{Y}}_{i,:}-\mathbf{Y}_{i,:}\right) \Pi$ are orthogonal to $\operatorname{ker}(\mathbf{X})=\overrightarrow{1}$ (this identity is immediate, since $\mathbf{X}$ corresponds to a doubly stochastic random walk matrix), we can write:

$$
\left(\tilde{\mathbf{Y}}_{i,:}-\mathbf{Y}_{i,:}\right)\left(\overrightarrow{1}_{u}-\overrightarrow{1}_{v}\right)=\left(\tilde{\mathbf{Y}}_{i,:}-\mathbf{Y}_{i,:}\right) \Pi\left(\overrightarrow{1}_{u}-\overrightarrow{1}_{v}\right)=\left(\tilde{\mathbf{Y}}_{i,:}-\mathbf{Y}_{i,:}\right) \Pi \mathbf{X}^{1 / 2} \cdot\left(\mathbf{X}^{+}\right)^{1 / 2} \Pi\left(\overrightarrow{1}_{u}-\overrightarrow{1}_{v}\right),
$$

then apply the Cauchy-Schwarz inequality:

$$
\begin{aligned}
\left(\tilde{\mathbf{Y}}_{i,:}-\mathbf{Y}_{i,:}\right) \Pi \mathbf{X}^{1 / 2} \cdot\left(\mathbf{X}^{+}\right)^{1 / 2} \Pi\left(\overrightarrow{1}_{u}-\overrightarrow{1}_{v}\right) & \leq\left\|\left(\tilde{\mathbf{Y}}_{i,:}-\mathbf{Y}_{i,:}\right) \Pi\right\| \mathbf{X} \cdot\left\|\Pi\left(\overrightarrow{1}_{u}-\overrightarrow{1}_{v}\right)\right\|_{\mathbf{X}^{+}} \\
& =\left\|\tilde{\mathbf{Y}}_{i,:}-\mathbf{Y}_{i,:}\right\| \mathbf{X} \cdot\left\|\overrightarrow{1}_{u}-\overrightarrow{1}_{v}\right\|_{\mathbf{X}^{+}}
\end{aligned}
$$

Combining this with the solver guarantee we obtain

$$
\begin{aligned}
\left|\left\|\tilde{\mathbf{Y}}\left(\overrightarrow{1}_{u}-\overrightarrow{1}_{v}\right)\right\|_{2}-\left\|\mathbf{Y}\left(\overrightarrow{1}_{u}-\overrightarrow{1}_{v}\right)\right\|_{2}\right| & \leq \sqrt{\sum_{i=1}^{k} \epsilon^{\prime} \cdot\left\|\mathbf{B}^{\top} \mathbf{C}^{1 / 2}\left(\mathbf{Q}^{\top}\right)_{:, i}\right\|_{\mathbf{U}^{+}}^{2} \cdot\left\|\overrightarrow{1}_{u}-\overrightarrow{1}_{v}\right\|_{\mathbf{X}^{+}}^{2}} \\
& =\left\|\overrightarrow{1}_{u}-\overrightarrow{1}_{v}\right\|_{\mathbf{X}^{+}} \cdot \sqrt{\epsilon^{\prime}} \cdot \sqrt{\sum_{i=1}^{k}\left\|\mathbf{C}^{1 / 2} \mathbf{B} \mathbf{U}^{+} \mathbf{B}^{\top} \mathbf{C}^{1 / 2}\left(\mathbf{Q}^{\top}\right)_{:, i}\right\|^{2}} \\
& =C_{u v} \cdot \sqrt{\epsilon^{\prime}} \cdot \sqrt{\sum_{i=1}^{k}\left\|\mathbf{C}^{1 / 2} \mathbf{B} \mathbf{U}^{+} \mathbf{B}^{\top} \mathbf{C}^{1 / 2}\left(\mathbf{Q}^{\top}\right)_{:, i}\right\|^{2}} .
\end{aligned}
$$

The factor containing the square root is equal to the Frobenius norm $\left\|\mathbf{C}^{1 / 2} \mathbf{B} \mathbf{U}^{+} \mathbf{B}^{\top} \mathbf{C}^{1 / 2} \mathbf{Q}^{\top}\right\|_{F}$, which can be upper bounded by

$$
\begin{aligned}
\left\|\mathbf{C}^{1 / 2} \mathbf{B} \mathbf{U}^{+} \mathbf{B}^{\top} \mathbf{C}^{1 / 2} \mathbf{Q}^{\top}\right\|_{F} & \leq\left\|\mathbf{C}^{1 / 2} \mathbf{B}\right\|_{F} \cdot\left\|\mathbf{U}^{+} \mathbf{B}^{\top} \mathbf{C}^{1 / 2}\right\|_{F} \cdot\left\|\mathbf{Q}^{\top}\right\|_{F} \\
& =\sqrt{\operatorname{tr}(\mathbf{U}) \cdot \operatorname{tr}\left(\mathbf{U}^{+}\right) \cdot \operatorname{tr}\left(\mathbf{Q}^{\top} \mathbf{Q}\right)} \\
& \leq \sqrt{n \cdot\left(n / \lambda_{\min }(\mathbf{U})\right) \cdot \operatorname{tr}\left(\mathbf{Q}^{\top} \mathbf{Q}\right)} \\
& =\sqrt{n^{3} / \lambda_{\min }(\mathbf{U})}
\end{aligned}
$$

Then, we obtain that

$$
\begin{aligned}
\left|\left\|\tilde{\mathbf{Y}}\left(\overrightarrow{1}_{u}-\overrightarrow{1}_{v}\right)\right\|_{2}-\left\|\mathbf{Y}\left(\overrightarrow{1}_{u}-\overrightarrow{1}_{v}\right)\right\|_{2}\right| & \leq C_{u v} \cdot \sqrt{\epsilon^{\prime}} \cdot \sqrt{n^{3} / \lambda_{\min }(\mathbf{U})} \\
& =C_{u v} \cdot \sqrt{\frac{\epsilon^{2}}{100 n^{3}} \cdot 2 s_{\min }^{2} w_{\min }^{2} \cdot \frac{s_{\min } w_{\min }}{n} \cdot \frac{n^{3}}{\lambda_{\min }(\mathbf{U})}} \leq \sqrt{C_{u v}} \cdot \epsilon / 10 .
\end{aligned}
$$


For the last part we used Cheeger's inequality to bound $\lambda_{\min }(\mathbf{U}) \geq 2\left(s_{\min } w_{\min }\right)^{2}$, and Lemma 55 to bound $C_{u v} \leq n /\left(s_{\min } w_{\min }\right)$.

Therefore

$$
\begin{aligned}
\left|\left\|\tilde{\mathbf{Y}}\left(\overrightarrow{1}_{u}-\overrightarrow{1}_{v}\right)\right\|_{2}^{2}-C_{u v}\right| & \leq\left|\left(\left\|\mathbf{Y}\left(\overrightarrow{1}_{u}-\overrightarrow{1}_{v}\right)\right\|_{2}+\left\|\tilde{\mathbf{Y}}\left(\overrightarrow{1}_{u}-\overrightarrow{1}_{v}\right)\right\|_{2}-\left\|\mathbf{Y}\left(\overrightarrow{1}_{u}-\overrightarrow{1}_{v}\right)\right\|_{2}^{2}-C_{u v}\right)\right| \\
& =\left(\left\|\mathbf{Y}\left(\overrightarrow{1}_{u}-\overrightarrow{1}_{v}\right)\right\|_{2}^{2}-C_{u v}\right)+\left(\left\|\tilde{\mathbf{Y}}\left(\overrightarrow{1}_{u}-\overrightarrow{1}_{v}\right)\right\|_{2}-\left\|\mathbf{Y}\left(\overrightarrow{1}_{u}-\overrightarrow{1}_{v}\right)\right\|_{2}\right)^{2} \\
& +2 \cdot \sqrt{C_{u v}} \cdot\left(\left\|\tilde{\mathbf{Y}}\left(\overrightarrow{1}_{u}-\overrightarrow{1}_{v}\right)\right\|_{2}-\left\|\mathbf{Y}\left(\overrightarrow{1}_{u}-\overrightarrow{1}_{v}\right)\right\|_{2}\right) \\
& \leq C_{u v} \cdot \epsilon / 10+C_{u v} \cdot \epsilon^{2} / 100+2 \cdot C_{u v} \cdot \epsilon / 10 \\
& \leq C_{u v} \cdot \epsilon,
\end{aligned}
$$

and our desired guarantee follows.

The running time is dominated by the $k$ calls to Lemma 18, each of them requiring time $O\left(\mathcal{T}_{\text {solve }} \log \left(1 / \epsilon^{\prime}\right)\right)=O\left(\mathcal{T}_{\text {solve }} \log \frac{n}{\epsilon \cdot s_{\min } w_{\min }}\right)$. Hence the bound on running time.

Next we show how to extend this sketching technique to general walk matrices.

The difficulty lies in the fact that Algorithm 6 requires knowing the exact stationary distribution of $\mathbf{W}$. However, our solver produces only an approximate solution. In order to alleviate this issue, we use the approximate stationary to rescale the Laplacian, then patch the graph by adding extra arcs in order to make it Eulerian. The small error in the stationary approximation guarantees that patching does not significantly change the commute times or the mixing time of the walk matrix. Therefore we can use the patched Laplacian inside Algorithm 6 to produce a sketch. The method is underlined in the algorithm described below.

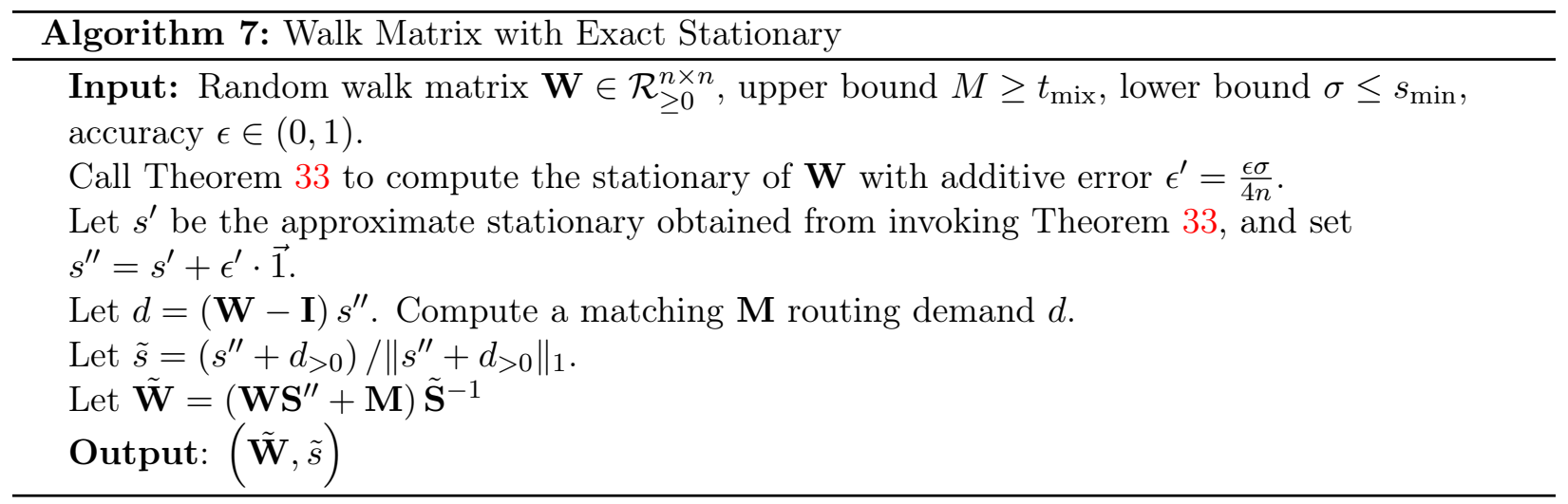

Lemma 53. Let $\mathbf{W} \in \mathcal{R}_{>0}^{n \times n}$ be the random walk matrix of a Markov chain associated with a strongly connected graph. Let $M \geq t_{\operatorname{mix}}$ and $\sigma \leq s_{\min }$. Then Algorithm 7 produces a walk matrix $\tilde{\mathbf{W}}$ such that $\left|\mathbf{W}_{i j}-\tilde{\mathbf{W}}_{i j}\right| \leq \epsilon$ for all $i, j$, along with its exact stationary distribution $\tilde{s}$ in time $O\left(\mathcal{T}_{\text {solve }}\left(\log \frac{n M}{\epsilon \sigma}\right)^{2}\right)$. Furthermore, $s_{i}+\epsilon \sigma / 2 \geq \tilde{s}_{i} \geq s_{i} /(1+\epsilon \sigma)$ for all $i$.

Proof. We start by bounding the $\ell_{1}$ norm of the routed demand $d$. By construction we have $\|d\|_{1}=\left\|(\mathbf{I}-\mathbf{W}) s^{\prime \prime}\right\|_{1}=\left\|(\mathbf{I}-\mathbf{W})\left(s+s^{\prime \prime}-s\right)\right\|_{1}=\left\|(\mathbf{I}-\mathbf{W})\left(s^{\prime \prime}-s\right)\right\|_{1}$. Since applying $\mathbf{W}$ does not increase the $\ell_{1}$ norm of the vector it is applied to, we see that the previous quantity can be bounded by $2\left\|s^{\prime \prime}-s\right\|_{1}=2\left\|\epsilon^{\prime} \overrightarrow{1}+s^{\prime}-s\right\|_{1} \leq 2 n \epsilon^{\prime}+2\left\|s^{\prime}-s\right\|_{1}$. Plugging in the error guarantee $\left\|s^{\prime}-s\right\|_{2} \leq \epsilon^{\prime}$ we obtain $\left\|s^{\prime}-s\right\|_{1} \leq \epsilon^{\prime} \sqrt{n}$. Hence, putting everything together we get $\|d\|_{1} \leq 2 n \epsilon^{\prime}+2 \epsilon^{\prime} \sqrt{n} \leq 4 n \epsilon^{\prime}$. 
Also note that $s_{i}^{\prime \prime} \tilde{s}_{i}^{-1}=\left\|s^{\prime \prime}+d_{>0}\right\|_{1} \cdot \frac{s_{i}^{\prime \prime}}{s_{i}^{\prime \prime}+\left(d_{>0}\right)_{i}}$. Since by construction $s^{\prime \prime} \geq s$, we obtain

$$
\frac{s_{i}^{\prime \prime}}{s_{i}^{\prime \prime}+\|d\|_{1} / 2} \leq s_{i}^{\prime \prime} \tilde{s}_{i}^{-1} \leq\left\|s+\left(s^{\prime \prime}-s\right)+d_{>0}\right\|_{1} .
$$

Using the bound on $\|d\|_{1}$ for the first inequality, plus the triangle inequality for the second, we get

$$
\frac{s_{i}^{\prime \prime}}{s_{i}^{\prime \prime}+2 n \epsilon^{\prime}} \leq s_{i}^{\prime \prime} \tilde{s}_{i}^{-1} \leq 1+4 n \epsilon^{\prime}
$$

for all $i$. Plugging in $\epsilon^{\prime}=\frac{\epsilon \sigma}{4 n}$ we obtain

$$
1-\epsilon \leq s_{i}^{\prime \prime} \tilde{s}_{i}^{-1} \leq 1+\epsilon / 2
$$

Also, note that

$$
\begin{aligned}
\left(\mathbf{M} \tilde{\mathbf{S}}^{-1}\right)_{i j} & \leq\left\|d_{>0}\right\|_{1} \cdot \max _{k}\left(\left\|s^{\prime \prime}+d_{>0}\right\|_{1} \cdot \frac{1}{s_{k}^{\prime \prime}+\left(d_{>0}\right)_{k}}\right) \\
& \leq 2 n \epsilon^{\prime} \cdot\left(\left(1+4 n \epsilon^{\prime}\right) \cdot \frac{1}{s_{\min }}\right) \leq \epsilon / 2 .
\end{aligned}
$$

Combining Equations 7.5 and 7.6, we obtain that $(1-\epsilon) \mathbf{W}_{i j} \leq \tilde{\mathbf{W}}_{i j} \leq(1+\epsilon / 2) \mathbf{W}_{i j}+\epsilon / 2$, and the desired bound on $\tilde{\mathbf{W}}_{i j}$ follows.

Also, by construction, we notice that $\tilde{s}_{i} \geq s_{i}^{\prime \prime} /\left\|s^{\prime \prime}+d_{>0}\right\|_{1} \geq s_{i} /\left(1+4 n \epsilon^{\prime}\right)=s_{i} /(1+\epsilon \sigma)$. Similarly, since $s^{\prime \prime} \geq s$, and therefore $\tilde{s}_{i} \leq s_{i}+\left\|d_{>0}\right\|_{1}$, we have $\tilde{s}_{i} \leq s_{i}+2 n \epsilon^{\prime} \leq s_{i}+\epsilon \sigma / 2$.

The running time is dominated by the invocation of Theorem 33, which requires time $O\left(\mathcal{T}_{\text {solve }}\left(\log \frac{n M}{\epsilon^{\prime}}\right)^{2}\right)=O\left(\mathcal{T}_{\text {solve }}\left(\log \frac{n M}{\epsilon \sigma}\right)^{2}\right)$.

The lemma below shows a sufficient accuracy to which we need to invoke Algorithm 7 in order to approximately preserve commute times.

Lemma 54. Let $\mathbf{W}, \tilde{\mathbf{W}} \in \mathcal{R}_{\geq 0}^{n \times n}$ be random walk matrices of Markov chains associated with strongly connected graphs, having stationaries $s$ and $\tilde{s}$, respectively. Let $C$ and $\tilde{C}$, respectively, denote commute times for the two walk matrices. Let $s_{\min }$ represent the minimum stationary probability of $\mathbf{W}$, and let $\epsilon_{0} \leq \epsilon \cdot s_{\min }^{2} /\left(256 \cdot n^{3} \log ^{2} n \cdot t_{\text {mix }}^{2}\right)$. If $\left|\mathbf{W}_{i j}-\tilde{\mathbf{W}}_{i j}\right| \leq \epsilon_{0}$ for all $i, j$, and $\|s-\tilde{s}\|_{\infty} \leq \epsilon_{0}$, then $\left|\tilde{C}_{u v}-C_{u v}\right| \leq \epsilon$, for all $u, v$.

Proof. Recall that, by Lemma 50, we can write $C_{u v}=\left(\overrightarrow{1}_{u}-\overrightarrow{1}_{v}\right)^{\top} \mathbf{S}^{-1}(\mathbf{I}-\mathbf{W})^{+}\left(\overrightarrow{1}_{u}-\overrightarrow{1}_{v}\right)$, and similarly for $\tilde{C}_{u v}$. We proceed to bounding the difference between them as follows:

$$
\begin{aligned}
\left|C_{u v}-\tilde{C}_{u v}\right| & =\left|\left(\overrightarrow{1}_{u}-\overrightarrow{1}_{v}\right)^{\top}\left(((\mathbf{I}-\mathbf{W}) \mathbf{S})^{+}-((\mathbf{I}-\tilde{\mathbf{W}}) \tilde{\mathbf{S}})^{+}\right)\left(\overrightarrow{1}_{u}-\overrightarrow{1}_{v}\right)\right| \\
& \left.\leq\left\|\overrightarrow{1}_{u}-\overrightarrow{1}_{v}\right\| \|((\mathbf{I}-\mathbf{W}) \mathbf{S})^{+}-((\mathbf{I}-\tilde{\mathbf{W}}) \tilde{\mathbf{S}})^{+}\right)\left(\overrightarrow{1}_{u}-\overrightarrow{1}_{v}\right) \| .
\end{aligned}
$$

Our goal will be to apply Lemma 66 with $\mathbf{A}=(\mathbf{I}-\mathbf{W}) \mathbf{S}, \Delta \mathbf{A}=(\mathbf{I}-\tilde{\mathbf{W}}) \tilde{\mathbf{S}}-(\mathbf{I}-\mathbf{W}) \mathbf{S}$, $b=e_{u}-e_{v}$. First let us bound $\left\|\mathbf{A}^{+}\right\|$and $\|\Delta \mathbf{A}\|$. 
First, we bound

$$
\begin{aligned}
\left\|\mathbf{A}^{+}\right\| & =\left\|((\mathbf{I}-\mathbf{W}) \mathbf{S})^{+}\right\|=\max _{x:(\mathbf{I}-\mathbf{W}) \mathbf{S} x \neq 0} \frac{\|x\|}{\|(\mathbf{I}-\mathbf{W}) \mathbf{S} x\|} \\
& \leq \max _{x:(\mathbf{I}-\mathbf{W}) \mathbf{S} x \neq 0} \frac{\left\|\mathbf{S}^{-1}\right\|\|\mathbf{S} x\|}{\|(\mathbf{I}-\mathbf{W}) \mathbf{S} x\|}=\left\|\mathbf{S}^{-1}\right\|\left\|(\mathbf{I}-\mathbf{W})^{+}\right\| \\
& \leq 4 \cdot n \log n \cdot t_{\text {mix }} / s_{\text {min }} .
\end{aligned}
$$

For the last inequality, we used the bound on $\left\|(\mathbf{I}-\mathbf{W})^{+}\right\|$from Theorem 21 .

Second, we bound $\|\Delta \mathbf{A}\|$. Applying triangle inequality, we obtain

$$
\begin{aligned}
\|\Delta \mathbf{A}\| & \leq\|((\mathbf{I}-\tilde{\mathbf{W}})-(\mathbf{I}-\mathbf{W})) \tilde{\mathbf{S}}\|+\|(\mathbf{I}-\mathbf{W})(\tilde{\mathbf{S}}-\mathbf{S})\| \\
& \leq\|\tilde{\mathbf{W}}-\mathbf{W}\|\|\tilde{\mathbf{S}}\|+\|\mathbf{I}-\mathbf{W}\|\|\tilde{\mathbf{S}}-\mathbf{S}\| .
\end{aligned}
$$

Since we have an entry-wise bound on the difference between $\tilde{\mathbf{W}}$ and $\mathbf{W}$, we can upper bound $\|\tilde{\mathbf{W}}-\mathbf{W}\|$ with the Frobenius norm $\|\tilde{\mathbf{W}}-\mathbf{W}\|_{F} \leq n \epsilon_{0}$. Also $\tilde{\mathbf{S}}$ is a stationary, so $\|\tilde{\mathbf{S}}\| \leq 1$. Additionally, the additive error on $\tilde{s}$, bounds $\|\tilde{\mathbf{S}}-\mathbf{S}\| \leq \epsilon_{0}$. Also $\|\mathbf{I}-\mathbf{W}\|_{2} \leq \sqrt{\|\mathbf{I}-\mathbf{W}\|_{1} \cdot\|\mathbf{I}-\mathbf{W}\|_{\infty}} \leq$ $\sqrt{2 \cdot n}$. Combining these, we obtain

$$
\|\Delta \mathbf{A}\| \leq n \epsilon_{0}+\sqrt{2 n} \cdot \epsilon_{0} \leq \frac{1}{2\left\|\mathbf{A}^{+}\right\|}
$$

Therefore we can plug our bounds into in our bounds into Lemma 66, and obtain $\frac{\left\|\left(\mathbf{A}^{++}-\mathbf{A}^{+}\right) b\right\|}{\left\|\mathbf{A}^{+} b\right\|} \leq$ $\frac{\left\|\mathbf{A}^{+}\right\|\|\Delta \mathbf{A}\|}{1-\left\|\mathbf{A}^{+}\right\|\|\Delta \mathbf{A}\|} \leq 2\left\|\mathbf{A}^{+}\right\|\|\Delta \mathbf{A}\|$, and equivalently $\left\|\left(\mathbf{A}^{\prime+}-\mathbf{A}^{+}\right) b\right\| \leq 2\left\|\mathbf{A}^{+}\right\|^{2}\|\Delta \mathbf{A}\|\|b\|$.

Finally, combining with Equation 7.8 , we obtain $\left|\tilde{C}_{u v}-C_{u v}\right| \leq \sqrt{2} \cdot 2\left\|\mathbf{A}^{+}\right\|^{2}\|\Delta \mathbf{A}\|\|b\| \leq 4$. $\left(\frac{4 n \log n \cdot t_{\text {mix }}}{s_{\min }}\right)^{2} \cdot\left(n \epsilon_{0}+\sqrt{2 n} \cdot \epsilon_{0}\right) \leq \epsilon$

Lemma 55. (Bounding commute times) Let $\mathbf{W} \in \mathcal{R}_{\geq 0}^{n \times n}$ be a random walk matrix corresponding to an irreducible Markov chain, and let $s_{\min }$ and $w_{\min }$ be its smallest stationary probability, and smallest transition probability, respectively. Then any commute time $C_{u v}$ satisfies $C_{u v} \leq n /\left(s_{\min } w_{\min }\right)$.

Proof. We will use the fact that commute times form a metric. Then any commute time $C_{u v}$ is upper bounded by the sum of commute times over arcs in $\mathbf{W}$ with nonzero probability. Using Lemma 50, we can therefore bound

$$
\begin{aligned}
C_{u v} & \leq \sum_{i \neq j: \mathbf{W}_{i j}>0}\left(\overrightarrow{1}_{i}-\overrightarrow{1}_{j}\right)^{\top}\left(\mathcal{L}_{b} \mathbf{U}_{b}^{+} \mathcal{L}_{b}^{\top}\right)^{+}\left(\overrightarrow{1}_{i}-\overrightarrow{1}_{j}\right) \\
& \leq \frac{1}{\min _{i \neq j: \mathbf{W}_{i j}>0}\left|\mathbf{U}_{b}\right|_{i j}} \cdot \sum_{i \neq j: \mathbf{W}_{i j}>0}\left(\mathcal{L}_{b} \mathbf{U}_{b}^{+} \mathcal{L}_{b}^{\top}\right)^{+} \bullet\left(\left(\overrightarrow{1}_{i}-\overrightarrow{1}_{j}\right)\left(\overrightarrow{1}_{i}-\overrightarrow{1}_{j}\right)^{\top} \cdot\left|\mathbf{U}_{b}\right|_{i j}\right) \\
& \leq \frac{1}{\min _{i \neq j: \mathbf{W}_{i j}>0}\left|\mathbf{U}_{b}\right|_{i j}} \cdot \operatorname{tr}\left(\left(\mathcal{L}_{b} \mathbf{U}_{b}^{+} \mathcal{L}_{b}^{\top}\right)^{+} \mathbf{U}_{b}\right) .
\end{aligned}
$$

From Lemma 13 we have that $\mathcal{L}_{b} \mathbf{U}_{b}^{+} \mathcal{L}_{b}^{\top} \succeq \mathbf{U}_{b}$, and therefore $\left(\mathcal{L}_{b} \mathbf{U}_{b}^{+} \mathcal{L}_{b}^{\top}\right)^{+} \preceq \mathbf{U}_{b}^{+}$. Hence $\operatorname{tr}\left(\left(\mathcal{L}_{b} \mathbf{U}_{b}^{+} \mathcal{L}_{b}^{\top}\right)^{+} \mathbf{U}_{b}\right) \leq \operatorname{tr}\left(\mathbf{U}_{b}^{+} \mathbf{U}_{b}\right)=n-1$. So

$$
C_{u v} \leq \frac{n-1}{\min _{i \neq j: \mathbf{W}_{i j}>0}\left|\mathbf{U}_{b}\right|_{i j}} .
$$


Finally, since $\left(\mathbf{U}_{b}\right)_{i j} \geq\left(\mathcal{L}_{b}\right)_{i j}=\left(\left(\mathbf{I}-\mathbf{W}^{\top}\right) \mathbf{S}\right)_{i j}$, we have that

$$
\min _{i \neq j: \mathbf{W}_{i j}>0}\left|\mathbf{U}_{b}\right|_{i j} \geq \min _{i \neq j: \mathbf{W}_{i j}>0}\left(\mathbf{W}^{\top} \mathbf{S}\right)_{i j} \geq s_{\min } w_{\min }
$$

Therefore

$$
C_{u v} \leq \frac{n-1}{s_{\min } w_{\min }}
$$

Finally, we can combine Lemmas 52, 53, and 54 in order to obtain the following result:

Theorem 56. Let $\mathbf{W} \in \mathcal{R}_{\geq 0}^{n \times n}$ be the random walk matrix of a Markov chain associated with a strongly connected graph. Let $M \geq t_{\operatorname{mix}}, \sigma \leq s_{\min }$, where $s_{\min }$ represents the smallest stationary probability, and let $w_{\min }$ be the smallest transition probability. Then Algorithm 8 computes in time $O\left(\mathcal{T}_{\text {solve }}\left(\left(\log \frac{n M}{\epsilon \sigma}\right)^{2}+\frac{1}{\epsilon^{2}} \log \frac{n}{\epsilon \sigma w_{\min }}\right)\right)$ an $O\left(\log n / \epsilon^{2}\right) \times n$ matrix $\tilde{\mathbf{Y}}$ such that, $(1-\epsilon) C_{u v} \leq$ $\left\|\tilde{\mathbf{Y}}\left(e_{u}-e_{v}\right)\right\|_{2}^{2} \leq(1+\epsilon) C_{u v}$, with high probability in $n$.

Proof. We start by proving correctness. First, we see that the commute times in $\tilde{\mathbf{W}}$ approximate the original ones within $1+\epsilon / 2$. Indeed, as seen in Lemma 53, running Algorithm 7 on $\mathbf{W}$ with accuracy $\epsilon_{0}=\frac{\sigma^{2}}{256 \cdot n^{3} \log ^{2} n \cdot M^{2}} \cdot \frac{\epsilon}{2}$ produces a walk matrix $\tilde{\mathbf{W}}$ with stationary $\tilde{s}$, whose entries approximate those of $\mathbf{W}$ within additive error $\epsilon_{0}$. Furthermore $s_{i}+\epsilon_{0} \sigma / 2 \geq \tilde{s}_{i} \geq s_{i} /\left(1+\epsilon_{0} \sigma\right)$, which immediately implies $\|\tilde{s}-s\|_{\infty} \leq \epsilon_{0}$. Hence, by Lemma 54, $\left|\tilde{C}_{u v}-C_{u v}\right| \leq \epsilon / 2$ for all $u, v$. Then, running Algorithm 6 on $(\tilde{\mathbf{W}}, \tilde{s})$ we obtain a matrix $\tilde{\mathbf{Y}}$, which we can use to sketch all commute times in $\tilde{\mathbf{W}}$ with multiplicative error $1+\epsilon / 3$. Therefore $\tilde{\mathbf{Y}}$ sketches commute times in $\mathbf{W}$ with multiplicative error $1+\epsilon$.

Next, we bound the total running time. The call to Algorithm 7 requires time $O\left(\mathcal{T}_{\text {solve }}\left(\log \frac{n M}{\epsilon_{0} \sigma}\right)^{2}\right)=$ $O\left(\mathcal{T}_{\text {solve }}\left(\log \frac{n M}{\epsilon \sigma}\right)^{2}\right)$. The running time of the call to Algorithm 6 depends on the minimum stationary probability, and the smallest transition probability of $\tilde{\mathbf{W}}: \tilde{s}_{\min }$ and $\tilde{w}_{\text {min }}$, respectively. From Lemma 53, we see that these are within constant factors from the originals. Therefore, the running time of this call is $O\left(\mathcal{T}_{\text {solve }} \cdot \log \frac{n}{\epsilon \sigma w_{\min }} \cdot \frac{1}{\epsilon^{2}}\right)$. Putting these bounds together, we obtain our bound.

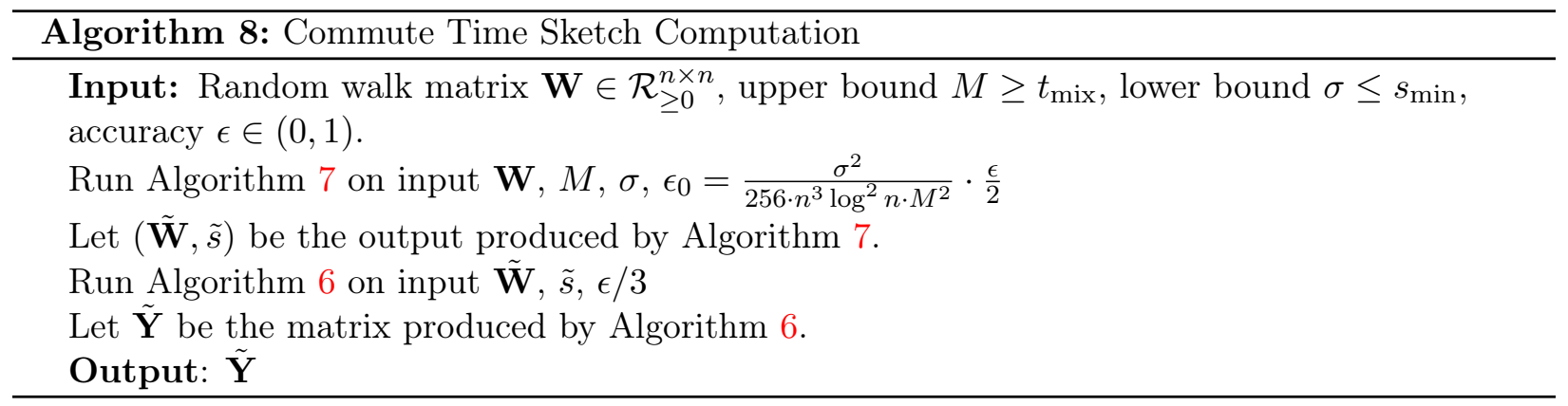

\section{References}

[1] Zeyuan Allen-Zhu, Zhenyu Liao, and Lorenzo Orecchia. Spectral sparsification and regret minimization beyond matrix multiplicative updates. In Proceedings of the Forty-Seventh Annual ACM on Symposium on Theory of Computing, pages 237-245. ACM, 2015. 
[2] Noga Alon and Vitali D Milman. $\lambda 1$, isoperimetric inequalities for graphs, and superconcentrators. Journal of Combinatorial Theory, Series B, 38(1):73-88, 1985.

[3] Nima Anari and Shayan Oveis Gharan. Effective-resistance-reducing flows, spectrally thin trees, and asymmetric tsp. In Foundations of Computer Science (FOCS), 2015 IEEE 56th Annual Symposium on, pages 20-39. IEEE, 2015.

[4] Reid Andersen, Christian Borgs, Jennifer Chayes, John Hopcraft, Vahab S Mirrokni, and Shang-Hua Teng. Local computation of pagerank contributions. In International Workshop on Algorithms and Models for the Web-Graph, pages 150-165. Springer, 2007.

[5] Reid Andersen, Fan Chung, and Kevin Lang. Local graph partitioning using pagerank vectors. In Proceedings of the 47th Annual IEEE Symposium on Foundations of Computer Science, FOCS '06, pages 475-486, Washington, DC, USA, 2006. IEEE Computer Society.

[6] Arash Asadpour, Michel X Goemans, Aleksander Madry, Shayan Oveis Gharan, and Amin Saberi. An o $(\log \mathrm{n} / \log \log \mathrm{n})$-approximation algorithm for the asymmetric traveling salesman problem. In SODA, volume 10, pages 379-389. SIAM, 2010.

[7] Paul Christiano, Jonathan A Kelner, Aleksander Madry, Daniel A Spielman, and Shang-Hua Teng. Electrical flows, laplacian systems, and faster approximation of maximum flow in undirected graphs. In Proceedings of the forty-third annual ACM symposium on Theory of computing, pages 273-282. ACM, 2011.

[8] Fan Chung. Laplacians and the cheeger inequality for directed graphs. Annals of Combinatorics, 9(1):1-19, 2005.

[9] K. M. Chung, O. Reingold, and S. Vadhan. S-t connectivity on digraphs with a known stationary distribution. In Computational Complexity, 200\%. CCC '0\%. Twenty-Second Annual IEEE Conference on, pages 236-249, June 2007.

[10] Michael B. Cohen, Rasmus Kyng, Gary L. Miller, Jakub W. Pachocki, Richard Peng, Anup B. $\mathrm{Rao}$, and Shen Chen $\mathrm{Xu}$. Solving sdd linear systems in nearly mlog $1 / 2 \mathrm{n}$ time. In Proceedings of the 46th Annual ACM Symposium on Theory of Computing, STOC '14, pages 343-352, New York, NY, USA, 2014. ACM.

[11] Michael B. Cohen, Aleksander Madry, Piotr Sankowski, and Adrian Vladu. Negative-weight shortest paths and unit capacity minimum cost flow in $\tilde{\mathrm{o}}\left(\mathrm{m}^{10 / 7} \log \mathrm{W}\right)$ time. CoRR, abs/1605.01717, 2016.

[12] Samuel I. Daitch and Daniel A. Spielman. Faster approximate lossy generalized flow via interior point algorithms. In Proceedings of the Fortieth Annual ACM Symposium on Theory of Computing, STOC '08, pages 451-460, New York, NY, USA, 2008. ACM.

[13] Martin Dyer, Alan Frieze, and Ravi Kannan. A random polynomial-time algorithm for approximating the volume of convex bodies. Journal of the ACM (JACM), 38(1):1-17, 1991.

[14] Dániel Fogaras and Balázs Rácz. Towards scaling fully personalized pagerank. In International Workshop on Algorithms and Models for the Web-Graph, pages 105-117. Springer, 2004.

[15] K. Guo and B. Mohar. Hermitian adjacency matrix of digraphs and mixed graphs. ArXiv e-prints, May 2015. 
[16] Nicholas J Higham. Accuracy and stability of numerical algorithms. Siam, 2002.

[17] Shlomo Hoory, Nathan Linial, and Avi Wigderson. Expander graphs and their applications. Bulletin of the American Mathematical Society, 43(4):439-561, 2006.

[18] Glen Jeh and Jennifer Widom. Scaling personalized web search. In Proceedings of the 12th international conference on World Wide Web, pages 271-279. ACM, 2003.

[19] Mark Jerrum, Alistair Sinclair, and Eric Vigoda. A polynomial-time approximation algorithm for the permanent of a matrix with nonnegative entries. Journal of the ACM (JACM), 51(4):671-697, 2004.

[20] Ravi Kannan, Santosh Vempala, and Adrian Vetta. On clusterings: Good, bad and spectral. Journal of the ACM (JACM), 51(3):497-515, 2004.

[21] Richard M Karp, Michael Luby, and Neal Madras. Monte-carlo approximation algorithms for enumeration problems. Journal of algorithms, 10(3):429-448, 1989.

[22] Jonathan A Kelner, Yin Tat Lee, Lorenzo Orecchia, and Aaron Sidford. An almost-linear-time algorithm for approximate max flow in undirected graphs, and its multicommodity generalizations. In Proceedings of the Twenty-Fifth Annual ACM-SIAM Symposium on Discrete Algorithms, pages 217-226. Society for Industrial and Applied Mathematics, 2014.

[23] Jonathan A Kelner and Aleksander Madry. Faster generation of random spanning trees. In Foundations of Computer Science, 2009. FOCS'09. 50th Annual IEEE Symposium on, pages 13-21. IEEE, 2009.

[24] Jonathan A Kelner, Gary L Miller, and Richard Peng. Faster approximate multicommodity flow using quadratically coupled flows. In Proceedings of the forty-fourth annual ACM symposium on Theory of computing, pages 1-18. ACM, 2012.

[25] Jonathan A. Kelner, Lorenzo Orecchia, Aaron Sidford, and Zeyuan Allen Zhu. A simple, combinatorial algorithm for solving SDD systems in nearly-linear time. In Proceedings of the 45th annual ACM symposium on Symposium on theory of computing, STOC '13, pages 911-920, New York, NY, USA, 2013. ACM. Available at http://arxiv.org/abs/1301.6628.

[26] I. Koutis, G. Miller, and R. Peng. Approaching optimality for solving sdd linear systems. SIAM Journal on Computing, 43(1):337-354, 2014.

[27] Ioannis Koutis, Gary L. Miller, and Richard Peng. Approaching optimality for solving SDD linear systems. In Proceedings of the 2010 IEEE 51st Annual Symposium on Foundations of Computer Science, FOCS '10, pages 235-244, Washington, DC, USA, 2010. IEEE Computer Society. Available at http://arxiv.org/abs/1003.2958.

[28] Ioannis Koutis, Gary L. Miller, and Richard Peng. A nearly-m log n time solver for SDD linear systems. In Proceedings of the 2011 IEEE 52nd Annual Symposium on Foundations of Computer Science, FOCS '11, pages 590-598, Washington, DC, USA, 2011. IEEE Computer Society. Available at http://arxiv.org/abs/1102.4842.

[29] Rasmus Kyng, Yin Tat Lee, Richard Peng, Sushant Sachdeva, and Daniel A. Spielman. Sparsified Cholesky and multigrid solvers for connection Laplacians. CoRR, abs/1512.01892, 2015. Available at http://arxiv.org/abs/1512.01892. 
[30] Y. T. Lee and H. Sun. Constructing linear-sized spectral sparsification in almost-linear time. In Foundations of Computer Science (FOCS), 2015 IEEE 56th Annual Symposium on, pages 250-269, Oct 2015.

[31] Yin Tat Lee, Richard Peng, and Daniel A. Spielman. Sparsified cholesky solvers for SDD linear systems. CoRR, abs/1506.08204, 2015.

[32] Yin Tat Lee, Satish Rao, and Nikhil Srivastava. A new approach to computing maximum flows using electrical flows. In Proceedings of the forty-fifth annual ACM symposium on Theory of computing, pages 755-764. ACM, 2013.

[33] Yin Tat Lee and Aaron Sidford. Efficient accelerated coordinate descent methods and faster algorithms for solving linear systems. In Foundations of Computer Science (FOCS), 2013 IEEE 54th Annual Symposium on, pages 147-156. IEEE, 2013.

[34] Yin Tat Lee and Aaron Sidford. Path finding methods for linear programming: Solving linear programs in $\tilde{O} \sqrt{\text { rank }}$ iterations and faster algorithms for maximum flow. In Foundations of Computer Science (FOCS), 2014 IEEE 55th Annual Symposium on, pages 424-433. IEEE, 2014. Available at http://arxiv.org/abs/1312.6677 and http://arxiv.org/abs/1312.6713.

[35] Yin Tat Lee and Santosh S Vempala. Geodesic walks on polytopes. arXiv preprint arXiv:1606.04696, 2016.

[36] Peter A Lofgren, Siddhartha Banerjee, Ashish Goel, and C Seshadhri. Fast-ppr: scaling personalized pagerank estimation for large graphs. In Proceedings of the 20th ACM SIGKDD international conference on Knowledge discovery and data mining, pages 1436-1445. ACM, 2014.

[37] László Lovász. Random walks on graphs. Combinatorics, Paul erdos is eighty, 2:1-46, 1993.

[38] László Lovász and Miklós Simonovits. The mixing rate of markov chains, an isoperimetric inequality, and computing the volume. In Foundations of Computer Science, 1990. Proceedings., 31st Annual Symposium on, pages 346-354. IEEE, 1990.

[39] László Lovász and Santosh Vempala. Fast algorithms for logconcave functions: Sampling, rounding, integration and optimization. In 2006 47th Annual IEEE Symposium on Foundations of Computer Science (FOCS'06), pages 57-68. IEEE, 2006.

[40] László Lovász and Santosh Vempala. Hit-and-run from a corner. SIAM Journal on Computing, 35(4):985-1005, 2006.

[41] Aleksander Madry. Navigating central path with electrical flows: From flows to matchings, and back. In 54th Annual IEEE Symposium on Foundations of Computer Science, FOCS 2013, 26-29 October, 2013, Berkeley, CA, USA, pages 253-262, 2013.

[42] Aleksander Mądry, Damian Straszak, and Jakub Tarnawski. Fast generation of random spanning trees and the effective resistance metric. In Proceedings of the Twenty-Sixth Annual ACM-SIAM Symposium on Discrete Algorithms, pages 2019-2036. SIAM, 2015.

[43] Lorenzo Orecchia, Sushant Sachdeva, and Nisheeth K Vishnoi. Approximating the exponential, the lanczos method and an o (m)-time spectral algorithm for balanced separator. In Proceedings of the forty-fourth annual ACM symposium on Theory of computing, pages 1141-1160. ACM, 2012. 
[44] Lawrence Page, Sergey Brin, Rajeev Motwani, and Terry Winograd. The pagerank citation ranking: bringing order to the web. Technical report, Stanford University, 1999.

[45] Richard Peng. Algorithm Design Using Spectral Graph Theory. PhD thesis, Carnegie Mellon University, Pittsburgh, August 2013. CMU CS Tech Report CMU-CS-13-121, available at http://reports-archive.adm.cs.cmu.edu/anon/2013/CMU-CS-13-121.pdf.

[46] Richard Peng. Approximate undirected maximum flows in $O(m$ polylog $(n))$ time. In Proceedings of the Twenty-Seventh Annual ACM-SIAM Symposium on Discrete Algorithms, SODA 2016, Arlington, VA, USA, January 10-12, 2016, pages 1862-1867, 2016.

[47] Richard Peng and Daniel A. Spielman. An efficient parallel solver for SDD linear systems. In Proceedings of the 46th Annual ACM Symposium on Theory of Computing, STOC '14, pages 333-342, New York, NY, USA, 2014. ACM. Available at http://arxiv.org/abs/1311.3286.

[48] Omer Reingold. Undirected connectivity in log-space. Journal of the ACM (JACM), 55(4):17, 2008.

[49] Jonah Sherman. Nearly maximum flows in nearly linear time. In Foundations of Computer Science (FOCS), 2013 IEEE 54th Annual Symposium on, pages 263-269. IEEE, 2013.

[50] Alistair Sinclair and Mark Jerrum. Approximate counting, uniform generation and rapidly mixing markov chains. Inf. Comput., 82(1):93-133, 1989.

[51] Michael Sipser and Daniel A Spielman. Expander codes. IEEE Transactions on Information Theory, 42(6):1710-1722, 1996.

[52] D. Spielman and S. Teng. Nearly linear time algorithms for preconditioning and solving symmetric, diagonally dominant linear systems. SIAM Journal on Matrix Analysis and Applications, 35(3):835-885, 2014. Available at http://arxiv.org/abs/cs/0607105.

[53] Daniel A Spielman. Linear-time encodable and decodable error-correcting codes. In Proceedings of the twenty-seventh annual ACM symposium on Theory of computing, pages 388-397. ACM, 1995.

[54] Daniel A. Spielman and Nikhil Srivastava. Graph sparsification by effective resistances. In Proceedings of the Fortieth Annual ACM Symposium on Theory of Computing, STOC '08, pages 563-568, New York, NY, USA, 2008. ACM.

[55] Daniel A. Spielman and Nikhil Srivastava. Graph sparsification by effective resistances. SIAM Journal on Computing, 40(6):1913-1926, 2011. Announced at STOC'08.

[56] Daniel A. Spielman and Jaeoh Woo. A note on preconditioning by low-stretch spanning trees. CoRR, abs/0903.2816, 2009.

[57] Shang-Hua Teng. The laplacian paradigm: Emerging algorithms for massive graphs. In Proceedings of the 7th Annual Conference on Theory and Applications of Models of Computation, TAMC'10, pages 2-14, Berlin, Heidelberg, 2010. Springer-Verlag.

[58] Joel A Tropp. User-friendly tail bounds for sums of random matrices. Foundations of computational mathematics, 12(4):389-434, 2012.

[59] Santosh Vempala. Geometric random walks: a survey. Combinatorial and computational geometry, 52(573-612):2, 2005. 
[60] Virginia Vassilevska Williams. Multiplying matrices faster than coppersmith-winograd. In Proceedings of the 44th Symposium on Theory of Computing Conference, STOC 2012, New York, NY, USA, May 19 - 22, 2012, pages 887-898, 2012.

\section{A Numerical Stability}

In this section we give brief justifications of the numerical stability of our routine. For simplicity we will use fixed point arithmetic, and show that $O(1)$ words are sufficient when the input fits in word size. However, we do assume the numerical errors are adversarial: specifically we assume that at each step, any value can be perturbed adversarially by machine epsilon $\left(\epsilon_{\operatorname{mac}}\right)$.

Formally our assumptions include:

1. The numerical stability of Gaussian elimination / LU factorization. Specifically given a matrix $M$ with condition number $\kappa$, we can access its inverse $M^{-1}$ with additive error $\epsilon$ in $O\left(n^{3} \log ^{O(1)}\left(\kappa \epsilon^{-1}\right)\right)$ time by manipulating words of length $O\left(\log \left(\kappa \epsilon^{-1}\right)\right)$ : see i.e. [16], Chapters 9 and 10, in particular Theorems 9.5. and 10.8 .

2. Numerically stable solvers for undirected Laplacians: given a graph Laplacian where all weights are in the range $[1, \mathrm{U}]$, solves in it can be made to $\epsilon$ additive error in time $O\left(m \log O(1)(n) \log \left(U \epsilon^{-1}\right)\right)$ by manipulating words of length $O\left(\log \left(U \epsilon^{-1}\right)\right)$.

3. The stability of iterative refinement: once we obtain a constant factor approximation, we can obtain an $\epsilon$-approximation in a numerically stable manner by working with words of the same length.

Our analyses center around bounding the condition numbers of the matrices involved. This is because our algorithm makes crucial use of the Woodbury formula, which preserves condition number bounds, but may severely change the matrix entry-wise. Recall that $\kappa(\cdot)$ denotes the condition number of a matrix, which in case of symmetric matrices/operators is the maximum over minimum eigenvalue.

\section{A.1 Reduction to Eulerian Case}

Here it suffices to check that the iterative process outlined in Theorem 2 can tolerate perturbations to its intermediate vectors. Its proof relies on showing the 1-norm of a vector, $\left\|\mathbf{D}^{-1}\left(e^{(t-1)}-\alpha d\right)\right\|_{1}$ is geometrically decreasing until up to $32 \cdot \alpha \cdot|V|$. Such a bound is friendly to perturbations by vectors that are entrywise less than $\alpha$. Furthermore, if the entries of $D$ are at least $U^{-1}$, perturbations less than $U^{-1} \alpha$ are sufficient. The most important condition is that the intermediate matrices generated by this reduction do not become ill-conditioned.

Lemma 57. If Algorithm 1 takes a directed Laplacian $\mathcal{L} \in \mathcal{R}^{n \times n}$ where all non-zero entries are within a factor of $\kappa$ of each other, and restart parameter $\alpha$, then all intermediate matrices $\left(\mathbf{M}^{(t)}\right)$ that it performs linear-system solve on satisfy $\kappa\left(\frac{1}{2}\left(\mathbf{M}+\mathbf{M}^{\top}\right)\right) \leq O\left(\kappa^{2} \alpha^{-2} n^{5}\right)$.

Proof. The proof of Theorem 2 in Section 3.3.5 gives that all entries in $\mathbf{D} \mathbf{X}^{(t)}$ are within a ratio of $20 \alpha^{-2} n$ of each other. As $\mathbf{D}$ is the diagonal of $\mathcal{L}$, all entries in $\mathbf{D}$ are within a factor of $\kappa n$ of each other, and therefore all entries of $\mathbf{X}^{(t)}$ are within a factor of $20 \kappa \alpha^{-2} n$ of each other.

Therefore all non-zero entries in the matrix $\mathcal{L} \mathbf{X}^{(t)}$ are within a factor of $20 \kappa^{2} \alpha^{-2} n$ of each other. Since the matrix $\mathbf{E}^{(t)}$ is chosen so that $\mathbf{E}^{(t)} \mathbf{X}^{(t)}+\mathcal{L} \mathbf{X}^{(t)}$ is $\alpha$-RCDD and $\alpha \leq \frac{1}{2}$, the additional entries can equal to at most the row/column sums of $\mathcal{L} \mathbf{X}^{(t)}$. Therefore all non-zero entries in $\mathbf{M}$ are 
within a factor of $20 \kappa^{2} \alpha^{-2} n^{2}$ of each other, and the choice of $\mathbf{U}=\frac{1}{2}\left(\mathbf{M}+\mathbf{M}^{\top}\right)$ means all non-zero entries of the SDD matrix $\mathbf{U}$ are within a factor of $40 \kappa^{2} \alpha^{-2} n^{2}$ of each other. As condition numbers are invariant under scaling, we may assume without loss of generality that they're in the range $\left[1,40 \kappa^{2} \alpha^{-2} n^{2}\right]$ by rescaling.

The assumption of $\mathcal{L}$ being strongly connected means $\mathbf{U}$ is connected. Therefore the minimum non-zero eigenvalue of $\mathbf{U}$ is at least $0.1 n^{-2}$, which occurs when $\mathbf{U}$ is a path, and the diagonal is either exactly equal to row / column sums, or larger by 1 on a single coordinate. The maximum on the other hand is bounded by $\operatorname{Trace}(\mathbf{U}) \leq 40 \kappa^{2} \alpha^{-2} n^{3}$, which gives that the condition number of $\mathbf{U}$ is bounded by $O\left(\kappa^{2} \alpha^{-2} n^{5}\right)$.

\section{A.2 Solving Eulerian Systems}

Lemma 57 implies that the condition number of $\mathbf{U}$ is polynomially bounded. Lemma 13 then implies that the condition number of $\mathbf{X}$ is also bounded by $O\left(\kappa^{2} \alpha^{-2} n^{5}\right)$. Therefore, the routines in this section are performing one-step preconditioning operations on polynomially conditioned matrices, and intuitively are stable. Formally, there are three main sources of numerical errors in this process:

1. The Woodbury matrix identity from Lemma 16.

2. Gaussian elimination involving the matrix $\mathbf{W}_{S}^{-1}+\mathbf{R} \widetilde{\mathbf{U}}^{+} \mathbf{R}^{\top}$. This matrix is polynomially conditioned, so LU/Cholesky factorizations on it are stable by assumption.

3. The calls to preconditioned iterative methods through Lemma 17. Since the relative condition number is bounded by $O\left(n^{2}\right)$, this is stable by stability results of Chebyshev iterations.

To show these bounds we need to bound the condition numbers of the input matrices.

Lemma 58. When Theorem 12 takes a matrix with condition number $\kappa$ and entries between $\left[U^{-1}, U\right]$ as input, all the intermediate matrices generated have condition number $\leq \kappa n^{10}$, and entries bounded by $\left[U^{-1} \kappa^{-1} n^{-10}, U \kappa n^{10}\right]$

Proof. These distortions happen in several places where we manipulate the matrices:

1. Lemma 15: here weights can be restricted to the range $[1, m]$ by lower bounding the probabilities at $1 / m$.

2. The Sherman-Morrison formula:

(a) $\mathbf{W}_{\mathbf{S}}$ has condition number $O\left(n^{2}\right)$ as above

(b) Entries of $\mathbf{U}^{+}$are pairwise effective resistances, and therefore bounded between $\left[U^{-1} n^{-2}, U n^{2}\right]$, so the matrix $\mathbf{R} \widetilde{\mathbf{U}}^{+} \mathbf{R}^{\top}$ is well conditioned, and have bounded entries.

(c) Compounding this with multiplying by $\mathbf{R} \widetilde{\mathbf{U}}^{+}$gives the bound on overall condition number and entry values

This allows us to control the overall accumulation of error through direct propagation of vector error bounds.

Lemma 59. If all the routines invoked in Theorem 12 are run to precision $U^{-1} \kappa^{-1} n^{-10}$, then the guarantees of Theorem 12 holds with $2 \epsilon$. 
Proof. The overall proof scheme is to view all numerical errors as perturbations with small $\ell_{2}$-norms after each step. Then the fact that the matrix are well conditioned means constant number of these steps can only increase error by a factor of $\operatorname{poly}(U, \kappa, n)$.

There are two main numerical routines invoked in the course of the algorithm.:

1. All the calls to $\mathbf{U}^{+}$are stable by the stability analyses of operator based solvers, since $\mathbf{U}$ is well-conditioned.

2. The calls to Chebyshev iteration invoked in Lemma17 has a condition number of $O\left(n^{2}\right)$, so all the operators are polynomially conditioned from the condition number bound on $\mathbf{Z}$. Therefore we can invoke known analyses of stability of Chebyshev iteration, such as Lemma 2.6.3. of [45].

Aside from that, the only other operations are matrix-vector multiplications.

\section{B Matrix Facts}

Lemma 60. Let $\mathbf{M} \in \mathcal{R}^{n \times n}$ be an invertible $R C D D$ Z-matrix. Then for any diagonal matrix $\mathbf{D} \in \mathcal{R}_{\geq 0}^{n \times n}$ and $\left\|(\mathbf{D}+\mathbf{M})^{-1}\right\|_{2} \leq\left\|\mathbf{M}^{-1}\right\|_{2}$.

Proof. For all $\alpha \in \mathcal{R}_{\geq 0}$ let $\mathbf{M}_{\alpha} \stackrel{\text { def }}{=} \alpha \mathbf{D}+\mathbf{M}$. Let $\mathbf{D}_{\alpha}=\operatorname{diag}(\alpha \mathbf{D}+\mathbf{M})$ and let $\mathbf{A}_{\alpha}=\operatorname{diag}(\mathbf{M})-\mathbf{M}$. Note that $\mathbf{M}_{\alpha}=\mathbf{D}-\mathbf{A}_{\alpha}$ for all $\alpha \geq 0$. Now since $\mathbf{M}$ is invertible and RCDD we know that $\mathbf{M}_{\alpha}$ is invertible and RCDD and

$$
\mathbf{M}_{\alpha}^{-1}=\left(\mathbf{D}_{\alpha}-\mathbf{A}\right)^{-1}=\mathbf{D}_{\alpha}^{-1}\left(\mathbf{I}-\mathbf{A D}_{\alpha}^{-1}\right)=\sum_{i=0}^{\infty} \mathbf{D}_{\alpha}^{-1}\left(\mathbf{A D}_{\alpha}^{-1}\right)^{i}
$$

Consequently, we see that $\mathbf{M}_{\alpha}^{-1}$ is a all positive matrix and $\left(\mathbf{M}_{\alpha}^{-1}\right)^{\top} \mathbf{M}_{\alpha}^{-1}$ is a all positive matrix both of which decrease entrywise as $\alpha$ increases. Since the maximum eigenvector of $\left(\mathbf{M}_{\alpha}^{-1}\right)^{\top} \mathbf{M}_{\alpha}^{-1}$ clearly has entries of all the same sign we see that $\left\|\mathbf{M}_{\alpha}^{-1}\right\|_{2}$ decreases monotonically as $\alpha$ increases. Considering $\alpha \in\{0,1\}$ yields the result.

Lemma 61. Let $\mathbf{M} \in \mathcal{R}^{n \times n}$ be either $C D D$ or $R D D$. Then $\|\mathbf{M} x\|_{1} \leq 2 \cdot \operatorname{tr}(\mathbf{M}) \cdot\|x\|_{\infty}$ for all $x \in \mathcal{R}^{n}$. Consequently, $\|\mathbf{M}\|_{2} \leq 2 \cdot \operatorname{tr}(\mathbf{M})$.

Proof. Since $\mathbf{M}$ is $\mathrm{CDD}$ or $\mathrm{RDD}$ we know $\mathbf{M}_{i i} \geq 0$ for all $i \in[n]$ and $\operatorname{tr}(\mathbf{M}) \geq \sum_{i \neq j \in[n]}\left|\mathbf{M}_{i j}\right|$. Consequently,

$$
\|\mathbf{M} x\|_{1} \leq \sum_{i, j \in[n]}\left|\mathbf{M}_{i j}\right| \cdot\|x\|_{\infty}=\left(\sum_{i \in[n]} \mathbf{M}_{i i}+\sum_{i \neq j \in[n]}\left|\mathbf{M}_{i j}\right|\right) \cdot\|x\|_{\infty} \leq 2 \cdot \operatorname{tr}(\mathbf{M}) \cdot\|x\|_{\infty} .
$$

Therefore, $\|\mathbf{M} x\|_{2} \leq\|\mathbf{M} x\|_{1} \leq 2 \cdot \operatorname{tr}(\mathbf{M}) \cdot\|x\|_{\infty} \leq 2 \cdot \operatorname{tr}(\mathbf{M}) \cdot\|x\|_{2}$.

Lemma 62. Let $\mathbf{M} \in \mathcal{R}^{n \times n}$ be an $\alpha$-CDD Z-matrix. For all $x \in \mathcal{R}_{\geq 0}^{n}$ we have $\operatorname{diag}(\mathbf{M})^{-1} x \leq$ $\mathbf{M}^{-1} x \leq \operatorname{diag}(\mathbf{M})^{-1} x+\frac{\|x\|_{1}}{\alpha} \operatorname{diag}(\mathbf{M})^{-1} 1$

Proof. Let $\mathbf{D} \stackrel{\text { def }}{=} \operatorname{diag}(\mathbf{M})$ and let $\mathbf{A} \stackrel{\text { def }}{=} \mathbf{D}-\mathbf{M}$ so $\mathbf{M}=\mathbf{D}-\mathbf{A}$. We see that

$$
\mathbf{M}^{-1}=(\mathbf{D}-\mathbf{A})^{-1}=\mathbf{D}^{-1}\left(\mathbf{I}-\mathbf{A D}^{-1}\right)=\sum_{i=0}^{\infty} \mathbf{D}^{-1}\left(\mathbf{A D}^{-1}\right)^{i}
$$


Consequently

$$
\mathbf{M}^{-1} x-\mathbf{D}^{-1} x=\mathbf{D}^{-1} \sum_{i=1}^{\infty}\left(\mathbf{A D}^{-1}\right)^{i} x
$$

Now since $\mathbf{M}$ is a $\alpha$-CDD Z-matrix we see that $\left(\mathbf{A D} \mathbf{D}^{-1}\right) x$ is a non-negative vector with $\left\|\mathbf{A D} \mathbf{D}^{-1} x\right\|_{1} \leq$ $\frac{1}{1+\alpha}\|x\|_{1}$. Consequently, $\sum_{i=1}^{\infty}\left(\mathbf{A D}^{-1}\right)^{i} x$ is a non-negative vector with $\ell_{1}$ norm at most

$$
\sum_{i=1}^{\infty}\left(\frac{1}{1+\alpha}\right)^{i}=\left(1-\frac{1}{1+\alpha}\right)^{-1}-1=\frac{1+\alpha}{\alpha}-1=\frac{1}{\alpha}
$$

Lemma 63. Let $\mathbf{M} \in \mathcal{R}^{n \times n}$, and let a diagonal matrix $\mathbf{D} \succeq 0$. Then $\left\|\left(\mathbf{M D}^{-1}\right)^{+}\right\|_{2} \leq\left\|\mathbf{M}^{+}\right\|_{2} \cdot\|\mathbf{D}\|_{2}$. Furthermore, $\kappa\left(\mathbf{M D}^{-1}\right)=\kappa\left(\left(\mathbf{M D}^{-1}\right)^{+}\right) \leq \kappa(\mathbf{M}) \kappa(\mathbf{D})$.

Proof. By definition, and using the fact that $\operatorname{ker}\left(\left(\mathbf{M} \mathbf{D}^{-1}\right)^{+}\right)=\operatorname{ker}\left(\mathbf{D}^{-1} \mathbf{M}^{\top}\right) \perp \operatorname{im}\left(\mathbf{M D}^{-1}\right)$, we have

$$
\begin{aligned}
\left\|\left(\mathbf{M D}^{-1}\right)^{+}\right\|_{2} & =\max _{x \perp \operatorname{ker}\left(\left(\mathbf{M D}^{-1}\right)^{+}\right), x \neq 0} \frac{\left\|\left(\mathbf{M D}^{-1}\right)^{+} x\right\|_{2}}{\|x\|_{2}}=\max _{y: \mathbf{M D}^{-1} y \neq 0} \frac{\left\|\left(\mathbf{M D}^{-1}\right)^{+} \mathbf{M D}^{-1} y\right\|_{2}}{\left\|\mathbf{M D}^{-1} y\right\|_{2}} \\
& =\max _{z: \mathbf{M} z \neq 0} \frac{\|\mathbf{D} z\|_{2}}{\|\mathbf{M} z\|_{2}}=\max _{z: \mathbf{M} z \neq 0}\left(\frac{\|z\|_{2}}{\|\mathbf{M} z\|_{2}} \cdot \frac{\|\mathbf{D} z\|_{2}}{\|z\|_{2}}\right) \\
& \leq \max _{z: \mathbf{M} z \neq 0} \frac{\|z\|_{2}}{\|\mathbf{M} z\|_{2}} \cdot \max _{z \neq 0} \frac{\|\mathbf{D} z\|_{2}}{\|z\|_{2}}=\left\|\mathbf{M}^{+}\right\|_{2} \cdot\|\mathbf{D}\|_{2} .
\end{aligned}
$$

Now we can plug in the definition for the condition number: $\kappa\left(\mathbf{M D}^{-1}\right)=\kappa\left(\left(\mathbf{M D}^{-1}\right)^{+}\right)=$ $\left\|\left(\mathbf{M D}^{-1}\right)^{+}\right\|_{2}\left\|\mathbf{M D}^{-1}\right\|_{2}$. For the first part we use the upper bound we just proved. For the second one, we bound it by $\left\|\mathbf{M D}^{-1}\right\|_{2} \leq\|\mathbf{M}\|_{2}\left\|\mathbf{D}^{-1}\right\|_{2}$. Combining these bounds we see that $\kappa\left(\mathbf{M D}^{-1}\right) \leq$ $\left\|\mathbf{M}^{+}\right\|_{2}\|\mathbf{D}\|_{2} \cdot\|\mathbf{M}\|_{2}\left\|\mathbf{D}^{-1}\right\|_{2}=\kappa(\mathbf{M}) \kappa(\mathbf{D})$.

Lemma 64. Let $\mathbf{M} \in \mathcal{R}^{n \times n}$ be an $\alpha-R C D D$ matrix with diagonal $\mathbf{D}$, let $\mathbf{U}_{\mathbf{M}}=\left(\mathbf{M}+\mathbf{M}^{\top}\right) / 2$, and let the $(n+1) \times n$ matrix $\mathbf{C}=\left(\begin{array}{c}\mathbf{I}_{n} \\ -\overrightarrow{1}_{n}^{\top}\end{array}\right)$. Then the $(n+1) \times(n+1)$ matrix $\mathbf{C U}_{\mathbf{M}} \mathbf{C}^{\top}$ satisfies $\kappa\left(\mathbf{C U}_{\mathbf{M}} \mathbf{C}^{\top}\right) \leq 2(1+\alpha) / \alpha \cdot n^{3} \cdot \kappa(\mathbf{D})$.

Proof. First, notice that if $\mathbf{M}$ is $\alpha$-RCDD, then it is also the case that $\mathbf{U}_{\mathbf{M}}$ is $\alpha$-RCDD. Then $\frac{\alpha}{1+\alpha} \mathbf{D} \preceq \mathbf{U}_{\mathbf{M}}$. Similarly, we have $\mathbf{U}_{\mathbf{M}} \preceq 2 \mathbf{D}$. Therefore $\kappa\left(\mathbf{C U}_{\mathbf{M}} \mathbf{C}^{\top}\right) \leq \kappa\left(\mathbf{C D C}^{\top}\right) \cdot 2(1+\alpha) / \alpha$. We see that $\mathbf{C D C}^{\top}$ is an undirected Laplacian with trace at most $2 \cdot \overrightarrow{1}^{\top} \mathbf{D} \overrightarrow{1} \leq 2 n \max _{i} \mathbf{D}_{i, i}$. Also, its minimum eigenvalue is therefore at least $\min _{i} \mathbf{D}_{i, i} \cdot n^{2}$. Hence $\kappa\left(\mathbf{C D C} \mathbf{C}^{\top}\right) \leq n^{3} \kappa(\mathbf{D})$, and our result follows.

Lemma 65. Let $\mathbf{A}$ and $\mathbf{A}^{\prime}=\mathbf{A}+\Delta \mathbf{A}$ be matrices with the same null space. Then, if $\|\Delta \mathbf{A}\|<$ $\left\|\mathbf{A}^{+}\right\|^{-1}$, then $\left\|(\mathbf{A}+\Delta \mathbf{A})^{+}\right\| \leq\left\|\mathbf{A}^{+}\right\| \cdot \frac{1}{1-\|\Delta \mathbf{A}\|\left\|\mathbf{A}^{+}\right\|}$.

Proof. Let $x$ be the largest singular vector of $\mathbf{A}^{\prime+}$, i.e. it is perpendicular to the kernel, and maximizes $\frac{\|x\|}{\left\|\mathbf{A}^{\prime} x\right\|}$. Then

$$
\frac{\|x\|}{\left\|\mathbf{A}^{\prime} x\right\|} \leq \frac{\|x\|}{\|\mathbf{A} x\|-\|\Delta \mathbf{A} x\| \mid}
$$


Since $\|\Delta \mathbf{A}\|<\left\|\mathbf{A}^{+}\right\|^{-1}$, we have that $\|\Delta \mathbf{A} x\| \leq\|\Delta \mathbf{A}\|\|x\|<\left\|\mathbf{A}^{+}\right\|^{-1} \cdot\|x\| \leq\|\mathbf{A} x\|$. Therefore we can drop the absolute value, and obtain

$$
\begin{aligned}
\frac{\|x\|}{\left\|\mathbf{A}^{\prime} x\right\|} & \leq \frac{\|x\|}{\|\mathbf{A} x\|-\|\Delta \mathbf{A} x\|}=\frac{\|x\|}{\|\mathbf{A} x\|} \cdot \frac{\|\mathbf{A} x\|}{\|\mathbf{A} x\|-\|\Delta \mathbf{A} x\|} \\
& =\frac{\|x\|}{\|\mathbf{A} x\|} \cdot \frac{1}{1-\|\Delta \mathbf{A} x\| /\|\mathbf{A} x\|} \leq \frac{\|x\|}{\|\mathbf{A} x\|} \cdot \frac{1}{1-\|\Delta \mathbf{A}\|\left\|\mathbf{A}^{+}\right\|} .
\end{aligned}
$$

Since $\frac{\|x\|}{\|\mathbf{A} x\|}$ is bounded by $\left\|\mathbf{A}^{+}\right\|$, our inequality follows.

Lemma 66. Let $\mathbf{A}$ and $\mathbf{A}^{\prime}=\mathbf{A}+\Delta \mathbf{A}$ be matrices with the same left and right null space, such that $\|\Delta \mathbf{A}\|<\left\|\mathbf{A}^{+}\right\|^{-1}$. Let $b$ be a vector in the image, and let $x=\mathbf{A}^{+} b$, and $\Delta x$ such that $x+\Delta x=(\mathbf{A}+\Delta \mathbf{A})^{+} b$. Then

$$
\frac{\|\Delta x\|}{\|x\|} \leq \frac{\left\|\mathbf{A}^{+}\right\|\|\Delta \mathbf{A}\|}{1-\left\|\mathbf{A}^{+}\right\|\|\Delta \mathbf{A}\|}
$$

Proof. Since $(\mathbf{A}+\Delta \mathbf{A})(x+\Delta x)=b$, we have $\Delta x=\mathbf{A}^{+}(-\Delta \mathbf{A} x-\Delta \mathbf{A} \Delta x)$. Applying triangle inequality we see that $\|\Delta x\| \leq\left\|\mathbf{A}^{+}\right\|\|\Delta \mathbf{A}\|(\|x\|+\|\Delta x\|)$. Therefore, after rearranging terms we obtain

$$
\|\Delta x\|\left(1-\left\|\mathbf{A}^{+}\right\|\|\Delta \mathbf{A}\|\right) \leq\|x\| \cdot\left\|\mathbf{A}^{+}\right\|\|\Delta \mathbf{A}\| .
$$

Finally, since $\left\|\mathbf{A}^{+}\right\|\|\Delta \mathbf{A}\|<1$, we get

$$
\frac{\|\Delta x\|}{\|x\|} \leq \frac{\left\|\mathbf{A}^{+}\right\|\|\Delta \mathbf{A}\|}{1-\left\|\mathbf{A}^{+}\right\|\|\Delta \mathbf{A}\|}
$$

\section{Deriving the Identities from Section 7.4}

\section{C.1 Formula for Hitting Times (Proof of Lemma 45)}

We derive the formula for $H_{u v}$ from first principles. Let $h^{v}$ be a vector where $h_{u}^{v}=H_{u v}$. Then, applying the definition we have that

$$
h_{u}^{v}=\left\{\begin{array}{ll}
0, & \text { if } u=v \\
1+\sum_{w} \mathbf{W}_{w u} \cdot h_{w}^{v}, & \text { if } u \neq v
\end{array}=\left\{\begin{array}{ll}
0, & \text { if } u=v \\
1+\left(\mathbf{W}^{\top} h^{v}\right)_{u}, & \text { if } u \neq v
\end{array} .\right.\right.
$$

Rearranging terms, we see that $h^{v}$ is a solution to the linear system

$$
\left[\begin{array}{cc}
{\left[\mathbf{I}-\mathbf{W}^{\top}\right.} & {\left[\mathbf{I}-\mathbf{W}^{\top}\right]_{B, v}} \\
\overrightarrow{0} & 1
\end{array}\right]\left[\begin{array}{c}
h_{B}^{v} \\
h_{v}^{v}
\end{array}\right]=\left[\begin{array}{l}
\overrightarrow{1} \\
0
\end{array}\right]
$$

where $B=V \backslash\{v\}$. Since every row is diagonally dominant, with the last row being strictly so, we see that a solution to this system of equation is unique. Consequently, it suffices to find a solution to this system of equations. Now, let $x$ be any solution to $(\mathbf{I}-\mathbf{W})^{\top} x=d$ where $d_{u}=1$ for $u \neq v$ and $d_{v}=1-\frac{1}{s_{v}}$. Since the graph associated to $\mathbf{W}$ is strongly connected, and $d \perp s$, there exists 
such an $x$ which is unique up to adding multiples of $\overrightarrow{1}$. Consequently, if we let $y=x-x_{v} \overrightarrow{1}$ then we see that $\left[\left(\mathbf{I}-\mathbf{W}^{\top}\right) y\right]_{u}=\left[\left(\mathbf{I}-\mathbf{W}^{\top}\right) x\right]_{u}=d_{u}=1$ for $u \neq v$, and $y_{v}=0$. Therefore, $y=h^{v}$ and

$$
H_{u v}=y_{u}=\overrightarrow{1}_{u}^{\top}\left(\mathbf{I}-\mathbf{W}^{\top}\right)^{+} d-\overrightarrow{1}_{v}^{\top}\left(\mathbf{I}-\mathbf{W}^{\top}\right)^{+} d=\left(\overrightarrow{1}_{u}-\overrightarrow{1}_{v}\right)^{\top}\left(\mathbf{I}-\mathbf{W}^{\top}\right)^{+} d .
$$

Plugging in $d=\overrightarrow{1}-\overrightarrow{1}_{v} \cdot \frac{1}{s_{v}}$, we obtain

$$
H_{u v}=\left(\overrightarrow{1}-\overrightarrow{1}_{v} \cdot \frac{1}{s_{v}}\right)^{\top}(\mathbf{I}-\mathbf{W})^{+}\left(\overrightarrow{1}_{u}-\overrightarrow{1}_{v}\right)
$$

\section{C.2 Formula for Commute Times (Proof of Lemma 50)}

Using the identity from Lemma 45, we can prove a nice formula for commute times.

$$
\begin{aligned}
C_{u v} & =H_{u v}+H_{v u} \\
& =\left(\overrightarrow{1}-\overrightarrow{1}_{v} \cdot \frac{1}{s_{v}}\right)^{\top}(\mathbf{I}-\mathbf{W})^{+}\left(\overrightarrow{1}_{u}-\overrightarrow{1}_{v}\right)+\left(\overrightarrow{1}-\overrightarrow{1}_{u} \cdot \frac{1}{s_{u}}\right)^{\top}(\mathbf{I}-\mathbf{W})^{+}\left(\overrightarrow{1}_{v}-\overrightarrow{1}_{u}\right) \\
& =\left(\overrightarrow{1}-\overrightarrow{1}_{v} \cdot \frac{1}{s_{v}}\right)^{\top}(\mathbf{I}-\mathbf{W})^{+}\left(\overrightarrow{1}_{u}-\overrightarrow{1}_{v}\right)-\left(\overrightarrow{1}-\overrightarrow{1}_{u} \cdot \frac{1}{s_{u}}\right)^{\top}(\mathbf{I}-\mathbf{W})^{+}\left(\overrightarrow{1}_{u}-\overrightarrow{1}_{v}\right) \\
& =\left(\overrightarrow{1}_{u} \cdot \frac{1}{s_{u}}-\overrightarrow{1}_{v} \cdot \frac{1}{s_{v}}\right)^{\top}(\mathbf{I}-\mathbf{W})^{+}\left(\overrightarrow{1}_{u}-\overrightarrow{1}_{v}\right) \\
& =\left(\overrightarrow{1}_{u}-\overrightarrow{1}_{v}\right)^{\top} \mathbf{S}^{-1}(\mathbf{I}-\mathbf{W})^{+}\left(\overrightarrow{1}_{u}-\overrightarrow{1}_{v}\right) .
\end{aligned}
$$

Furthermore, this also gives

$$
C_{u v}=\left(\overrightarrow{1}_{u}-\overrightarrow{1}_{v}\right)((\mathbf{I}-\mathbf{W}) \mathbf{S})^{+}\left(\overrightarrow{1}_{u}-\overrightarrow{1}_{v}\right) .
$$

While in general $((\mathbf{I}-\mathbf{W}) \mathbf{S})^{+} \neq \mathbf{S}^{-1}(\mathbf{I}-\mathbf{W})^{+}$, in this case we notice that

$$
\left(\overrightarrow{1}_{u}-\overrightarrow{1}_{v}\right)^{\top}\left(\mathbf{S}^{-1}(\mathbf{I}-\mathbf{W})^{+}-((\mathbf{I}-\mathbf{W}) \mathbf{S})^{+}\right)\left(\overrightarrow{1}_{u}-\overrightarrow{1}_{v}\right)=0,
$$

and the result follows.

Indeed, we can immediately see that $\left((\mathbf{I}-\mathbf{W})^{+}-\mathbf{S}((\mathbf{I}-\mathbf{W}) \mathbf{S})^{+}\right)\left(\overrightarrow{1}_{u}-\overrightarrow{1}_{v}\right) \in \operatorname{ker}(\mathbf{I}-\mathbf{W})$ : using the fact that $\overrightarrow{1}_{u}-\overrightarrow{1}_{v} \perp \operatorname{ker}\left(\mathbf{I}-\mathbf{W}^{\top}\right)$, so $\overrightarrow{1}_{u}-\overrightarrow{1}_{v} \in \operatorname{Im}(\mathbf{I}-\mathbf{W})$, we obtain $(\mathbf{I}-$ $\mathbf{W})\left((\mathbf{I}-\mathbf{W})^{+}-\mathbf{S}((\mathbf{I}-\mathbf{W}) \mathbf{S})^{+}\right)\left(\overrightarrow{1}_{u}-\overrightarrow{1}_{v}\right)=\left(\mathbf{I}_{I m(\mathbf{I}-\mathbf{W})}-\mathbf{I}_{I m((\mathbf{I}-\mathbf{W}) \mathbf{S})}\right)\left(\overrightarrow{1}_{u}-\overrightarrow{1}_{v}\right)=0$. Therefore, $\left(\overrightarrow{1}_{u}-\overrightarrow{1}_{v}\right)^{\top}\left(\mathbf{S}^{-1}(\mathbf{I}-\mathbf{W})^{+}-((\mathbf{I}-\mathbf{W}) \mathbf{S})^{+}\right)\left(\overrightarrow{1}_{u}-\overrightarrow{1}_{v}\right)=\left(\overrightarrow{1}_{u}-\overrightarrow{1}_{v}\right)^{\top} \mathbf{S}^{-1} \cdot k$ where $k \in \operatorname{ker}(\mathbf{I}-\mathbf{W})$. Hence, writing $k=\alpha s$ for some constant $\alpha$, we have $\left(\overrightarrow{1}_{u}-\overrightarrow{1}_{v}\right)^{\top} \mathbf{S}^{-1} k=\left(\overrightarrow{1}_{u}-\overrightarrow{1}_{v}\right)^{\top} \mathbf{S}^{-1} \cdot \alpha s=\left(\overrightarrow{1}_{u}-\overrightarrow{1}_{v}\right)^{\top} \cdot \alpha \overrightarrow{1}=0$, which yields the desired result.

Finally, for the third identity, since the vectors on both sides are symmetric, we can write

$$
C_{u v}=\left(\overrightarrow{1}_{u}-\overrightarrow{1}_{v}\right)^{\top} \mathcal{L}_{b}^{+}\left(\overrightarrow{1}_{u}-\overrightarrow{1}_{v}\right)=\frac{1}{2}\left(\overrightarrow{1}_{u}-\overrightarrow{1}_{v}\right)^{\top}\left(\mathcal{L}_{b}^{+}+\left(\mathcal{L}_{b}^{+}\right)^{\top}\right)\left(\overrightarrow{1}_{u}-\overrightarrow{1}_{v}\right) .
$$

We will show that the matrix that appears in the middle is, as a matter of fact, symmetric positive semi-definite. In order to do so, we first use the fact that

$$
\mathcal{L}_{b}^{+}+\left(\mathcal{L}_{b}^{+}\right)^{\top}=\mathcal{L}_{b}^{+}\left(\mathcal{L}_{b}+\mathcal{L}_{b}^{\top}\right)\left(\mathcal{L}_{b}^{+}\right)^{\top}
$$

which follows immediately by expanding the right hand side. This identity critically uses the fact that $\mathcal{L}_{b}$ is Eulerian, and therefore the right and left null spaces coincide. Plugging this into the previous formula for commute times yields our desired identity. 


\section{C.3 Formula for Escape Probabilities (Proof of Lemma 48)}

Just like we did for hitting times, we can derive the formula for escape probabilities from first principles. In particular, the definition gives us

$$
p_{i}=\left\{\begin{array}{ll}
1, & \text { if } i=u \\
0, & \text { if } i=v \\
\sum_{j} \mathbf{W}_{i j} \cdot p_{j}, & \text { otherwise }
\end{array}=\left\{\begin{array}{ll}
1, & \text { if } i=u \\
0, & \text { if } i=v \\
\mathbf{W} \cdot p, & \text { otherwise }
\end{array} .\right.\right.
$$

Equivalently, this yields the linear system:

$$
\left[\begin{array}{ccc}
{[\mathbf{I}-\mathbf{W}]_{B, B}} & {[\mathbf{I}-\mathbf{W}]_{B, s}} & {[\mathbf{I}-\mathbf{W}]_{B, t}} \\
0 & 1 & 0 \\
0 & 0 & 1
\end{array}\right]\left[\begin{array}{c}
p_{B} \\
p_{u} \\
p_{v}
\end{array}\right]=\left[\begin{array}{c}
\overrightarrow{0} \\
1 \\
0
\end{array}\right],
$$

where $B=V \backslash\{u, v\}$. Again, since every row is diagonally dominant, and the last two are strictly so, we see that a solution to this system is unique. So we only need to find a solution to this system.

Now let $x$ be a solution to $(\mathbf{I}-\mathbf{W}) x=\overrightarrow{1}_{u}-\overrightarrow{1}_{v}$. Since $\overrightarrow{1}_{u}-\overrightarrow{1}_{v} \perp \overrightarrow{1}$, the solution exists. Also, since the graph associated to $\mathbf{W}$ is strongly connected, it is unique up to adding multiples of the stationary. Therefore letting $y=x-s \cdot \frac{x_{v}}{s_{v}}$ all the constraints of the system are satisfied, except $y_{u}=1$. Setting $y^{\prime}=\frac{1}{y_{u}} \cdot y=\left(x_{u}-s_{u} \cdot \frac{x_{v}}{s_{v}}\right)^{-1}\left(x-s \cdot \frac{x_{v}}{s_{v}}\right)=\left(s_{u}\left(\frac{x_{u}}{s_{u}}-\frac{x_{v}}{s_{v}}\right)\right)^{-1} \cdot\left(x-s \cdot \frac{x_{v}}{s_{v}}\right)$ yields a solution to the system. Consequently, $p=y^{\prime}$ and the result follows.

Next, we bound the coefficients.

We have $|\alpha|=\left|\frac{x_{v}}{s_{v}}\right| \leq \frac{1}{s_{\min }} \cdot\left\|(\mathbf{I}-\mathbf{W})^{+}\left(\overrightarrow{1}_{u}-\overrightarrow{1}_{v}\right)\right\|_{\infty} \leq \frac{1}{s_{\min }} \cdot\left\|(\mathbf{I}-\mathbf{W})^{+}\left(\overrightarrow{1}_{u}-\overrightarrow{1}_{v}\right)\right\|_{\infty} \leq \frac{1}{s_{\min }}$. $\left\|(\mathbf{I}-\mathbf{W})^{+}\left(\overrightarrow{1}_{u}-\overrightarrow{1}_{v}\right)\right\|_{1} \leq \frac{1}{s_{\min }} \cdot\left\|(\mathbf{I}-\mathbf{W})^{+}\right\|_{1}\left\|\overrightarrow{1}_{u}-\overrightarrow{1}_{v}\right\|_{1}$. Plugging in Theorem 21, we obtain that $|\alpha| \leq \frac{t_{\operatorname{mix}}}{s_{\min }} \cdot 8 \sqrt{n} \log _{2} n$.

Also, $\beta=\frac{1}{s_{u}\left(\frac{x_{u}}{s_{u}}-\frac{x_{v}}{s_{v}}\right)}=\frac{1}{s_{u}\left(\overrightarrow{1}_{u}-\overrightarrow{1}_{v}\right)^{\top} \mathbf{S}^{-1}(\mathbf{I}-\mathbf{W})^{+}\left(\overrightarrow{1}_{u}-\overrightarrow{1}_{v}\right)}$. As seen from Lemma 50, the term $\left(\overrightarrow{1}_{u}-\right.$ $\left.\overrightarrow{1_{v}}\right)^{\top} \mathbf{S}^{-1}(\mathbf{I}-\mathbf{W})^{+}\left(\overrightarrow{1}_{u}-\overrightarrow{1}_{v}\right)$ is precisely the commute time $C_{u v}$ which satisfies $1 \leq C_{u v}$ by definition. Also, we have that $C_{u v}=\left(\overrightarrow{1}_{u}-\overrightarrow{1}_{v}\right)^{\top} \mathbf{S}^{-1}(\mathbf{I}-\mathbf{W})^{+}\left(\overrightarrow{1}_{u}-\overrightarrow{1}_{v}\right) \leq\left\|\mathbf{S}^{-1}\left(\overrightarrow{1}_{u}-\overrightarrow{1}_{v}\right)\right\|_{\infty} \cdot\left\|(\mathbf{I}-\mathbf{W})^{+}\left(\overrightarrow{1}_{u}-\overrightarrow{1}_{v}\right)\right\|_{1} \leq$ $\frac{1}{s_{\min }} \cdot\left\|(\mathbf{I}-\mathbf{W})^{+}\left(\overrightarrow{1}_{u}-\overrightarrow{1}_{v}\right)\right\|_{1} \leq \frac{t_{\text {mix }}}{s_{\min }} \cdot 8 \sqrt{n} \log _{2} n$. Therefore $\frac{1}{t_{\operatorname{mix}} \cdot 8 \sqrt{n} \log _{2} n} \leq \beta \leq \frac{1}{s_{\min }}$. 